\title{
OPERATOR MANIFOLD APPROACH TO GEOMETRY AND PARTICLE PHYSICS
}

\author{
G.T.Ter-Kazariant \\ Byurakan Astrophysical Observatory, Armenia 378433 \\ June 3, 1996
}

\begin{abstract}
The question that guides our discussion is how did the geometry and particles come into being? To explore this query the present theory reveals primordial deeper structures underlying fundamental concepts of contemporary physics. We begin with a drastic revision of a role of local internal symmetries in physical concept of curved geometry. Under the reflection of fields and their dynamics from Minkowski space to Riemannian a standard gauge principle of local internal symmetries was generalized. The gravitation gauge group is proposed, which was generated by hidden local internal symmetries. In all circumstances, it seemed to be of the most importance for understanding of physical nature of gravity. Last two parts of this paper address to the question of physical origin of geometry and basic concepts of particle physics such as the fundamental fields of quarks with the spins and various quantum numbers, internal symmetries and so forth; also four basic principles of Relativity, Quantum, Gauge and Color Confinement, which are, as it was proven, all derivative and come into being simultaneously. The substance out of which the geometry and particles are made is a set of new physical structures - the goyaks $\mathrm{E}$, which are involved into reciprocal linkage establishing processes. The most promising aspect of our approach so far is the fact that many of the important anticipated properties, basic concepts and principles of particle physics are appeared quite naturally in the framework of suggested theory.
\end{abstract}

\section{Introduction}

Although a definite pattern for the theoretical description of particle physics has emerged, which is attractive enough both theoretically and phenomenologically, but it could not be regarded as the final word in particle physics and many fundamental questions have yet to be answered. In all previous studies the concepts and right symmetries, also basic principles of particle physics have been put into theory by hand. The difficulties associated with this step are notorious. In view of all this, we are led to consider the problem, as it was seen at the outset, how to find out the substance or basic structures, something deeper, that underly both geometry and particles? It will be appropriate to turn to them as the primordial deeper structures. The absence of the vital physical theory, which will be able to reveal these structures and answer to the right questions mentioned above, imperatively stimulates the search for general constructive principles. The guiding line framing our discussion throughout this paper is the generalization and further expansion of ideas of the theory of distortion of space-time continuum [1-5], wherein, as it is believed, the space-time is not pre-determined background on which physical processes take place, but

\footnotetext{
${ }^{1}$ E-mail address:gago@bao.sci.am

${ }^{2}$ goyak in Armenian means an existence (an existing structure). This term has been used in [1-5]
} 
a dynamical entity itself. We start with the formulation of general gauge principle, with in the scope of which we outlined the rules governing the general distortion of geometry. The last two parts of the theory are a revised and brief version of $[4,5]$. In second part our task is to develop and understand the conceptual foundations for our viewpoint. We elaborate a mathematical framework in order to describe the persistent processes of creation and annihilation of regular goyaks. It is, in fact, a still wider generalization of the familiar methods of secondary quantization with appropriate expansion over the geometric objects. Hence, it gives rise to the formalism of operator manifold, which yields the quantization of geometry, which differs in principle from all earlier studies. The geometry realization condition should be cleared up. But it turned out to be a trivial one, which leads to geometry without particles and interactions. There is still an other choice of realization of geometry, which subsequently yields the geometry with the particles and interactions. Connected to it, we employ the wave functions of distorted goyaks to extend the knowledge here gained regarding the quark fields being introduced in the color space of internal degrees of freedom. The local distortion rotations around each fixed axis yield the quarks or anti-quarks. They obey exact color confinement, and the spectrum of hadrons would emerge as the spectrum of the color singlet states. Finally, it has been shown that the gauge principle holds for any physical system which can be treated as a definite system of distorted goyaks. The theory of goyaks predicts a class of possible models of internal symmetries, which utilize the idea of gauge symmetry and reproduce the known phenomenology of electromagnetic, weak and strong interactions. To save writing we guess it worthwhile to leave the other concepts such as the flavors and so forth with associated aspects of particle physics for an other treatment. It will not concern us here and must be further discussed. Surely this is an important subject for separate research.

\section{Part I. General Gauge Principle and Gravitation}

\section{Formulation of Principle}

In spite of unrivaled by its simplicity and beautiful features, Einstein 's classical theory of gravitation clashes with some basic principle of field theory. This state of affairs has not much changed up to present and proposed abundant models of gravitation are not conductive to provide unartificial and unique recipe for resolving controversial problems of energy-momentum conservation laws of gravitational interacting fields, localization of energy of gravitation waves and also severe problems involved in quantum gravity. It may seem foolhardy to think the rules governing such issues in scope of the theory accounting for gravitation entirely in terms of intricated Riemannian geometry. The difficulties associated with this step are notorious, however, these difficulties are technical. In the main, they stem from the fact that Riemannian geometry, in general, has not admitted a group of isometries. So, the Poincare transformations no longer act as isometries. For example, it is not possible to define energy -momentum as Noether currents related to exact symmetries and so on. On the other hand, the concepts of local internal symmetries and gauge fields [6-8] have became a powerful tool and successfully utilized for the study of electroweak and strong interactions. The intensive attempts have been made for constructing a gauge theory of gravitation. But they are complicated sure enough and 
not generally acceptable. Since none of the solutions of the problems of determination of the gauge group of gravitation and Lagrangian of gravitational field proposed up to the present seems to be wholly convincing, one might gain insight into some of unknown features of phenomena of local internal symmetries and gravity by investigating a new approaches in hope of resolving such issues.. Effecting a reconciliation it is our purpose to explore a number of fascinating features of generating the gauge group of gravitation by hidden local internal symmetry. In line with this we feel it of the most importance to generalize standard gauge principle of local internal symmetries. While, a second trend emerged as a formalism of the reflection of physical fields and their dynamics from Minkowski space to Riemannian. There is an attempt to supply some of the answers in a concise form to the crucial problems of gravitation and to trace some of the major currents of thoughts under a novel view-point. Exploiting the whole advantages of the field theory in terms of flat space, a particular emphasis will be placed just on the formalism of the reflection. The energy -momentum conservation laws of gravitational interacting fields are well -defined by exploiting whole advantages of auxiliary shadow fields on flat shadow space. The developed mechanism enables one to infer Einstein 's equation of gravitation, but only with strong difference from Einstein 's theory at the vital point of well -defined energy -momentum tensor of gravitational field and conservation laws. The curvature as well as general distortion of geometry at hidden group $U^{l o c}(1)$ are considered. This is not a final report on a closed subject, but it is hoped that suggested theory will serve as useful introduction and that it will thereby add the knowledge on the role of local internal symmetries in physical concept of curved geometry.

In standard picture, suppose a massless gauge field $\mathbf{B}_{l}\left(x_{f}\right)=T^{a} B_{l}^{a}\left(x_{f}\right)$ with the values in Lie algebra of group $\mathrm{G}$ is a local form of expression of connection in principle bundle with a structure group $\mathrm{G}$. Collection of matter fields are defined as the sections of vector bundles associated with $\mathrm{G}$ by reflection $\Phi_{f}: M^{4} \rightarrow E$ that $p \Phi_{f}\left(x_{f}\right)=x_{f}$, where $x_{f} \in M^{4}$ is a space-time point of Minkowski flat space specified by index $\left({ }_{f}\right)$. The $\Phi_{f}$ is a column vector denoting particular component of field taking values in standard fiber $F_{x_{f}}$ upon $x_{f}$ : $p^{-1}\left(U^{(f)}\right)=U^{(f)} \otimes F_{x_{f}}$, where $U^{(f)}$ is a region of base of principle bundle upon which an expansion into direct product $p^{-1}\left(U^{(f)}\right)=U^{(f)} \otimes G$ is defined. The various suffixes of $\Phi_{f}$ are left implicit. The fiber is Hilbert vector space on which a linear representation $U_{f}\left(x_{f}\right)=\exp \left(-T^{a} \theta_{f}^{a}\left(x_{f}\right)\right)$ of group $\mathrm{G}$ with structure constants $C^{a b c}$ is given. This space regarded as Lie algebra of group $\mathrm{G}$ upon which Lie algebra acts according to law of adjoint representation: $\mathbf{B} \leftrightarrow \operatorname{ad} \mathbf{B} \Phi_{f} \rightarrow\left[\mathbf{B}, \Phi_{f}\right]$. To facilitate writing, we shall consider, in the main, the most important fields of spin $0, \frac{1}{2}, 1$. But developed method may be readily extended to the field of arbitrary spin $s$, since latter will be treated as a system of $2 s$ fermions of half -integral spin. In order to generalize standard gauge principle below we proceed with preliminary discussion.

a. As a starting point we shall assume that under gauge field $\mathbf{B}_{f}$ the basis vectors $e_{f}$ transformed into four vector fields

$$
\hat{e}^{\mu}\left(\kappa \mathbf{B}_{f}\right)=\hat{D}_{l}^{\mu}\left(\kappa \mathbf{B}_{f}\right) e_{f}^{l}
$$

where $\hat{D}_{l}^{\mu}$ are real -valued matrix -functions of $\mathbf{B}_{f}, \kappa$ is universal coupling constant by which gravitational constant will be expressed (see eq.(4.7)). The double occurrence of dummy indices, as usual, will be taken to denote a summation extended over their all values. Explicit form of $\hat{D}_{l}^{\mu}$ will not concern us here, which must be defined under 
concrete physical considerations [1-5] (sec. 5,6). However, two of the most common restrictions will be placed upon these functions. As far as all known interactions are studied on the base of commutative geometry, then, first of all, the functions $\hat{D}_{l}^{\mu}$ will be diagonalized at given $(\mu, l)$. As a real -valued function of Hermitian matrix $\mathbf{B}_{f}$, each of them is Hermitian too and may be diagonalized by proper unitary matrix. Hence $D_{l}^{\mu}\left(\kappa \mathbf{B}_{f}\right)=\operatorname{diag}\left(\lambda_{l 1}^{\mu}, \lambda_{l 2}^{\mu}, \lambda_{l 3}^{\mu}, \lambda_{l 4}^{\mu}\right)$, provided with eigen -values $\lambda_{l i}^{\mu}$ as the roots of polynomial characteristic equation

$$
C\left(\lambda_{l}^{\mu}\right)=\operatorname{det}\left(\lambda_{l}^{\mu} I-\hat{D}_{l}^{\mu}\right)=0
$$

Thus

$$
e^{\mu}\left(\kappa \mathbf{B}_{f}\right)=D_{l}^{\mu}\left(\kappa \mathbf{B}_{f}\right) e_{f}^{l}
$$

lead to commutative geometry. Consequently, if $e_{\nu}\left(\kappa \mathbf{B}_{f}\right)$ denotes the inverse matrix vector $\|D\| \neq 0$, then $<e^{\mu}, e_{\nu}>=D_{l}^{\mu} D_{\nu}^{l}=\delta_{\nu}^{\mu}, \quad D_{l}^{\mu} D_{\mu}^{k}=\delta_{l}^{k}$. A bilinear form on vector fields of sections $\tau$ of tangent bundle of $R^{4}: \quad \hat{g}: \tau \otimes \tau \rightarrow C^{\infty}\left(R^{4}\right)$, namely the metric $\left(g_{\mu \nu}\right)$, is a section of conjugate vector bundle $S^{2} \tau^{*}$ (symmetric part of tensor degree) with corresponding components in basis $e_{\mu}\left(\kappa \mathbf{B}_{f}\right)$

$$
g_{\mu \nu}=<e_{\mu}, e_{\nu}>=<e_{\nu}, e_{\mu}>=g_{\nu \mu}, \quad e_{\mu}=g_{\mu \nu} e^{\nu}
$$

In holonomic basis $\hat{g}=g_{\mu \nu} d x^{\mu} \otimes d x^{\nu}$. One now has to impose a second restriction on the functions $D_{l}^{\mu}$ by placing stringent condition upon the tensor $\omega_{l}^{m}\left(x_{f}\right)$ implying

$$
\partial_{l}^{f} D_{k}^{\mu}\left(\kappa \mathbf{B}_{f}\right)=\partial_{k}^{f}\left(\omega_{l}^{m}\left(x_{f}\right) D_{m}^{\mu}\left(\kappa \mathbf{B}_{f}\right)\right) \text {, }
$$

where $\partial_{l}^{f}=\frac{\partial}{\partial x_{f}^{l}}$, that the $\omega=\omega_{l}^{l}$ should be Lorentz scalar function of the trace of curvature form $\Omega$ of connection $\mathbf{B}_{l}$ with the values in Lie algebra of group $G$. That is

$$
\omega=\omega(\operatorname{tr} \Omega)=\omega(d \operatorname{tr} \mathbf{B}),
$$

provided

$$
\begin{aligned}
& \Omega=\sum_{l<k} \mathbf{F}_{l k}^{f} d x_{f}^{l} \wedge d x_{f}^{k}, \quad \mathbf{B}=\mathbf{B}_{l}^{f} d x_{f}^{l}, \\
& \mathbf{F}_{l k}^{f}=\partial_{l}^{f} \mathbf{B}_{k}-\partial_{k}^{f} \mathbf{B}_{l}-i g\left[\mathbf{B}_{l}, \mathbf{B}_{k}\right] .
\end{aligned}
$$

There up on a functional $\omega$ has a null variational derivative $\frac{\delta \omega(\operatorname{tr} \Omega)}{\delta \mathbf{B}_{l}}=0$ at local variations of connection $\mathbf{B}_{l} \rightarrow \mathbf{B}_{l}+\delta \mathbf{B}_{l}$, namely, $\omega$ is invariant under Lorentz $(\Lambda)$ and G-gauge groups $\omega=i n v(\Lambda, G)$.

b. Constructing a diffeomorphism $x^{\mu}\left(x_{f}^{l}\right): M^{4} \rightarrow R^{4}$, the holonomic functions $x^{\mu}\left(x_{f}^{l}\right)$ satisfy defining relation

$$
e_{\mu} \psi_{l}^{\mu}=e_{l}^{f}+\chi_{l}^{f}\left(\mathbf{B}_{f}\right)
$$

where

$$
\chi_{l}^{f}\left(\mathbf{B}_{f}\right)=e_{\mu} \chi_{l}^{\mu}=-\frac{1}{2} e_{\mu} \int_{0}^{x_{f}}\left(\partial_{k}^{f} D_{l}^{\mu}-\partial_{l}^{f} D_{k}^{\mu}\right) d x_{f}^{k} .
$$

The following notational conventions will be used throughout: $\psi_{l}^{\mu}=\partial_{l}^{f} x^{\mu}$, where the indices $\mu, \nu, \lambda, \tau, \sigma, \kappa$ stand for variables in $R^{4}$, when $l, k, m, n, i, j$ refer to $M^{4}$. A closer 
examination of eq. (2.9) shows that covector $\chi_{l}^{f}\left(\mathbf{B}_{f}\right)$ realizes coordinates $x^{\mu}$ by providing a criteria of integration

$$
\partial_{k}^{f} \psi_{l}^{\mu}=\partial_{l}^{f} \psi_{k}^{\mu}
$$

and undegeneration $\|\psi\| \neq 0[9,10]$. Due to eq.(2.6) and eq.(2.9), one has Lorentz scalar gauge invariant functions

$$
\chi=<e_{f}^{l}, \chi_{l}^{f}>=\frac{\omega}{2}-2, \quad S=\frac{1}{4} \psi_{\mu}^{l} D_{l}^{\mu}=\frac{1}{2}\left(1+\frac{\omega}{4}\right) .
$$

So, out of a set of arbitrary curvilinear coordinates in $R^{4}$ the real-curvilinear coordinates may be distinguished, which satisfy eq.(2.8) under all possible Lorentz and gauge transformations. There is a single -valued conformity between corresponding tensors with various suffixes on $R^{4}$ and $M^{4}$. While, each co- or contra -variant index transformed incorporating respectively with functions $\psi_{l}^{\mu}$ or $\psi_{\mu}^{l}$. Each transformation of real -curvilinear coordinates $x^{\prime \mu^{\prime}} \rightarrow x^{\mu}$ was generated by some Lorentz and gauge transformations

$$
\frac{\partial x^{\mu^{\prime}}}{\partial x^{\mu}}=\psi_{l^{\prime}}^{\mu^{\prime}}\left(B_{f}^{\prime}\right) \psi_{\mu}^{l}\left(B_{f}\right) \Lambda_{l}^{l^{\prime}}
$$

There would then exist preferred systems and group of transformations of real -curvilinear coordinates in $R^{4}$. The wider group of transformations of arbitrary curvilinear coordinates in $R^{4}$ would then be of no importance for the field dynamics. If an inverse function $\psi_{\mu}^{l}$ meets condition

$$
\frac{\partial \psi_{\mu}^{l}}{d x^{\nu}} \neq \Gamma_{\mu \nu}^{\lambda} \psi_{\lambda}^{l}
$$

where $\Gamma_{\mu \nu}^{\lambda}$ is the usual Christoffel symbol agreed with a metric $g_{\mu \nu}$, then a curvature of $R^{4}$ is not vanished $[11,12]$.

In pursuing the original problem further we are led to the principle point of drastic change of standard gauge scheme to assume hereafter that single-valued, smooth, double-sided reflection of fields $\Phi_{f} \rightarrow \Phi\left(\Phi_{f}\right): F_{x_{f}} \rightarrow F_{x}$, namely $\Phi_{f}^{l \cdots m}\left(x_{f}\right)$, takes place under local group $G$, where $\Phi_{f} \subset F_{x_{f}}, \Phi \subset F_{x}, F_{x}$ is the fiber upon $x: p^{-1}(U)=U \otimes F_{x}$, $U$ is the region of base $R^{4}$. The tensor suffixes were only put forth in illustration of a point at issue. Then

$$
\Phi^{\mu \cdots \delta}(x)=\psi_{l}^{\mu} \cdots \psi_{m}^{\delta} R\left(\mathbf{B}_{f}\right) \Phi_{f}^{l \cdots m}\left(x_{f}\right) \equiv\left(R_{\psi}\right)_{l \cdots m}^{\mu \cdots \delta} \Phi_{f}^{l \cdots m}\left(x_{f}\right)
$$

where $R\left(\mathbf{B}_{f}\right)$ is a reflection matrix. The idea of general gauge principle may be framed into requirement of invariance of physical system of fields $\Phi(x)$ under the finite local gauge transformations $U_{R}=R_{\psi}^{\prime} U_{f} R_{\psi}^{+}$of the Lie group of gravitation $G_{R}$ generated by $G$, where $R_{\psi}^{\prime}=R_{\psi}\left(\mathbf{B}_{f}^{\prime}\right)$ if gauge field $\mathbf{B}_{f}\left(x_{f}\right)$ was transformed under $G$ in standard form. While the corresponding transformations of fields $\Phi(x)$ and their covariant derivatives are written

$$
\begin{aligned}
& \Phi^{\prime}(x)=U_{R}(x) \Phi(x), \\
& \left(g^{\mu}(x) \nabla_{\mu} \Phi(x)\right)^{\prime}=U_{R}(x)\left(g^{\mu}(x) \nabla_{\mu} \Phi(x)\right) .
\end{aligned}
$$

The solution of eq.(2.15) may be readily obtained as the reflection of covariant derivatives

$$
g^{\nu}(x) \nabla_{\nu} \Phi^{\mu \cdots \delta}(x)=S\left(B_{f}\right) \psi_{l}^{\mu} \cdots \psi_{m}^{\delta} R\left(\mathbf{B}_{f}\right) \gamma^{k} D_{k} \Phi_{f}^{l \cdots m}\left(x_{f}\right)
$$


where $D_{l}=\partial_{l}^{f}-i g \mathbf{B}_{l}\left(x_{f}\right), S\left(B_{f}\right)$ is gauge invariant Lorentz scalar, $\nabla_{\mu}$ is covariant derivative in $R^{4}: \nabla_{\mu}=\partial_{\mu}+\Gamma_{\mu}$, provided with connection [11] $\Gamma_{\mu}(x)=\frac{1}{2} \Sigma^{\alpha \beta} V_{\alpha}^{\nu}(x) \partial_{\mu} V_{\beta \nu}(x)$, the $\Sigma^{\alpha \beta}$ are generators of Lorentz group, $V_{\alpha}^{\mu}(x)$ are the components of affine tetrad vectors $e^{\alpha}$ in used coordinate net $x^{\mu}: V_{\alpha}^{\mu}(x)=<e^{\mu}, e_{\alpha}>$; one has $g^{\mu}(x) \Rightarrow e^{\mu}(x)$ and $\gamma^{l} \Rightarrow e_{f}^{l}$ for fields $(s=0,1)$; but $g^{\mu}(x)=V_{\alpha}^{\mu}(x) \gamma^{\alpha}$ for spinor field $\left(s=\frac{1}{2}\right)$, where $\gamma^{\alpha}$ are Dirac's matrices. Since the fields $\Phi_{f}\left(x_{f}\right)$ no longer hold, the reflected ones $\Phi(x)$ will be regarded as the real physical fields. But a conformity eq.(2.14) and eq $(2.16)$ enables $\Phi_{f}\left(x_{f}\right)$ to serve as an auxiliary shadow fields on shadow flat space $M^{4}$. These notions arise basically from the most important fact that a Lagrangian $L(x)$ of fields $\Phi(x)$ may be obtained under the reflection from a Lagrangian $L_{f}\left(x_{f}\right)$ of corresponding shadow fields and vice versa. Certainly, the $L(x)$ is also an invariant under the wider group of arbitrary curvilinear transformations $x \rightarrow x^{\prime}$ in $R^{4}$

$$
\left.J_{\psi} L(x)\right|_{i n v\left(G_{R} ; x \rightarrow x^{\prime}\right)}=\left.L_{f}\left(x_{f}\right)\right|_{i n v(\Lambda ; G)},
$$

where $J_{\psi}=\|\psi\| \sqrt{ }=\left(1+2\left\|<e_{l}^{f}, \chi_{k}^{f}>\right\|+\left\|<\chi_{l}^{f}, \chi_{k}^{f}>\right\|\right)^{1 / 2}$ (see eq.(4.1)). While, the internal gauge symmetry $G$ remained hidden symmetry, since it screened by gauge group of gravitation $G_{R}$. At last, one notes that the tetrad $e^{\alpha}$ and basis $e_{f}^{l}$ vectors meet a condition

$$
\begin{aligned}
& \rho_{l}^{\alpha}\left(x, x_{f}\right)=<e^{\alpha}, e_{l}^{f}>=V_{\mu}^{\alpha}(x) D_{l}^{\mu}\left(\kappa \mathbf{B}_{f}\right), \\
& <e_{f}^{\alpha}, e_{\beta}^{f}>=<e^{\alpha}, e_{\beta}>=\delta_{\beta}^{\alpha} .
\end{aligned}
$$

So, Minkowskian metric is written $\eta^{\alpha \beta}=\operatorname{diag}(1,-1,-1,-1)=<e_{f}^{\alpha}, e_{f}^{\beta}>=<e^{\alpha}, e^{\beta}>$.

\section{Reflection Matrix}

One interesting offshoot of general gauge principle is a formalism of reflection. A straightforward calculation for fields $s=0,1$ gives the explicit unitary reflection matrix

$$
R\left(x, x_{f}\right)=R_{f}\left(x_{f}\right) R_{g}(x)=\exp \left(-i \Theta_{f}\left(x_{f}\right)-\Theta_{g}(x)\right)
$$

provided

$$
\Theta_{f}\left(x_{f}\right)=g \int_{0}^{x_{f}} \mathbf{B}_{l}\left(x_{f}\right) d x_{f}^{l}, \quad \Theta_{g}(x)=\int_{0}^{x}\left[R_{f}^{+} \Gamma_{\mu} R_{f}+\psi^{-1} \partial_{\mu} \psi\right] d x^{\mu}, \quad \psi \equiv\left(\psi_{l}^{\mu}\right),
$$

where $\Theta_{g}=0$ for scalar field and $\Theta_{g}+\Theta_{g}^{+}=0$ for vector field, because of $\Gamma_{\mu}+\Gamma_{\mu}^{+}=0$ and $\partial_{\mu}\left(\psi^{-1} \psi\right)=0$. The function $S\left(B_{f}\right)$ has a form eq. $(2.11)$, since $S\left(B_{f}\right)=\frac{1}{4} R^{+}\left(\psi_{\mu}^{l} D_{l}^{\mu}\right) R=$ $\frac{1}{4} \psi_{\mu}^{l} D_{l}^{\mu}$. The infinitesimal gauge transformation $U_{f} \approx 1-i T^{a} \theta_{f}^{a}\left(x_{f}\right)$ yields

$$
U_{R}=\exp \left(i g C^{a b c} T^{a} \int_{0}^{x_{f}} \theta_{f}^{b} B_{l}^{c} d x_{f}^{l}+\Theta_{g}^{\prime}-\Theta_{g}\right)
$$

where the infinitesimal transformation ${B^{\prime}}_{l}^{a}=B_{l}^{a}+C^{a b c} \theta_{f}^{b} B_{l}^{c}-\frac{1}{g} \partial_{l}^{f} \theta_{f}^{a}$ was used. For example, a Lagrangian of isospinor-scalar shadow field $\varphi_{f}$ is in the form

$$
\begin{aligned}
& L_{f}\left(x_{f}\right)=J_{\psi} L(x)=J_{\psi}\left[\left(e^{\mu} \nabla_{\mu} \varphi\right)^{+}\left(e^{\mu} \nabla_{\mu} \varphi\right)-m^{2} \varphi^{+} \varphi\right]= \\
& =J_{\psi}\left[S\left(B_{f}\right)^{2}\left(D_{l} \varphi_{f}\right)^{+}\left(D_{l} \varphi_{f}\right)-m^{2} \varphi_{f}^{+} \varphi_{f}\right] .
\end{aligned}
$$


A Lagrangian of isospinor-vector Maxwell 's shadow field arises in a straightforward manner

$$
L_{f}\left(x_{f}\right)=J_{\psi} L(x)=-\frac{1}{4} J_{\psi} F_{\mu \nu}^{+} F^{\mu \nu}=-\frac{1}{4} J_{\psi} S\left(B_{f}\right)^{2}\left(F_{\mu \nu}^{(f)}\right)^{+} F_{(f)}^{\mu \nu},
$$

provided

$$
F_{\mu \nu}=\nabla_{\mu} A_{\nu}-\nabla_{\nu} A_{\mu}, \quad F_{\mu \nu}^{(f)}=\left(\psi_{\nu}^{k} D_{\mu}^{f}-\psi_{\mu}^{k} D_{\nu}^{f}\right) A_{k}^{f}, \quad D_{\mu}^{f}=D_{\mu}^{l} D_{l},
$$

with an additional gauge violating term

$$
\begin{aligned}
& L_{G}^{f}\left(x_{f}\right)=J_{\psi} L_{G}(x)=-\frac{1}{2} \zeta_{0}^{-1} J_{\psi}\left(\nabla_{\mu} A^{\mu}\right)^{+} \nabla_{\nu} A^{\nu}= \\
& =-\frac{1}{2} \zeta_{0}^{-1} J_{\psi} S\left(B_{f}\right)^{2}\left(\lambda_{m}^{l} D_{l} A_{f}^{m}\right)^{+} \lambda_{n}^{k} D_{k} A_{f}^{n}, \quad \lambda_{m}^{l}=\frac{1}{2}\left(\delta_{m}^{l}+\omega_{m}^{l}\right),
\end{aligned}
$$

where $\zeta_{0}^{-1}$ is gauge fixation parameter. Finally, a Lagrangian of isospinor-ghost fields is written

$$
L_{g h}^{f}\left(x_{f}\right)=J_{\psi} L_{g h}(x)=J_{\psi}<\left(e^{\mu} \partial_{\mu} C\right)^{+}, e^{\nu} \partial_{\nu} C>=J_{\psi} S\left(B_{f}\right)^{2}\left(D_{l} C_{f}\right)^{+} D_{l} C_{f} .
$$

Continuing along this line we come to a discussion of the reflection of spinor field $\Psi_{f}\left(x_{f}\right)(s=$ $\left.\frac{1}{2}\right)$ as a solution of eq. $(2.15)$

$$
\begin{aligned}
& \Psi(x)=R\left(\mathbf{B}_{f}\right) \Psi_{f}\left(x_{f}\right), \quad \bar{\Psi}(x)=\bar{\Psi}_{f}\left(x_{f}\right) \widetilde{R}^{+}\left(\mathbf{B}_{f}\right), \\
& g^{\mu}(x) \nabla_{\mu} \Psi(x)=S\left(B_{f}\right) R\left(\mathbf{B}_{f}\right) \gamma^{l} D_{l} \Psi_{f}\left(x_{f}\right), \\
& \left(\nabla_{\mu} \bar{\Psi}(x)\right) g^{\mu}(x)=S\left(B_{f}\right)\left(D_{l} \bar{\Psi}_{f}\left(x_{f}\right)\right) \gamma^{l} \widetilde{R}^{+}\left(\mathbf{B}_{f}\right),
\end{aligned}
$$

where $\widetilde{R}=\gamma^{0} R \gamma^{0}, \quad \Sigma^{\alpha \beta}=\frac{1}{4}\left[\gamma^{\alpha}, \gamma^{\beta}\right], \quad \Gamma_{\mu}(x)=\frac{1}{4} \Delta_{\mu, \alpha \beta}(x) \gamma^{\alpha} \gamma^{\beta}, \Delta_{\mu, \alpha \beta}(x)$ are Ricci rotation coefficients. The reflection matrix $R$ is in the form eq.(3.1), provided we make change

$$
\Theta_{g}(x)=\frac{1}{2} \int_{0}^{x} R_{f}^{+}\left\{g^{\mu} \Gamma_{\mu} R_{f}, g_{\nu} d x^{\nu}\right\}
$$

the $\{$,$\} is an anticommutator. A calculation now gives$

$$
S\left(B_{f}\right)=\frac{1}{8 K} \psi_{\mu}^{l}\left\{\widetilde{R}^{+} g^{\mu} R, \gamma_{l}\right\}=i n v
$$

where

$$
\begin{aligned}
& K=\widetilde{R}^{+} R=\widetilde{R}_{g}^{+} R_{g}= \\
& =\exp \left(-\frac{1}{2} \int_{0}^{x}\left(\left\{R_{f}^{+} \widetilde{\Gamma}_{\mu}^{+} g^{\mu}, g_{\nu} d x^{\nu}\right\} R_{f}+R_{f}^{+}\left\{g^{\mu} \Gamma_{\mu} R_{f}, g_{\nu} d x^{\nu}\right\}\right)\right), \quad \widetilde{\Gamma}_{\mu}^{+}=\gamma^{0} \Gamma_{\mu}^{+} \gamma^{0} .
\end{aligned}
$$

Taking into account that $\left[R_{f}, g_{\nu}\right]=0$ and substituting [13]

$$
\widetilde{\Gamma}_{\mu}^{+} g^{\nu}+g^{\nu} \Gamma_{\mu}=-\nabla_{\mu} g^{\nu}=0
$$

into eq.(3.12), we get

$$
K=1 \text {. }
$$


Since

$$
\widetilde{U}_{R}^{+} U_{R}=\widetilde{R} U_{f}^{+} \widetilde{R}^{\prime} R^{\prime} U_{f} R^{+}=\widetilde{R} U_{f}^{+} \widetilde{R}^{+} R U_{f} R^{+}
$$

where $\widetilde{R}^{\prime} R^{\prime}=\widetilde{R}^{+} R=1$, then

$$
\widetilde{U}_{R}^{+} U_{R}=\gamma^{0} U_{R}^{+} \gamma^{0} U_{R}=1
$$

A Lagrangian of isospinor-spinor shadow field may be written

$$
\begin{aligned}
& L_{f}\left(x_{f}\right)=J_{\psi} L(x)=J_{\psi}\left\{\frac{i}{2}\left[\bar{\Psi}(x) g^{\mu}(x) \nabla_{\mu} \Psi(x)-\left(\nabla_{\mu} \bar{\Psi}(x)\right) g^{\mu}(x) \Psi(x)\right]-m \bar{\Psi}(x) \Psi(x)\right\}= \\
& =J_{\psi}\left\{S\left(B_{f}\right) \frac{i}{2}\left[\bar{\Psi}_{f} \gamma^{l} D_{l} \Psi_{f}-\left(D_{l} \bar{\Psi}_{f}\right) \gamma^{l} \Psi_{f}\right]-m \bar{\Psi}_{f} \Psi_{f}\right\} .
\end{aligned}
$$

In special case, namely the curvature tensor $R_{\mu \nu \tau}^{\lambda}=0$, eq.(2.8) may be satisfied globally in $M^{4}$ by putting

$$
\psi_{l}^{\mu}=D_{l}^{\mu}=V_{l}^{\mu}=\frac{\partial x^{\mu}}{\partial \xi^{l}}, \quad\|D\| \neq 0, \quad \chi_{l}^{f}=0,
$$

where $\xi^{l}$ are inertial coordinates. So, $S=J_{\psi}=1$, which means that one simply has constructed local G-gauge theory in $M^{4}$ both in curvilinear as well as inertial coordinates.

\section{Action Principle}

Field equations may be inferred from an invariant action

$$
S=S_{B_{f}}+S_{\Phi}=\int L_{B_{f}}\left(x_{f}\right) d^{4} x_{f}+\int \sqrt{ } L_{\Phi}(x) d^{4} x .
$$

A Lagrangian $L_{B_{f}}\left(x_{f}\right)$ of gauge field $\mathbf{B}_{l}\left(x_{f}\right)$ defined on $M^{4}$ is invariant under Lorentz as well as $\mathrm{G}$-gauge groups. But a Lagrangian of the rest of fields $\Phi(x)$ defined on $R^{4}$ is invariant under the gauge group of gravitation $G_{R}$. Consequently, the whole action eq.(4.1) is $\mathrm{G}$-gauge invariant, since $G_{R}$ generated by $\mathrm{G}$. Field equations followed at once in terms of Euler-Lagrang variations respectively in $M^{4}$ and $R^{4}$

$$
\begin{aligned}
& \frac{\delta^{f} L_{B_{f}}}{\delta^{f} B_{l}^{a}}=J_{a}^{l}=-\frac{\delta^{f} L_{\Phi_{f}}}{\delta^{f} B_{l}^{a}}=-\frac{\partial g^{\mu \nu}}{\partial B_{l}^{a}} \frac{\delta\left(\sqrt{ } L_{\Phi}\right)}{\delta g^{\mu \nu}}= \\
& =-\frac{1}{2} \frac{\partial g^{\mu \nu}}{\partial B_{l}^{a}} \frac{\sqrt{ }}{\|D\|} \frac{D_{k \mu} \delta\left(\sqrt{ } L_{\Phi}\right)}{\delta D_{k}^{\nu}}=-\frac{\sqrt{ }}{2} \frac{\partial g^{\mu \nu}}{\partial B_{l}^{a}} T_{\mu \nu}, \\
& \frac{\delta L_{\Phi}}{\delta \Phi}=0, \quad \frac{\delta L_{\Phi}}{\delta \bar{\Phi}}=0,
\end{aligned}
$$

where $T_{\mu \nu}$ is the energy -momentum tensor of fields $\Phi(x)$. Making use of Lagrangian of corresponding shadow fields $\Phi_{f}\left(x_{f}\right)$, in generalized sense, one may readily define the energy -momentum conservation laws and also exploit whole advantages of field theory in terms of flat space in order to settle or mitigate the difficulties whenever they arise including the quantization of gravitation, which will not concern us here. Meanwhile, of course, one is free to carry out an inverse reflection to $R^{4}$ whenever it will be needed. To render our discussion here more transparent, below we clarify a relation between gravitational and coupling constants. So, we consider the theory at the limit of Newton 's 
non -relativistic law of gravitation in the case of static weak gravitational field given by Poisson equation [11]

$$
\nabla^{2} g_{00}=8 \pi G T_{00}
$$

In linear approximation

$$
g_{00}\left(\kappa \mathbf{B}_{f}\right) \approx 1+2 \kappa B_{k}^{a}\left(x_{f}\right) \theta_{a}^{k}, \quad \theta_{a}^{k}=\left(\frac{\partial D_{0}^{0}\left(\kappa \mathbf{B}_{f}\right)}{\partial \kappa B_{k}^{a}}\right)_{0}=\mathrm{const}
$$

then

$$
\theta_{a}^{k} \nabla^{2} \kappa B_{k}^{a}=4 \pi G T_{00}
$$

Since, the eq.(4.2) must match onto eq.(4.5) at considered limit, then the right -hand sides of both equations should be in the same form

$$
\kappa \theta_{a}^{k} \frac{\delta^{f} L_{B_{f}}}{\delta^{f} B_{a}^{k}}=\kappa \theta_{a}^{k} J_{k}^{a} \approx-\frac{\kappa^{2}}{2} \frac{\theta_{a}^{k} \partial g^{00}}{\partial\left(\kappa B_{a}^{k}\right)} T_{00}=\kappa^{2}\left(\theta_{a}^{k} \theta_{k}^{a}\right) T_{00} .
$$

With this final detail carried for one gets

$$
G=\frac{\kappa^{2}}{4 \pi \theta_{c}^{2}}, \quad \theta_{c}^{2} \equiv\left(\theta_{a}^{k} \theta_{k}^{a}\right)
$$

At this point we emphasize that Weinberg 's argumentation [14], namely, a prediction of attraction between particle and antiparticle, and repulsion between the same kind of particles, which is valid for pure vector theory, no longer holds in suggested theory of gravitation. Although the $\mathbf{B}_{l}\left(x_{f}\right)$ is vector gauge field, the eq.(4.7) just furnished the prove that only gravitational attraction is existed. One final observation is worth recording. A fascinating opportunity has turned out in the case if one utilizes reflected Lagrangian of Einstein 's gravitational field

$$
L_{B_{f}}\left(x_{f}\right)=J_{\psi} L_{E}(x)=\frac{J_{\psi} R}{16 \pi G},
$$

where $R$ is a scalar curvature. Hence, one readily gets the field equation

$$
\left(R_{\lambda}^{\mu}-\frac{1}{2} \delta_{\lambda}^{\mu} R\right) D_{\mu}^{l} \frac{\partial D_{l}^{\lambda}}{\partial B_{k}^{a}}=-8 \pi G U_{a}^{k}\left(\kappa \mathbf{B}_{f}\right)=-8 \pi G T_{\lambda \nu} D^{\nu l} \frac{\partial D_{l}^{\lambda}}{\partial B_{k}^{a}},
$$

which obviously leads to Einsten 's equation. Of course in these circumstances it is straightforward to choose $g^{\mu \nu}$ as the characteristic of gravitational field without referring to gauge field $B_{l}^{a}\left(x_{f}\right)$. However, in this case the energy -momentum tensor of gravitational field is well -defined. At this vital point suggested theory strongly differs from Einstein's classical theory. In line with this, one should use the real -curvilinear coordinates, in which the gravitational field was well -defined in the sense that it cannot be destroyed globally by coordinate transformations. While, taking into account general rules eq.(2.12), the energy -momentum tensor of gravitational field may be readily obtained by expressing the energy -momentum tensor of vector gauge field $B_{l}^{a}\left(x_{f}\right)$ in terms of metric tensor and its derivatives

$$
\begin{aligned}
& T_{\mu \nu}=g_{\mu \lambda} T_{\nu}^{\lambda}=g_{\mu \lambda} \psi_{k}^{\lambda} \psi_{\nu}^{i} T_{(f) i}^{k}= \\
& =g_{\mu \lambda} \psi_{k}^{\lambda} \psi_{\nu}^{i}\left(\frac{\partial B_{l}^{a}}{\partial g^{\mu^{\prime} \nu^{\prime}}} \psi_{i}^{\sigma} \partial_{\sigma} g^{\mu^{\prime} \nu^{\prime}} \frac{\partial\left(\partial_{\tau} g^{\lambda^{\prime} \tau^{\prime}}\right)}{\partial\left(\partial_{k}^{f} B_{l}^{a}\right)} \frac{\partial\left(J_{\psi} L_{E}\right)}{\partial\left(\partial_{\tau} g^{\lambda^{\prime} \tau^{\prime}}\right)}-\delta_{i}^{k} J_{\psi} L_{E}\right) .
\end{aligned}
$$


At last, we should note that eq.(4.8) is not the simplest one among gauge invariant Lagrangians. Moreover, it must be the same in all cases including eq.(3.18) too. But in last case it contravenes the standard gauge theory. There is no need to contemplate such a drastic revision of physics. So, for our part we prefer $\mathrm{G}$-gauge invariant Lagrangian in terms of $M^{4}$

$$
L_{B_{f}}\left(x_{f}\right)=-\frac{1}{4}<\mathbf{F}_{l k}^{f}\left(x_{f}\right), \mathbf{F}_{f}^{l k}\left(x_{f}\right)>_{K},
$$

where $\mathbf{F}_{l k}^{f}\left(x_{f}\right)$ is in the form eq. $(2.7),<,>_{K}$ is the Killing undegenerate form on the Lie algebra of group $\mathrm{G}$ for adjoint representation. Certainly, an explicit form of functions $D_{l}^{\mu}\left(\kappa \mathbf{a}_{f}\right)$ should be defined.

\section{$5 \quad$ Gravitation at $G=U^{l o c}(1)$}

The gravitational interaction with hidden Abelian local group $G=U^{l o c}(1)=S O^{l o c}(2)$ and one-dimensional trivial algebra $\hat{g}=R^{1}$ was considered in [1-5], wherein the explicit form of transformation function $D_{l}^{\mu}$ is defined by making use of principle bundle $p: E \rightarrow G(2.3)$. It is worthwhile to consider the major points of it anew in concise form and make it complete by calculations, which will be adjusted to fit the outlined here theory. We start with a very brief recapitulation of structure of flat manifold $G(2.3)={ }^{*} R^{2} \otimes R^{3}=R_{+}^{3} \oplus R_{-}^{3}$ provided with the basis vectors $e_{(\lambda \alpha)}^{0}=O_{\lambda} \otimes \sigma_{\alpha}$, where $<O_{\lambda}, O_{\tau}>={ }^{*} \delta_{\lambda \tau}=1-\delta_{\lambda \tau}$, $<\sigma_{\alpha}, \sigma_{\beta}>=\delta_{\alpha \beta}(\lambda, \tau= \pm: \alpha, \beta=1,2,3), \delta$ is Kronecker symbol. A bilinear form on vector fields of sections $\tau$ of tangent bundle of $G(2.3): \hat{g}^{0}: \tau \otimes \tau \rightarrow C^{\infty}(G(2.3))$, namely, the metric $\left(g_{(\lambda \alpha)(\tau \beta)}^{0}\right)$ is a section of conjugate vector bundle with components $\hat{g}^{0}\left(e_{(\lambda \alpha)}^{0}, e_{(\tau \beta)}^{0}\right)$ in basis $\left(e_{(\lambda \alpha)}^{0}\right)$. The $G(2.3)$ decomposes into three-dimensional ordinary $\left(R_{f}^{3}\right)$ and time $\left(T_{f}^{3}\right)$ flat spaces $G(2.3)=R_{f}^{3} \oplus T_{f}^{3}$ with signatures $\operatorname{sgn}\left(R_{f}^{3}\right)=(+++)$ and $\operatorname{sgn}\left(T_{f}^{3}\right)=(---)$. Since all directions in $T_{f}^{3}$ are equivalent, then by notion time one implies the projection of time-coordinate on fixed arbitrary universal direction in $T_{f}^{3}$. By this reduction $T_{f}^{3} \rightarrow T_{f}^{1}$ the transition $G(2.3) \rightarrow M^{4}=R_{f}^{3} \oplus T_{f}^{1}$ may be performed whenever it will be needed. Under massless gauge field $a_{(\lambda \alpha)}\left(\eta_{f}\right)$ associating with $U^{l o c}(1)$ the basis $e_{(\lambda \alpha)}^{0}$ transformed at a point $\eta_{f} \in G(2.3)$ according to eq.(2.3)

$$
e_{(\lambda \alpha)}=D_{(\lambda \alpha)}^{(\tau \beta)} e_{(\tau \beta)}^{0}
$$

While, the matrix $D$ is in the form $D=C \otimes R$, where the distortion transformations $O_{(\lambda \alpha)}=C_{(\lambda \alpha)}^{\tau} O_{\tau}$ and $\sigma_{(\lambda \alpha)}=R_{(\lambda \alpha)}^{\beta} O_{\beta}$ are defined. Thereby $C_{(\lambda \alpha)}^{\tau}=\delta_{\lambda}^{\tau}+\kappa a_{(\lambda \alpha)}{ }^{*} \delta_{\lambda}^{\tau}$, but $R$ is a matrix of the group $S O(3)$ of all ordinary rotations of the planes, each of which involves two arbitrary basis vectors of $R_{\lambda}^{3}$, around the orthogonal axes. The angles of permissible rotations will be determined throughwith a special constraint imposed upon distortion transformations, namely, a sum of distortions of corresponding basis vectors $O_{\lambda}$ and $\sigma_{\beta}$ has to be zero at given $\lambda$ :

$$
<O_{(\lambda \alpha)}, O_{\tau}>_{\tau \neq \lambda}+\frac{1}{2} \varepsilon_{\alpha \beta \gamma} \frac{<\sigma_{(\lambda \beta)}, \sigma_{\gamma}>}{<\sigma_{(\lambda \beta)}, \sigma_{\beta}>}=0
$$

where $\varepsilon_{\alpha \beta \gamma}$ is an antisymmetric unit tensor. There up on $\tan \theta_{(\lambda \alpha)}=-\kappa a_{(\lambda \alpha)}$, where $\theta_{(\lambda \alpha)}$ is the particular rotation around the axis $\sigma_{\alpha}$ of $R_{\lambda}^{3}$. Inasmuch as the $R$ should be independent of sequence of rotation axes, then it implies the mean value $R=\frac{1}{6} \sum_{i \neq j \neq k} R^{(i j k)}$, 
where $R^{(i j k)}$ the matrix of rotations carried out in sequence $(i j k)(i, j, k=1,2,3)$. As it was seen at the outset the field $a_{(\lambda \alpha)}$ was generated by the distortion of basis pseudo-vector $O_{\lambda}$, when the distortion of $\sigma_{\alpha}$ has followed from eq(5.2).

Certainly, the whole theory outlined in sections (1-4) will then hold provided we simply replace each single index $\mu$ of variables by the pair $(\lambda \alpha)$ and so on. Following to standard rules, next we construct the diffeomorphism $\eta^{(\lambda \alpha)}\left(\eta_{f}^{(\tau \beta)}\right): G(2.3) \rightarrow G(23)$ and introduce the action eq.(4.1) for the fields. In the sequel, a transition from six-dimensional curved manifold $G(23)$ to four-dimensional Riemannian geometry $R^{4}$ is straightforward by making use of reduction of three time-components $e_{0 \alpha}=\frac{1}{\sqrt{2}}\left(e_{(+\alpha)}+e_{(-\alpha)}\right)$ of basis six-vectors $e_{(\lambda \alpha)}$ to single $e_{0}$ in fixed universal direction. Actually, since Lagrangian of fields on $R^{4}$ is a function of scalars, namely, $A_{(\lambda \alpha)} B^{(\lambda \alpha)}=A_{0 \alpha} B^{0 \alpha}+A_{\alpha} B^{\alpha}$, so taking into account that $A_{0 \alpha} B^{0 \alpha}=A_{0 \alpha}<e^{0 \alpha}, e^{0 \beta}>B_{0 \beta}=A_{0}<e^{0}, e^{0}>B_{0}=A_{0} B^{0}$, one readily may perform a required transition. The gravitation field equation is written

$$
\partial_{f}^{(\tau \beta)} \partial_{(\tau \beta)}^{f} a^{(\lambda \alpha)}-\left(1-\zeta_{0}^{-1}\right) \partial_{f}^{(\lambda \alpha)} \partial_{(\tau \beta)}^{f} a^{(\tau \beta)}=-\frac{1}{2} \sqrt{-g} \frac{\partial g^{(\tau \beta)(\mu \gamma)}}{\partial a_{(\lambda \alpha)}} T_{(\tau \beta)(\mu \gamma)}
$$

To render our discussion more transparent, below we consider in detail a solution of spherical-symmetric static gravitational field $a_{(+\alpha)}=a_{(-\alpha)}=\frac{1}{\sqrt{2}} a_{0 \alpha}\left(r_{f}\right)$. So, $\theta_{(+\alpha)}=$ $\theta_{(-\alpha)}=-\arctan \left(\frac{\kappa}{\sqrt{2}} a_{0 \alpha}\right)$ and $\sigma_{(+\alpha)}=\sigma_{(-\alpha)}$. It is convenient to make use of spherical coordinates $\sigma_{(+1)}=\sigma_{r}, \sigma_{(+2)}=\sigma_{\theta}, \sigma_{(+3)}=\sigma_{\varphi}$. The transition $G(2.3) \rightarrow M^{4}$ is performed by choosing in $T_{f}^{3}$ the universal direction along radius-vector: $x_{f}^{0 r}=t_{f}, x_{f}^{0 \theta}=x_{f}^{0 \varphi}=0$. Then, from eq.(5.1) one gets

$$
e_{0}=D_{0}^{0} e_{0}^{0}, \quad e_{r}=D_{r}^{r} e_{r}^{0}, \quad e_{\theta}=e_{\theta}^{0}, \quad e_{\varphi}=e_{\varphi}^{0}
$$

provided $D=C \otimes I$ with components $D_{0}^{0}=1+\frac{\kappa}{\sqrt{2}} a_{0}, D_{r}^{r}=1-\frac{\kappa}{\sqrt{2}} a_{0}, a_{0} \equiv a_{0 r}$, where $e_{0}^{0}=\xi_{0} \otimes \sigma_{r}, \quad e_{r}^{0}=\xi \otimes \sigma_{r}, \quad e_{\theta}^{0}=\xi \otimes \sigma_{\theta}, \quad e_{\varphi}^{0}=\xi \otimes \sigma_{\varphi}, \xi_{0}=\frac{1}{\sqrt{2}}\left(O_{+}+O_{-}\right)$and $\xi=\frac{1}{\sqrt{2}}\left(O_{+}-O_{-}\right)$. The coordinates $x^{\mu}(t, r, \theta, \varphi)$ implying $x^{\mu}\left(x_{f}^{l}\right): M^{4} \rightarrow R^{4}$ exist in the whole region $p^{-1}(U) \in R^{4}$

$$
\frac{\partial x^{\mu}}{\partial x_{f}^{l}}=\psi_{l}^{\mu}=\frac{1}{2}\left(D_{l}^{\mu}+\omega_{l}^{m} D_{m}^{\mu}\right)
$$

where according to eq.(5.4) one has $x^{0 r}=t, x^{0 \theta}=x^{0 \varphi}=0$. A straightforward calculation gives non-vanishing components

$$
\begin{aligned}
& \psi_{0}^{0}=\frac{1}{2} D_{0}^{0}, \quad \psi_{1}^{0}=\frac{1}{2} t_{f} \partial_{r}^{f} D_{0}^{0}, \quad \psi_{1}^{1}=D_{r}^{r}, \quad \psi_{2}^{2}=\psi_{3}^{3}=0, \\
& \omega_{1}^{1}=\omega_{2}^{2}=\omega_{3}^{3}=1, \quad \omega_{1}^{0}=t_{f} \partial_{r}^{f} D_{0}^{0} .
\end{aligned}
$$

Although $\partial_{r} \psi_{0}^{0}=\Gamma_{01}^{0} \psi_{0}^{0}$ and $\partial_{r} \psi_{1}^{1}=\Gamma_{11}^{1} \psi_{1}^{1}$, but

$$
\partial_{t} \psi_{1}^{0}=\psi_{0}^{0} \partial_{r}^{f} D_{0}^{0} \neq \Gamma_{10}^{0} \psi_{0}^{0}=\psi_{0}^{0} \partial_{r} \ln D_{0}^{0},
$$


where according to eq.(5.4), the non-vanishing components of Christoffel symbol are written $\Gamma_{01}^{0}=\frac{1}{2} g^{00} \partial_{r} g_{00}, \Gamma_{00}^{1}=-\frac{1}{2} g^{11} \partial_{r} g_{00}, \Gamma_{11}^{1}=\frac{1}{2} g^{11} \partial_{r} g_{11}$. So, the condition eq. (2.13) holds, namely, the curvature of $R^{4}$ is not zero. The curved space $R^{4}$ has the group of motions $S O(3)$ with two- dimensional space-like orbits $S^{2}$ where the standard coordinates are $\theta$ and $\varphi$. The stationary subgroup of $S O(3)$ acts isotropically upon the tangent space at the point of sphere $S^{2}$ of radius $r$. So, the bundle $p: R^{4} \rightarrow R^{2}$ has the fiber $S^{2}=p^{-1}(x)$, $x \in R^{4}$ with a trivial connection on it, where $R^{2}$ is the factor-space $R^{4} / S O(3)$. In outside of the distribution of matter with the total mass $M$, the eq.(5.3) in Feynman gauge reduced to $\nabla_{f}^{2} a_{0}=0$, which has the solution $\frac{\kappa}{\sqrt{2}} a_{0}=-\frac{G M}{r_{f}}=-\frac{r_{g}}{2 r_{f}}$ (see eq.(4.7)). So, the line element is written

$$
d s^{2}=\left(1-\frac{r_{g}}{2 r_{f}}\right)^{2} d t^{2}-\left(1+\frac{r_{g}}{2 r_{f}}\right)^{2} d r^{2}-r^{2}\left(\sin ^{2} \theta d \varphi^{2}+d \theta^{2}\right)
$$

provided by eq.(5.5) and eq.(5.6).Finally, for example, the explicit form of unitary matrix $U_{R}$ of gravitation group $G_{R}$ in the case of scalar field reads

$$
U_{R}=R^{\prime} U_{f} R^{+}=e^{-i\left[t_{f} \partial_{r}^{f} \theta_{f}\left(r_{f}\right)+\theta_{f}\left(r_{f}\right)\right]}
$$

where

$$
R=R_{f}=e^{-\frac{i g r_{g}}{2 r_{f}} t_{f}}, \quad g\left(a_{0}^{\prime}-a_{0}\right)=\partial_{r}^{f} \theta_{f}\left(r_{f}\right) .
$$

A Lagrangian of charged scalar shadow field eq.(3.4) is in the form

$$
L_{f}\left(x_{f}\right)=\frac{1}{2}\left[\left(\frac{7}{8}\right)^{2}\left(D_{l} \varphi_{f}\right)^{*} D_{l} \varphi_{f}-m^{2} \varphi_{f}^{*} \varphi_{f}\right]
$$

where, according to eq.(2.11) and eq.(5.6), one has $J_{\Psi}=\frac{1}{2}\left(1+\left\|\omega_{l}^{m}\right\|\right)=\frac{1}{2}, S=\frac{7}{8}$.

\section{Distortion of Flat Manifold $G(2.2 .3)$ at $G=U^{l o c}(1)$}

Following to [1-5], the foregoing theory can be readily generalized for the distortion of 12dimensional flat manifold $G(2.2 .3)={ }^{*} R^{2} \otimes{ }^{*} R^{2} \otimes R^{3}=\underset{\eta}{G}(2.3) \oplus \underset{u}{G}(2.3)=\sum_{\lambda, \mu=1}^{2} \oplus R_{\lambda \mu}^{3}=$ ${ }_{x}^{R^{3}} \oplus \underset{x}{T^{3}} \oplus \underset{u}{R^{3}} \oplus \underset{u}{T^{3}}$ with corresponding basis vectors $e_{(\lambda, \mu, \alpha)}=O_{\lambda, \mu} \otimes \sigma_{\alpha} \subset G(2.2 .3)$, $\stackrel{i}{i(\lambda \alpha)}^{0}=O_{i_{\lambda}} \otimes \sigma_{\alpha} \subset \underset{i}{G}(2.3)$, where $O_{i+}=\frac{1}{\sqrt{2}}\left(O_{1,1}+\varepsilon_{i} O_{2,1}\right),{\underset{i}{-}}_{-}=\frac{1}{\sqrt{2}}\left(O_{1,2}+\varepsilon_{i} O_{2,2}\right), \varepsilon_{\eta}=1$, $\varepsilon_{u}=-1$ and $<O_{\lambda, \mu}, O_{\tau, \nu}>={ }^{*} \delta_{\lambda \tau}{ }^{*} \delta_{\mu \nu}(\lambda, \mu, \tau, \nu=1,2),<\sigma_{\alpha}, \sigma_{\beta}>=\delta_{\alpha \beta}$. There up on $<\underset{i_{\lambda}}{O}, O_{i_{\tau}}>=\varepsilon_{i} \delta_{i j}{ }^{*} \delta_{\lambda \tau}$. The massless gauge field of distortion $a_{(\lambda, \mu, \alpha)}\left(\zeta_{f}\right)$ with the values in Lie algebra of $U^{l o c}(1)$ is a local form of expression of connection in principle bundle $p: E \rightarrow G(2.2 .3)$ with a structure group $U^{l o c}(1)$. Collection of matter fields $\Phi_{f}\left(\zeta_{f}\right)$ are the sections of vector bundles associated with $U^{l o c}(1)$ by reflection $\Phi_{f}: G(2.2 .3) \rightarrow E$ that $p \Phi_{f}\left(\zeta_{f}\right)=\zeta_{f}$, where the coordinates $\zeta^{(\lambda, \mu, \alpha)}$ exist in the whole region $p^{-1}\left(U^{(f)}\right) \in G(2.2 .3)$. 
Outlined theory will then hold, while each pair of indices $(\lambda \alpha)$ will be replaced by the $(\lambda, \mu, \alpha)$. So the basis $e_{(\tau, \nu, \beta)}$ transformed

$$
e_{(\lambda \mu \alpha)}=D_{(\lambda \mu \alpha)}^{(\tau, \nu, \beta)} e_{(\tau, \nu, \beta)},
$$

provided $D=C \otimes R$, where $O_{(\lambda \mu \alpha)}=C_{(\lambda \mu \alpha)}^{\tau, \nu} O_{\tau, \nu}, \sigma_{(\lambda \mu \alpha)}=R_{(\lambda \mu \alpha)}^{\beta} \sigma_{\beta}$. The matrices $C$ generate the group of distortion transformations of bi-pseudo-vectors $O_{\tau, \nu}: C_{(\lambda \mu \alpha)}^{\tau, \nu}=$ $\delta_{\lambda}^{\tau} \delta_{\mu}^{\nu}+\kappa a_{(\lambda, \mu, \alpha)} * \delta_{\lambda}^{\tau *} \delta_{\mu}^{\nu}$, but the matrices $R$ are the elements of the group $S O(3)_{\lambda \mu}$ of ordinary rotations of the planes of corresponding basis vectors of $R_{\lambda \mu}^{3}$. A special constraint eq.(5.2) holds for basis vectors of each $R_{\lambda \mu}^{3}$. Thus, the gauge field $a_{(\lambda, \mu, \alpha)}$ was generated by the distortion of bi-pseudo-vectors $O_{\tau, \nu}$. While the rotation transformations $R$ follow due to eq.(5.2) and $R$ implies, as usual, the mean value with respect to sequence of rotation axes. The angles of permissible rotations are $\tan \theta_{(\lambda, \mu, \alpha)}=-\kappa a_{(\lambda, \mu, \alpha)}\left(\zeta_{f}\right)$. The action eq.(4.1) now reads

$$
S=S_{a_{f}}+S_{\Phi}=\int L_{a_{f}}\left(\zeta_{f}\right) d \zeta^{(1,1,1)} \wedge \cdots \wedge d \zeta^{(2,2,3)}+\int \sqrt{g} L_{\Phi}(\zeta) d \zeta^{(111)} \wedge \cdots \wedge d \zeta^{(223)},
$$

where $g$ is the determinant of metric tensor on $G(223)$, while the $\zeta^{(\lambda \mu \alpha)}\left(\zeta^{(\tau, \nu, \beta)}\right): G(2.2 .3) \rightarrow$ $G(223)$ was constructed according to eq.(2.8)-eq.(2.10), provided

$$
\frac{\partial \zeta^{(\lambda \mu \alpha)}}{\partial \zeta^{(\tau, \nu, \beta)}}=\psi_{(\tau, \nu, \beta)}^{(\lambda \mu \alpha)}=\frac{1}{2}\left(D_{(\tau, \nu, \beta)}^{(\lambda \mu \alpha)}+\omega_{(\tau, \nu, \beta)}^{(\rho, \omega, \gamma)} D_{(\rho, \omega, \gamma)}^{(\lambda \mu \alpha)}\right)
$$

Right through the variational calculations one infers the field equations eq.(4.2), while

$$
\begin{aligned}
& \partial^{(\tau, \nu, \beta)} \partial_{(\tau, \nu, \beta)} a^{(\lambda, \mu, \alpha)}-\left(1-\zeta_{0}^{-1}\right) \partial^{(\lambda, \mu, \alpha)} \partial_{(\tau, \nu, \beta)} a^{(\tau, \nu, \beta)}=J^{(\lambda, \mu, \alpha)}= \\
& =-\frac{1}{2} \sqrt{g} \frac{g^{(\tau \nu \beta)(\rho \omega \gamma)}}{\partial a_{(\lambda, \mu, \alpha)}} T_{(\tau \nu \beta)(\rho \omega \gamma)},
\end{aligned}
$$

provided

$$
T_{(\tau \nu \beta)(\rho \omega \gamma)}=\frac{2 \delta\left(\sqrt{g} L_{\Phi}\right)}{\sqrt{g} \delta g^{(\tau \nu \beta)(\rho \omega \gamma)}} .
$$

The curvature of manifold $\underset{i}{G}(2.3) \rightarrow \underset{i}{G}(23)$ (sec. 5), which leads to four-dimensional Riemannian geometry $R^{4}$, is a familiar distortion

$$
a_{(1,1, \alpha)}=a_{(2,1, \alpha)} \equiv \frac{1}{\sqrt{2}{ }^{\eta}(+\alpha)}, \quad a_{(1,2, \alpha)}=a_{(2,2, \alpha)} \equiv \frac{1}{\sqrt{2}} a_{(-\alpha)}^{a},
$$

when $\sigma_{(11 \alpha)}=\sigma_{(21 \alpha)} \equiv \sigma_{(+\alpha)}, \sigma_{(12 \alpha)}=\sigma_{(22 \alpha)} \equiv \sigma_{(-\alpha)}$. Hence $\underset{i_{(\lambda \alpha)}}{e}=O_{i(\lambda \alpha)} \otimes \sigma_{(\lambda \alpha)}$, where $\underset{i(\lambda \alpha)}{O_{i}}=O_{i}+\frac{\kappa}{\sqrt{2}} \varepsilon_{i} a_{\eta_{(\lambda \alpha)}} * \delta_{\lambda}^{\tau} O_{i_{\tau}}$. In the aftermath $G(223)=\underset{\eta}{G}(23) \oplus \underset{u}{G}(23)$. The other important case of inner-distortion

$$
a_{(1,1, \alpha)}=-a_{(2,1, \alpha)} \equiv \frac{1}{\sqrt{2}} a_{(+\alpha)}^{a}, \quad a_{(1,2, \alpha)}=-a_{(2,2, \alpha)} \equiv \frac{1}{\sqrt{2}^{u}} a_{(-\alpha)}
$$

leads to $\sigma_{(11 \alpha)}=-\sigma_{(21 \alpha)} \equiv \sigma_{(+\alpha)}, \sigma_{(12 \alpha)}=-\sigma_{(22 \alpha)} \equiv \sigma_{(-\alpha)}$. Hence, ${\underset{i}{(\lambda \alpha)}}_{i \lambda}=O_{i}+$ $\frac{\kappa}{\sqrt{2}} \varepsilon_{i} a_{u_{(\lambda \alpha)}}{ }^{\tau} \delta_{\lambda}^{\tau} O_{i \tau}$, where the $e_{i(\lambda \alpha)}=O_{i(\lambda \alpha)} \otimes \sigma_{(\lambda \alpha)}$ is the basis in inner-distorted manifold $\underset{i}{G}(23)$.

\section{Part II. Regular Goyaks}




\section{The Goyaks and Link-Establishing Processes}

Next we develop the foundations for our viewpoint and proceed to general definitions and conjectures of the theory of goyaks directly. Henceforth with in last two parts it is convenient to describe the theory in terms of manifold $G(2.2 .3)$. But, surely, one may readily perform a transition to $M^{4}$ (sec.5,6) whenever it will be needed. We choose a simple setting and consider new formations designed to endow certain physical properties and satisfying the general rules stated below. At this, we may consider it briefly hoping to mitigate a shortage of insufficient rigorous treatment by the further exposition of the theory and make them complete and discussed in broad sense in due course.

Conjecture 1 The 6-dimensional basis vectors $\underset{i_{(\lambda \alpha)}}{e_{(\lambda \alpha}}$ (or co-vectors $\underset{i_{0}}{e^{(\lambda \alpha)}}$ ) we explore from a specific novel point of view, as being the main characteristics of the real existing structures called "goyaks". Below we distinguish two type of goyaks: $\eta$-type $(i=\eta)$ and u-type $(i=u)$, respectively.

Conjecture 2 The goyaks establish reciprocal "linkage" between themselves. The links

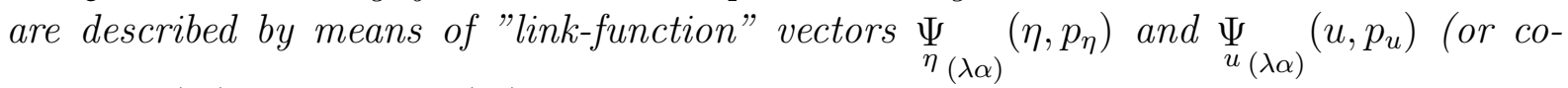
vectors $\underset{\eta}{\Psi}(\lambda \alpha)\left(\eta, p_{\eta}\right)$ and $\left.\underset{u}{\Psi}(\lambda \alpha)\left(u, p_{u}\right)\right)$ :

$$
\underset{\eta_{( \pm \alpha)}}{\Psi}\left(\eta, p_{\eta}\right)=\eta_{( \pm \alpha)} \underset{\eta_{ \pm}}{\Psi}\left(\eta, p_{\eta}\right), \quad \underset{u( \pm \alpha)}{\Psi}\left(u, p_{u}\right)=u_{( \pm \alpha)} \underset{u_{ \pm}}{\Psi}\left(u, p_{u}\right)
$$

where the 6-vectors of 'link-coordinates" $-\eta, u$, and "link-momenta" - $p_{\eta}, p_{u}$ respectively are $\eta=\eta_{\eta_{0}}^{(\lambda \alpha)} \eta_{(\lambda \alpha)}, \quad p_{\eta}={\underset{\eta}{0}}_{\eta_{(\lambda \alpha)}^{(\lambda \alpha)}} \eta_{(\lambda \alpha}, \quad u=e_{u_{0}}^{(\lambda \alpha)} u_{(\lambda \alpha)}, \quad p_{u}=\underset{u_{0}}{e_{u(\lambda \alpha)}^{(\lambda \alpha)} p}$. In words, the probability of finding the goyak in the state with fixed link-coordinate and momentum is determined by the square of its state wave function $\underset{\eta_{ \pm}}{\Psi}\left(\eta, p_{\eta}\right)$, or $\underset{u_{ \pm}}{\Psi}\left(u, p_{u}\right)$. This provides a simple intuitive meaning of state link-functions.

Conjecture 3 Characterizing by the basis vectors $\underset{i(\lambda \alpha)}{{\underset{i}{(\lambda)}}^{0}}$ and link-functions eq.(7.1), the goyaks are called "regular" ones. The "distorted" goyaks are described in terms of distorted basis vectors $e_{i(\lambda \alpha)}^{e}(\theta)=O_{i(\lambda \alpha)}(\theta) \otimes \sigma_{(\lambda \alpha)}(\theta)$ (see Part III) and distorted link-functions $\Psi_{i(\lambda \alpha)}(\theta)$, where $\theta(\eta, u)=\left\{\underset{i_{(\lambda \alpha)}}{\left\{\theta^{\prime}\right.}\right\}$ are the angles of distortion transformations.

Conjecture 4 The $\eta$-type goyak may accept the linkage only from u-type goyak, which is described by the link-function $\Psi_{(\lambda \alpha)}\left(\eta, p_{\eta}\right)$ ( $\eta$-channel). The u-type goyak, in its turn, may accept the linkage only from $\eta$-type goyak, to which the link-function $\underset{u_{(\lambda \alpha)}}{\Psi}\left(u, p_{u}\right)$ (u-channel) is corresponded. It is assumed that the link-establishing processes between the goyaks of the same type are absent. 
Conjecture 5 The simplest system made of two goyaks of different types becomes stable only due to the reciprocal link-establishing processes in the case of stable linkage. The latter is valid if only the subsidiary condition of link-stability holds

$$
\left|p_{\eta}\right|=\left(p_{\eta}^{(\lambda \alpha)}, p_{\eta(\lambda \alpha)}\right)^{1 / 2}=\left|p_{u}\right|=\left(p_{u}^{(\lambda \alpha)}, p_{u(\lambda \alpha)}\right)^{1 / 2} .
$$

Otherwise they are unstable.

Conjecture 6 There is not any restriction on the number of goyaks of both types getting into the link-establishing processes simultaneously. In order to be a stable system the link-stability condition must be held for each linkage separately.

Conjecture 7 The persistent processes of creation and annihilation of goyaks occur in the different states $s, s^{\prime}, s^{\prime \prime}, \ldots$ It is assumed that the "creation" of the goyak in given state $(s)$ is stimulated by its transition to this state from the other states $\left(s^{\prime}, s^{\prime \prime}, \ldots\right)$, while the "annihilation" means a vice versa. Along many states $\left(s, s^{\prime}, s^{\prime \prime}, \ldots\right)$ there is a lowest one $\left(s_{0}\right)$, in which all goyaks are regular. In other higher states the goyaks are distorted. Thus, meanwhile the transition from the state $\left(s_{0}\right)$ to any other state $(s)$ and vice versa the goyak undergoes to distortion transformation.

Conjecture 8 Hereinafter we will be interested only by a special stable system of regular goyaks, which is formed in lowest state $\left(s_{0}\right)$ and made of only one goyak of $\eta$-type and infinite number of u-type goyaks. They are characterized by the identical basis structure vectors. The $\eta$-type goyak is called "fundamental", but the u-type goyaks - "ordinary" ones.

Conjecture 9 The dimension of the basis elements may be directly regarded as the dimension of associated goyak. In general, it can be an arbitrary number. But in outlined theory a reduction of the goyak's dimension just to six $(\lambda= \pm ; \quad \alpha=1,2,3)$ is backed up by the arguments are briefly stated as follows: It is taken for granted that the infinite sequence of random transitions along many configurations of goyaks $(\lambda=1, \ldots ; \quad \alpha=1, \ldots)$ in an arbitrary given state are the random vector variables with the differential distribution function $\varphi(\nu)=\nu^{n} e^{-\pi \nu^{2}}$, provided by the frequency of the transitions $\nu$. At $n \gg 1$ expected mean frequency of transition from the state $n$ is given by

$$
m(n)=\frac{\int_{0}^{\infty} \nu^{n} \exp ^{\left(-\pi \nu^{2}\right)} d \nu}{\int_{0}^{\infty} \exp ^{\left(-\pi \nu^{2)}\right.} d \nu}=\frac{\Gamma\left(\frac{n+1}{2}\right)}{2 \pi^{(n+1) / 2}} .
$$

The most probable number of dimension of goyak is achieved at the minimum of the function $m(n)$. The inverse value of $m(n)$ is isomorphic to the value function of $(n+1)$ dimensional hypersphere of unit radius. This function is unimodal for the positive values of $(n+1)$ and it is of indefinite sign for negative values of $(n+1)$. The maximum extension volume of the formation is achieved at $n= \pm 6$ [15]. Therefore the maximum probability corresponds to six-dimensional goyaks. That is why only six-dimensional goyaks $(\lambda=$ $\pm ; \quad \alpha=1,2,3)$ would be considered below. 


\section{Operator Manifold $\hat{G}(2.2 .3)$}

The processes of creation and annihilation of regular goyaks in the lowest state $\left(s_{0}\right)$ may be described by the formalism similar to secondary quantization, but in the same time it will be the appropriate expansion over the geometric structures such as goyaks. We first deal with a substitution of the basis elements of goyak's structure by the corresponding operators of creation and annihilation of the regular goyaks acting in the configuration space of occupation numbers. Instead of pseudo-vectors we introduce the following operators supplied by additional index $(r)$ referring to the quantum numbers of corresponding state

$$
\begin{aligned}
& \hat{O}_{1}^{r}=O_{1}^{r} \alpha_{1}, \quad \hat{O}_{2}^{r}=O_{2}^{r} \alpha_{2}, \quad \hat{O}_{r}^{\lambda}={ }^{*} \delta^{\lambda \mu} \hat{O}_{\mu}^{r}=\left(\hat{O}_{\lambda}^{r}\right)^{+}, \\
& \left\{\hat{O}_{\lambda}^{r}, \hat{O}_{\tau}^{r^{\prime}}\right\}=\delta_{r r^{\prime}}{ }^{*} \delta_{\lambda \tau} I_{2}, \quad<O_{\lambda}^{r}, O_{\tau}^{r^{\prime}}>=\delta_{r r^{\prime}}{ }^{*} \delta_{\lambda \tau}, \quad I_{2}=\left(\begin{array}{cc}
1 & 0 \\
0 & 1
\end{array}\right) .
\end{aligned}
$$

The matrices $\alpha_{\lambda}$ satisfy the conditions

$$
\alpha^{\lambda}={ }^{*} \delta^{\lambda \mu} \alpha_{\mu}=\left(\alpha_{\lambda}\right)^{+}, \quad\left\{\alpha_{\lambda}, \alpha_{\tau}\right\}={ }^{*} \delta_{\lambda \tau} I_{2}
$$

For example, they can be in the form $\alpha_{1}=\left(\begin{array}{ll}0 & 1 \\ 0 & 0\end{array}\right), \quad \alpha_{2}=\left(\begin{array}{ll}0 & 0 \\ 1 & 0\end{array}\right)$. This forms the starting point for quantization. Creation operator $\hat{O}_{1}^{r}$ generates one-occupied state $\left|1>_{(0)} \equiv\right| 0, \ldots, 1, \ldots>_{(0)}$ and the basis vector $O_{1}^{r}$ with the quantum number $r$, right through acting on non-occupied vacuum state $\left|0>_{(0)} \equiv\right| 0,0, \ldots>_{(0)}$ :

$$
\hat{O}_{1}^{r}\left|0>_{(0)}=O_{1}^{r}\right| 1>_{(0)} .
$$

Accordingly, the action of annihilation operator $\hat{O}_{2}^{r}$ on one-occupied state yields the vacuum state and the basis vector $O_{2}^{r}$

$$
\hat{O}_{2}^{r}\left|1>_{(0)}=O_{2}^{r}\right| 0>_{(0)} .
$$

So define $\hat{O}_{1}^{r}\left|1>_{(0)}=0, \quad \hat{O}_{2}^{r}\right| 0>_{(0)}=0$. The matrix realization of the states $\mid 0>_{0}$ and $\mid 1>_{0}$, for instance, may be as follows: $\left|0>_{0} \equiv \chi_{1}=\left(\begin{array}{l}0 \\ 1\end{array}\right), \quad\right| 1>_{0} \equiv \chi_{2}=\left(\begin{array}{l}1 \\ 0\end{array}\right)$. The operator of occupation number is $\hat{N}_{r}^{(0)}=\hat{O}_{1}^{r} \hat{O}_{2}^{r}$, with the expectation values implying Pauli's exclusion principle $_{(0)}<0\left|\hat{N}_{r}^{(0)}\right| 0>_{(0)}=0, \quad{ }_{(0)}<1\left|\hat{N}_{r}^{(0)}\right| 1>_{(0)}=1$. The vacuum state reads $\chi_{0} \equiv \mid 0>_{(0)}=\prod_{r=1}^{N}\left(\chi_{1}\right)_{r}$. With this final detail cared for one-occupied state takes the form $\chi_{r^{\prime}} \equiv \mid 1>_{(0)}=\left(\chi_{2}\right)_{r^{\prime}} \prod_{r \neq r^{\prime}}\left(\chi_{1}\right)_{r}$. Continuing along this line, instead of ordinary vectors we introduce the operators $\hat{\sigma}_{\alpha}^{r} \equiv \delta_{\alpha \beta \gamma} \sigma_{\beta}^{r} \widetilde{\sigma}_{\gamma}$, where $\widetilde{\sigma}_{\gamma}$ are Pauli's matrices, and

$$
<\sigma_{\alpha}^{r}, \sigma_{\beta}^{r^{\prime}}>=\delta_{r r^{\prime}} \delta_{\alpha \beta}, \quad \hat{\sigma}_{r}^{\alpha}=\delta^{\alpha \beta} \hat{\sigma}_{\beta}^{r}=\left(\hat{\sigma}_{\alpha}^{r}\right)^{+}=\hat{\sigma}_{\alpha}^{r}, \quad\left\{\hat{\sigma}_{\alpha}^{r}, \hat{\sigma}_{\beta}^{r^{\prime}}\right\}=2 \delta_{r r^{\prime}} \delta_{\alpha \beta} I_{2} .
$$

For the vacuum state $\mid 0>_{(\sigma)} \equiv \varphi_{1(\alpha)}$ and one-occupied state $\mid 1_{(\alpha)}>_{(\sigma)} \equiv \varphi_{2(\alpha)}$ we make use of matrix realization $\varphi_{1(\alpha)} \equiv \chi_{1}, \quad \varphi_{2(1)}=\left(\begin{array}{l}1 \\ 0\end{array}\right), \quad \varphi_{2(2)}=\left(\begin{array}{r}-i \\ 0\end{array}\right), \quad \varphi_{2(3)}=\left(\begin{array}{r}0 \\ -1\end{array}\right)$. Then

$$
\hat{\sigma}_{\alpha}^{r} \varphi_{1(\alpha)}=\sigma_{\alpha}^{r} \varphi_{2(\alpha)}=\left(\sigma_{\alpha}^{r} \widetilde{\sigma}_{\alpha}\right) \varphi_{1(\alpha)}, \quad \hat{\sigma}_{\alpha}^{r} \varphi_{2(\alpha)}=\sigma_{\alpha}^{r} \varphi_{1(\alpha)}=\left(\sigma_{\alpha}^{r} \widetilde{\sigma}_{\alpha}\right) \varphi_{2(\alpha)} .
$$


Hence, the single eigen-value $\left(\sigma_{\alpha}^{r} \widetilde{\sigma}_{\alpha}\right)$ has associated with it quite different $\varphi_{\lambda(\alpha)}$. The eigen-value is degenerated with degeneracy degree equal 2. Due to it, along many quantum numbers $r$ there is also the quantum number of the spin $\vec{\sigma}$ with the values $\sigma_{3}=\frac{1}{2} s \quad(s=$ $\pm 1)$. This rule for spin quantum number is not without an important reason. The argument for this conclusion is compulsory suggested by the properties of operators $\hat{\sigma}_{\alpha}^{r}$. As we will see later on, this consequently gives rise to spin of particle.

One-occupied state reads $\varphi_{r^{\prime}(\alpha)}=\left(\varphi_{2(\alpha)}\right)_{r^{\prime}} \prod_{r \neq r^{\prime}}\left(\chi_{1}\right)_{r}$. Next we introduce the operators

$$
\hat{\gamma}_{(\lambda, \mu, \alpha)}^{r} \equiv \hat{O}_{\lambda}^{r_{1}} \otimes \hat{O}_{\mu}^{r_{2}} \otimes \hat{\sigma}_{\alpha}^{r_{3}}, \quad \hat{\gamma}_{r}^{(\lambda, \mu, \alpha)} \equiv \hat{O}_{r_{1}}^{\lambda} \otimes \hat{O}_{r_{2}}^{\mu} \otimes \hat{\sigma}_{r_{3}}^{\alpha}={ }^{*} \delta^{\lambda \tau *} \delta^{\mu \nu} \delta^{\alpha \beta} \hat{\gamma}_{(\tau, \nu, \beta)}^{r},
$$

and also the state vector

$$
\chi_{\lambda, \mu, \tau(\alpha)} \equiv \mid \lambda, \mu, \tau(\alpha)>=\chi_{\lambda} \otimes \chi_{\mu} \otimes \varphi_{\tau(\alpha)},
$$

where $\lambda, \mu, \tau, \nu=1,2 ; \quad \alpha, \beta=1,2,3$ and $r \equiv\left(r_{1}, r_{2}, r_{3}\right)$. Hence $\hat{\gamma}_{(\lambda, \mu, \alpha)}^{r} \chi_{\tau, \nu, \delta(\beta)}=$ $\left(\hat{O}_{\lambda}^{r_{1}} \chi_{\tau}\right) \otimes\left(\hat{O}_{\mu}^{r_{2}} \chi_{\nu}\right) \otimes\left(\hat{\sigma}_{\alpha}^{r_{3}} \varphi_{\delta(\beta)}\right)$. Omitting the two-valuedness of state vector we apply $|\lambda, \tau, \delta(\beta)>\equiv| \lambda, \tau>$, and the same time remember that always the summation must be extended over the double degeneracy of the spin states $(s= \pm 1)$.

With this final detail cared for one infers the explicit forms of corresponding matrix elements:

$$
\begin{aligned}
& <2,2\left|\hat{\gamma}_{(1,1, \alpha)}^{r}\right| 1,1>=e_{(1,1, \alpha)}^{r}, \quad<1,1\left|\hat{\gamma}_{r}^{(1,1, \alpha)}\right| 2,2>=e_{r}^{(1,1, \alpha)}, \\
& <2,1\left|\hat{\gamma}_{(1,2, \alpha)}^{r}\right| 1,2>=e_{(1,2, \alpha)}^{r}, \quad<1,2\left|\hat{\gamma}_{r}^{(1,2, \alpha)}\right| 2,1>=e_{r}^{(1,2, \alpha)}, \\
& <1,2\left|\hat{\gamma}_{(2,1, \alpha)}^{r}\right| 2,1>=e_{(2,1, \alpha)}^{r}, \quad<2,1\left|\hat{\gamma}_{r}^{(2,1, \alpha)}\right| 1,2>=e_{r}^{(2,1, \alpha)}, \\
& <1,1\left|\hat{\gamma}_{(2,2, \alpha)}^{r}\right| 2,2>=e_{(2,2, \alpha)}^{r}, \quad<2,2\left|\hat{\gamma}_{r}^{(2,2, \alpha)}\right| 1,1>=e_{r}^{(2,2, \alpha)}
\end{aligned}
$$

The operators of occupation numbers are

$$
\begin{aligned}
& \hat{N}_{1 \alpha \beta}^{r r^{\prime}}=\hat{\gamma}_{(1,1, \alpha)}^{r} \hat{\gamma}_{(2,2, \beta)}^{r^{\prime}}=\hat{N}_{r r^{\prime}}^{\alpha \beta}=\hat{\gamma}_{r}^{(2,2, \alpha)} \hat{\gamma}_{r^{\prime}}^{(1,1, \beta)}, \\
& \hat{N}_{2 \alpha \beta}^{r r^{\prime}}=\hat{\gamma}_{(2,1, \alpha)}^{r} \hat{\gamma}_{(1,2, \beta)}^{r^{\prime}}={\underset{N}{2 r r^{\prime}}}_{\hat{N}^{\alpha \beta}}=\hat{\gamma}_{r}^{(1,2, \alpha)} \hat{\gamma}_{r^{\prime}}^{(2,1, \beta)},
\end{aligned}
$$

with the expectation values implying Pauli's exclusion principle

$$
\begin{aligned}
& <2,2\left|\underset{1_{r r^{\prime}}}{\hat{N}^{\alpha \beta}}\right| 2,2>=\delta_{r r^{\prime}} \delta_{\alpha \beta}, \quad<1,2\left|\underset{2}{\hat{N}_{r r^{\prime}}}\right| 1,2>=\delta_{r r^{\prime}} \delta_{\alpha \beta}, \\
& <1,1\left|\underset{1_{r r^{\prime}}}{\hat{N}_{\alpha \beta}}\right| 1,1>=0, \quad<2,1\left|\underset{2}{\hat{N}_{r r^{\prime}}^{\alpha \beta}}\right| 2,1>=0 .
\end{aligned}
$$

The set of operators $\left\{\hat{\gamma}_{(\lambda, \mu, \alpha)}^{r}\right\}$ eq. (8.7) is the basis in operator manifold $\hat{G}(2.2 .3)={ }^{*} \hat{R}^{22} \otimes$ $\hat{R}^{3}$. Here ${ }^{*} \hat{R}^{22}$ is the $2 \times 2$-dimensional linear bi-pseudo operator-space, with the set of the linear unit operator bi-pseudo vectors $\left\{\hat{O}_{\lambda, \mu}^{r_{1} r_{2}} \equiv \hat{O}_{\lambda}^{r_{1}} \otimes \hat{O}_{\mu}^{r_{2}}\right\}$, and $\hat{R}^{3}$ is the three-dimensional real linear operator-space with the basis consisted of the ordinary unit operator-vectors $\left\{\hat{\sigma}_{\alpha}^{r}\right\}$. By means of mathematical apparatus, which is in close analogy to differential geometry [9], a general description of operator manifold $\hat{G}(2.2 .3)$ may be presented. Bilinear form on operator-vectors $\hat{\Phi}(\zeta)=\hat{\gamma}_{r}^{(\lambda, \mu, \alpha)} \Phi_{(\lambda, \mu, \alpha)}^{r}(\zeta) \in \hat{G}(2.2 .3)$ reads in component form $\hat{g}=\hat{g}_{r r^{\prime}}^{(\lambda, \mu, \alpha)(\tau, \nu, \beta)} d \Phi_{(\lambda, \mu, \alpha)}^{r}(\zeta) \otimes d \Phi_{(\tau, \nu, \beta)}^{r^{\prime}}(\zeta)$. Analogical form on operator-co-vectors 
$\overline{\hat{\Phi}}(\zeta)=\hat{\gamma}_{(\lambda, \mu, \alpha)}^{r} \Phi_{r}^{(\lambda, \mu, \alpha)}(\zeta) \in \hat{G}(2.2 .3)$, can be written, where $\Phi_{r}^{(\lambda, \mu, \alpha)}(\zeta)=\bar{\Phi}_{(\lambda, \mu, \alpha)}^{r}(\zeta)$. One easily gets

$$
\begin{aligned}
& <1,1|\hat{\Phi}(\zeta) \overline{\hat{\Phi}}(\zeta)| 1,1>=\Phi_{(1,1, \alpha)}^{r}(\zeta) \Phi_{r}^{(1,1, \alpha)}(\zeta) \\
& <1,2|\hat{\Phi}(\zeta) \hat{\hat{\Phi}}(\zeta)| 1,2>=\Phi_{(1,2, \alpha)}^{r}(\zeta) \Phi_{r}^{(1,2, \alpha)}(\zeta) \\
& <2,1|\hat{\Phi}(\zeta) \overline{\hat{\Phi}}(\zeta)| 2,1>=\Phi_{(2,1, \alpha)}^{r}(\zeta) \Phi_{r}^{(2,1, \alpha)}(\zeta) \\
& <2,2|\hat{\Phi}(\zeta) \overline{\hat{\Phi}}(\zeta)| 2,2>=\Phi_{(2,2, \alpha)}^{r}(\zeta) \Phi_{r}^{(2,2, \alpha)}(\zeta)
\end{aligned}
$$

Introducing the new state vectors

$$
\begin{aligned}
& \chi^{0}\left(\nu_{1}, \nu_{2}, \nu_{3}, \nu_{4}\right)=\left|1,1>^{\nu_{1}} \cdot\right| 1,2>^{\nu_{2}} \cdot\left|2,1>^{\nu_{3}} \cdot\right| 2,2>^{\nu_{4}}, \\
& \nu_{i}= \begin{cases}1 & \text { if } \nu=\nu_{i} \\
0 & \text { otherwise, for some } i,\end{cases} \\
& \mid \chi_{-}(\lambda)>=\left\{\begin{array}{ll}
\chi^{0}(1,0,0,0) & \lambda=1, \\
\chi^{0}(0,0,1,0) & \lambda=2,
\end{array} \quad \mid \chi_{+}(\lambda)>= \begin{cases}\chi^{0}(0,0,0,1) & \lambda=1, \\
\chi^{0}(0,1,0,0) & \lambda=2,\end{cases} \right.
\end{aligned}
$$

provided

$$
\begin{aligned}
& <\lambda, \mu,\left|\tau, \nu>=\delta_{\lambda \tau} \delta_{\mu \nu}, \quad<\chi_{ \pm}\right| A\left|\chi_{\mp}>=\sum_{\lambda}<\chi_{ \pm}(\lambda)\right| A \mid \chi_{\mp}(\lambda)> \\
& <\chi_{ \pm}(\lambda)\left|\chi_{ \pm}(\mu)>=\delta_{\lambda \mu}, \quad<\chi_{ \pm}\right| A\left|\chi_{ \pm}>=\sum_{\lambda}<\chi_{ \pm}(\lambda)\right| A \mid \chi_{ \pm}(\lambda)> \\
& <\chi_{ \pm}(\lambda) \mid \chi_{\mp}(\mu)>=0
\end{aligned}
$$

we get the matrix elements as follows:

$$
\begin{aligned}
& <\chi_{-}|\hat{\Phi}(\zeta) \overline{\hat{\Phi}}(\zeta)| \chi_{-}>\equiv \Phi_{-}^{2}(\zeta)=\Phi_{(1,1, \alpha)}^{r}(\zeta) \Phi_{r}^{(1,1, \alpha)}(\zeta)+\Phi_{(2,1, \alpha)}^{r}(\zeta) \Phi_{r}^{(2,1, \alpha)}(\zeta) \\
& <\chi_{+}|\hat{\Phi}(\zeta) \Phi(\zeta)| \chi_{+}>\equiv \Phi_{+}^{2}(\zeta)=\Phi_{(2,2, \alpha)}^{r}(\zeta) \Phi_{r}^{(2,2, \alpha)}(\zeta)+\Phi_{(1,2, \alpha)}^{r}(\zeta) \Phi_{r}^{(1,2, \alpha)}(\zeta) .
\end{aligned}
$$

The operator manifold decomposes $\hat{G}(2.2 .3)=\hat{G}(2.3) \oplus \underset{u}{\hat{G}}(2.3)$, where $\underset{i}{\hat{G}}(2.3) \quad(i=\eta, u)$ is the six-dimensional operator manifold with the basis $\left\{\underset{i(\lambda \alpha)}{\hat{\gamma}^{r}}\right\} \quad(\lambda= \pm ; \quad \alpha=1,2,3 ; \quad i=$

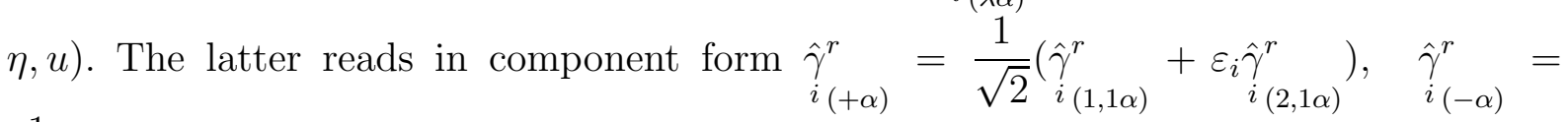
$\frac{1}{\sqrt{2}}\left(\hat{\gamma}_{i(1,2 \alpha)}^{r}+\varepsilon_{i} \hat{\gamma}_{i(2,2 \alpha)}^{r}\right)$. The expansions of operator-vectors $\underset{i}{\hat{\Psi}} \in \underset{i}{\hat{G}}(2.3)$ and operator-

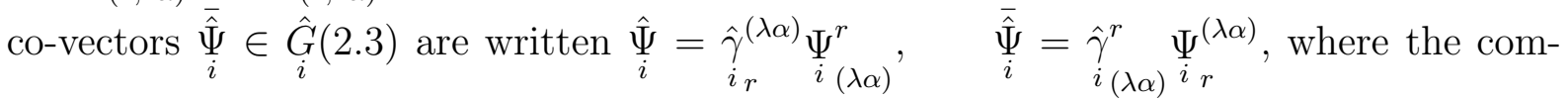

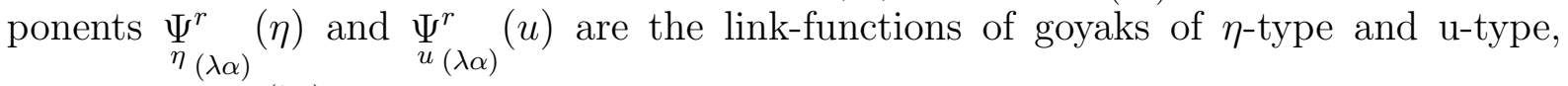
respectively: ${\underset{i}{r}}_{r}^{(\lambda \alpha)}=\bar{\Psi}_{i(\lambda \alpha)}^{r}$. The operator of occupation number of $i$-type goyak takes the form $\hat{N}_{r r^{\prime}}^{\alpha \beta}=\varepsilon_{i} \hat{\gamma}_{i_{r}}^{(-\alpha)} \hat{\gamma}_{i_{r^{\prime}}}^{(+\beta)}$, with corresponding expectation values

$$
<\chi_{-}\left|\underset{i_{r r^{\prime}}}{\hat{N}^{\alpha \beta}}\right| \chi_{-}>=0, \quad<\chi_{+}\left|\underset{i_{r r^{\prime}}}{\hat{N}^{\alpha \beta}}\right| \chi_{+}>=\varepsilon_{i}<\underset{i_{r}}{e^{(-\alpha)}} \underset{i_{r^{\prime}}}{e^{(+\beta)}}>=\delta_{r r^{\prime}} \delta_{\alpha \beta} .
$$

Taking into account two-valuedness of degenerate spin states it follows that the goyaks are the fermions with the half-integral spins. That is, the link-functions $\underset{\eta_{\lambda}}{\Psi}(\eta)$ and $\underset{u_{\lambda}}{\Psi}(u)$ can 
be regarded as the Fermi fields of $\eta$ - and $u$-type goyaks. Explicitly the matrix elements read

$$
\begin{aligned}
& \Phi_{-}^{2}(\zeta)=<\chi_{-}|\underset{\eta}{\hat{\Psi}}(\eta) \underset{\eta}{\underset{\hat{\Psi}}{\bar{N}}}(\eta)+\underset{u}{\hat{\Psi}}(u) \underset{u}{\overline{\hat{\Psi}}}(u)| \chi_{-}>=\varepsilon_{i} \underset{i}{\hat{\Psi}} \underset{(+\alpha)}{\hat{\Psi}_{i}^{(+\alpha)}}= \\
& =\hat{\Psi}_{(+\alpha)}(\eta){\underset{\eta}{(+\alpha)}}_{\overline{\hat{\Psi}}}(\eta)-\hat{\Psi}_{u(+\alpha)}(u){\underset{u}{(+\alpha)}}_{\overline{\hat{\Psi}}}(u), \\
& \Phi_{+}^{2}(\zeta)=<\chi_{+}|\underset{\eta}{\hat{\Psi}}(\eta) \underset{\eta}{\stackrel{\bar{\Psi}}{\bar{T}}}(\eta)+\underset{u}{\hat{\Psi}}(u) \underset{u}{\overline{\hat{\Psi}}}(u)| \chi_{+}>=\varepsilon_{i} \underset{i}{\hat{\Psi}} \underset{(-\alpha)}{\hat{\Psi}_{i}^{(-\alpha)}}= \\
& =\hat{\Psi}_{(-\alpha)}(\eta){\underset{\eta}{(-\alpha)}}_{\overline{\hat{\Psi}}}^{\chi_{(-\alpha)}}(\eta) \hat{\Psi}_{u(-\alpha)}(u) .
\end{aligned}
$$

\section{Quantum Field Aspect of Operator Manifold $\hat{G}(2.2 .3)$}

The nature of operator manifold $\hat{G}(2.2 .3)$ provides its elements with both field and geometric aspects. The field aspect will be the subject for discussion in this section. Our notation will be that of the textbook by [16]. As far as goyak field may be regarded as a fermion field, its description either regular or distorted was provided by the theory, which is in close analogy to Dirac's conventional wave-mechanical theory of fermions with spin $\frac{\overrightarrow{1}}{2}$ treated in terms of manifold $G(2.2 .3)$ [1-5]. The final formulation of quantum theory is equivalent to configuration space wave mechanics with antisymmetric state functions.

We remind that it is considered only the special system of regular goyaks, which is made of fundamental goyak of $\eta$-type and infinite number of $u$-type ordinary goyaks. They are characterized by the identical structure vectors. To become stable the goyaks in this system have established the stable linkage. The link-stability requirement holds for each ordinary goyak

$$
p^{2}=p_{\eta}^{2}-p_{u}^{2}=p_{\eta}^{(\lambda \alpha)} p_{(\lambda \alpha)}-{\underset{u}{(\lambda \alpha)}{ }_{u(\lambda \alpha)}}^{(\lambda \alpha}
$$

The regular goyak field may be considered as bi-spinor field $\Psi(\zeta)$ defined on manifold $G(2.2 .3)=\underset{\eta}{G}(2.3) \oplus \underset{u}{G}(2.3): \Psi(\zeta)=\underset{\eta}{\Psi}(\eta) \underset{u}{\Psi}(u)[1-5]$, where the $\underset{i}{\Psi}$ is a bi-spinor associated with the manifold $\underset{i}{G}(2.3)$. Lagrangian of free field is written down

$$
L=\frac{1}{2}\left\{\widetilde{\widetilde{\Psi}}(\zeta) \gamma^{(\lambda, \mu, \alpha)} \partial_{(\lambda, \mu, \alpha)} \widetilde{\Psi}(\zeta)-\partial_{(\lambda, \mu, \alpha)} \widetilde{\widetilde{\Psi}}(\zeta) \gamma^{(\lambda, \mu, \alpha)} \widetilde{\Psi}(\zeta)\right\}
$$

provided

$$
\begin{aligned}
& \widetilde{\Psi}(\zeta)=e \otimes \Psi(\zeta)=\left(\begin{array}{ll}
1 & 1 \\
1 & 1
\end{array}\right) \otimes \Psi(\zeta), \quad \widetilde{\bar{\Psi}}(\zeta)=\bar{\Psi}(\zeta) \otimes e, \quad \bar{\Psi}(\zeta)=\Psi^{+}(\zeta) \gamma^{0}, \\
& \gamma^{(\lambda, \mu, \alpha)}=\widetilde{O}^{\lambda} \otimes \widetilde{O}^{\mu} \otimes \widetilde{\sigma}^{\alpha}, \quad \partial_{(\lambda, \mu, \alpha)}=\partial / \partial \zeta^{(\lambda, \mu, \alpha)}, \quad \widetilde{O}^{\lambda}={ }^{*} \delta^{\lambda \mu} \widetilde{O}_{\mu}=\left(\widetilde{O}_{\lambda}\right)^{+}, \\
& \widetilde{O}_{1}=\frac{1}{\sqrt{2}}\left(\begin{array}{cr}
1 & 1 \\
-1 & -1
\end{array}\right), \quad \widetilde{O}_{2}=\frac{1}{\sqrt{2}}\left(\begin{array}{rr}
1 & -1 \\
1 & -1
\end{array}\right),
\end{aligned}
$$

and $\gamma^{0}, \quad \gamma^{\alpha}$ are Dirac's matrices in standard representation. Field equations follow at once right through the variational principle of least action

$$
\begin{aligned}
& \hat{p} \widetilde{\Psi}(\zeta)=i(\widetilde{\gamma} \partial) \widetilde{\Psi}(\zeta)=i \gamma^{(\lambda, \mu, \alpha)} \partial_{(\lambda, \mu, \alpha)} \widetilde{\Psi}(\zeta)=0, \\
& \widetilde{\bar{\Psi}}(\zeta) \hat{p}^{+}=-i \tilde{\bar{\Psi}}(\zeta)(\widetilde{\gamma} \partial)^{+}=-i \partial_{(\lambda, \mu, \alpha)}(\zeta) \gamma_{(\lambda, \mu, \alpha)}=0
\end{aligned}
$$


where $\left(\gamma^{(\lambda, \mu, \alpha)}\right)^{+}={ }^{*} \delta^{\lambda \tau *} \delta^{\mu \nu} \delta^{\alpha \beta} \gamma^{(\tau, \nu, \beta)}=\gamma_{(\lambda, \mu, \alpha)}$. Explicitly the eq.(9.4) reads

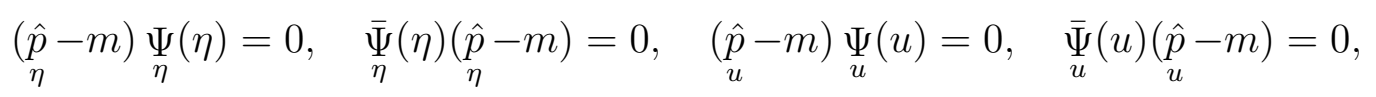

where the function of mass at rest of bi-spinor fields $\underset{\eta}{\Psi}$ and $\underset{u}{\Psi}$ is introduced $m \Psi(\zeta) \equiv \underset{u}{\hat{p}} \Psi(\zeta)=\underset{\eta}{\hat{p}} \Psi(\zeta)$ and

$$
\begin{aligned}
& \gamma_{\eta}^{(\lambda \alpha)}=\widetilde{O}_{\eta}^{\lambda} \otimes \widetilde{\sigma}^{\alpha}=\xi_{0} \otimes \gamma^{(\lambda \alpha)}=\xi_{0} \otimes \widetilde{O}^{\lambda} \otimes \widetilde{\sigma}^{\alpha}, \\
& \gamma_{u}^{\eta(\lambda \alpha)}=\widetilde{O}_{u}^{\lambda} \otimes \widetilde{\sigma}^{\alpha}=\xi \otimes \gamma^{(\lambda \alpha)}=\xi \otimes \widetilde{O}^{\lambda} \otimes \widetilde{\sigma}^{\alpha} \\
& \xi_{0}=\left(\begin{array}{rr}
1 & 0 \\
0 & -1
\end{array}\right) \quad \xi=\left(\begin{array}{cc}
0 & 1 \\
-1 & 0
\end{array}\right), \quad \xi_{0}^{2}=-\xi^{2}=1, \quad\left\{\xi_{0}, \xi\right\}=0 \\
& (\underset{i}{\gamma(\lambda \alpha)})^{+}=\varepsilon_{i}^{*} \delta^{\lambda \tau} \delta_{i}^{\alpha \beta} \underset{i}{\gamma(\tau \beta)}=\varepsilon_{i} \underset{i(\lambda \alpha)}{\gamma}, \quad \underset{\eta}{\hat{\partial}}=\gamma^{(\lambda \alpha)}{ }_{\eta}{ }_{(\lambda \alpha)}, \\
& \left(\gamma^{(\lambda \alpha)}\right)^{+}={ }^{*} \delta^{\lambda \tau} \delta^{\alpha \beta} \gamma^{(\tau \beta)}, \quad \underset{u}{\hat{\partial}}=\gamma^{(\lambda \alpha)} \underset{u(\lambda \alpha)}{\partial}, \quad \underset{\eta}{\hat{p}}=i \underset{\eta}{\partial}, \\
& \hat{p}=i \hat{\partial}_{u}, \quad{\underset{\eta}{(\lambda \alpha)}}_{\left(\lambda / \partial \eta^{(\lambda \alpha)},\right.}={ }_{u(\lambda \alpha)}=\partial / \partial u^{(\lambda \alpha)} .
\end{aligned}
$$

The state of free goyak of $i$-type with definite values of link-momentum $p_{i}$ and spin projection $s$ is described by means of plane waves, respectively (in units $\hbar=1, \quad c=1$ ):

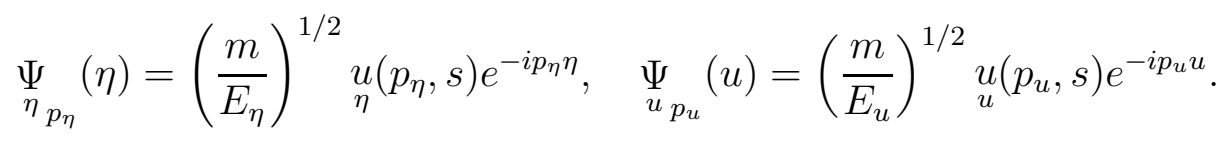

It is denoted $E_{i} \equiv p_{i_{0}}=\left(p_{i_{0 \alpha}}^{p}, p_{i_{0 \alpha}}^{p}\right)^{1 / 2}, \quad p_{i_{0 \alpha}}^{p}=\frac{1}{\sqrt{2}}\left(p_{i(+\alpha)}+p_{i_{(-\alpha)}}\right)$, and

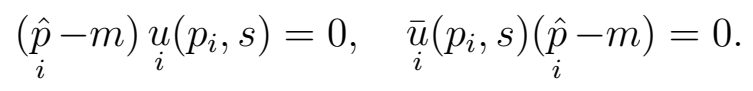

The amplitudes of waves $\underset{i}{u}\left(p_{i}, s\right)$ are normalized bi-spinors. It is necessary to consider also the solutions of negative frequencies

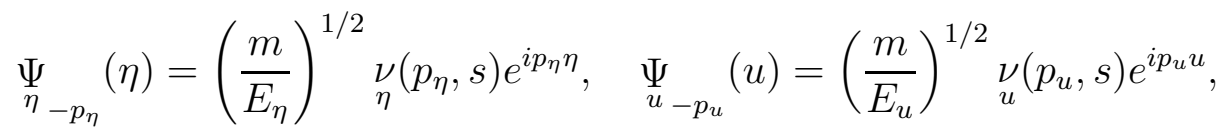

and

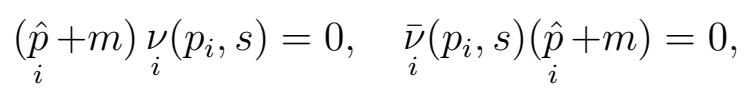

where $p_{i_{\alpha}}=\frac{1}{\sqrt{2}}\left(p_{i(+\alpha)}-p_{i(-\alpha)}\right), \quad p_{\eta}^{2}=E_{\eta}^{2}-\vec{p}_{\eta}^{2}=p_{u}^{2}=E_{u}^{2}-\vec{p}_{u}^{2}=m^{2}$. For the spinors the useful relations of orthogonality and completeness hold. Turning to secondary quantization of goyak's field we make use of localized wave packets constructed by means of superposition of plane wave solutions furnished by creation and annihilation operators in agreement with Pauli's principle

$$
\hat{\Psi}(\eta)=\sum_{ \pm s} \int \frac{d^{3} p_{\eta}}{(2 \pi)^{3 / 2}} \underset{\eta}{\hat{\Psi}}\left(p_{\eta}, s, \eta\right), \quad \underset{u}{\hat{\Psi}}(u)=\sum_{ \pm s} \int \frac{d^{3} p_{u}}{(2 \pi)^{3 / 2}} \underset{u}{\Psi}\left(p_{u}, s, u\right)
$$


where, as usual, it is denoted

$$
\begin{aligned}
& \hat{\Psi}\left(p_{\eta}, s, \eta\right)={\underset{\eta}{\hat{\gamma}}}^{(\lambda \alpha)}\left(p_{\eta}, s\right) \underset{\eta}{\Psi} \underset{(\lambda \alpha)}{\Psi}\left(p_{\eta}, s, \eta\right), \quad \underset{\eta}{\hat{\Psi}}\left(p_{\eta}, s, \eta\right)={\underset{\eta}{(\lambda \alpha)}}_{\hat{\gamma}}\left(p_{\eta}, s\right) \underset{\eta}{\Psi}(\lambda \alpha)\left(p_{\eta}, s, \eta\right),
\end{aligned}
$$

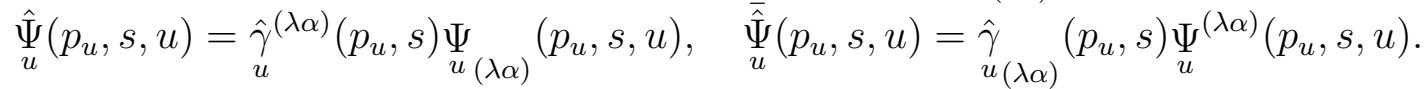

One has $\underset{\eta_{( \pm \alpha)}}{\Psi}=\eta_{( \pm \alpha)} \underset{\eta_{ \pm}}{\Psi}, \quad \underset{u}{\Psi} \underset{( \pm \alpha)}{\Psi}=u_{( \pm \alpha)} \underset{u \pm}{\Psi}, \quad \underset{\eta}{\Psi} \Psi^{( \pm \alpha)}=\eta^{( \pm \alpha)} \underset{\eta}{\Psi^{ \pm}}, \quad \underset{u}{\Psi}{ }^{( \pm \alpha)}=u^{( \pm \alpha)} \underset{u}{\Psi^{ \pm}}$, provided $\underset{i_{+}}{\Psi} \equiv{\underset{i}{p_{i}}}_{\Psi_{-}}, \quad \underset{i_{-}}{\Psi} \equiv \Psi_{-p_{i}}^{\Psi}, \quad \Psi_{i}^{\Psi^{\lambda}}=\bar{\Psi}_{i_{\lambda}}, \quad{\underset{i}{(}}^{(\lambda \alpha)}=\bar{\Psi}_{i_{(\lambda \alpha)}}$. A closer examination of the properties of the matrix elements of the anticommutators of expansion coefficients shows that

$$
\begin{aligned}
& \left.<\chi_{-} \mid \underset{i}{\left\{\hat{\gamma}^{(+\alpha)}\right.}\left(p_{i}, s\right),{\underset{j}{\gamma}(+\beta)}_{j}\left(p_{j}^{\prime}, s^{\prime}\right)\right\} \mid \chi_{-}>= \\
& =<\hat{e}_{i}^{(+\alpha)}\left(p_{i}, s\right), \hat{j}_{(+\beta)}\left(p_{j}^{\prime}, s^{\prime}\right)>=\varepsilon_{i} \delta_{i j} \delta_{s s^{\prime}} \delta_{\alpha \beta} \delta^{(3)}\left(\vec{p}_{i}-{\overrightarrow{p^{\prime}}}_{i}\right) \text {, } \\
& <\chi_{+}\left|\left\{\hat{\gamma}_{j}^{(-\beta)}\left(p_{j}^{\prime}, s^{\prime}\right), \hat{\gamma}_{i(-\alpha)}\left(p_{i}, s\right)\right\}\right| \chi_{+}>= \\
& =<\underset{j}{\hat{e}_{j}^{(-\beta)}}\left(p_{j}^{\prime}, s^{\prime}\right), \hat{e}_{i(-\alpha)}^{\hat{e}}\left(p_{i}, s\right)>=\varepsilon_{i} \delta_{i j} \delta_{s s^{\prime}} \delta_{\alpha \beta} \delta^{(3)}\left(\vec{p}_{i}-{\overrightarrow{p^{\prime}}}_{i}\right) .
\end{aligned}
$$

We can also consider the analogical wave packets of operator-vector fields of operator manifold $\hat{G}(2.2 .3)$. Explicitly the matrix element of anticommutator reads

$$
\begin{aligned}
& <\chi_{ \pm}\left|\left\{\hat{\gamma}^{(\lambda, \mu, \alpha)}(p, s), \hat{\gamma}_{(\tau, \nu, \beta)}\left(p^{\prime}, s^{\prime}\right)\right\}\right| \chi_{ \pm}>= \\
& =<e^{(\lambda, \mu, \alpha)}(p, s), e_{(\tau, \nu, \beta)}\left(p^{\prime}, s^{\prime}\right)>=\delta_{s s^{\prime}} \delta_{\tau}^{\lambda} \delta_{\mu}^{\nu} \delta_{\beta}^{\alpha} \delta^{(3)}\left(\vec{p}-\overrightarrow{p^{\prime}}\right) .
\end{aligned}
$$

The following relations along many others are established:

$$
\begin{aligned}
& \sum_{\lambda= \pm}<\chi_{\lambda}\left|\underset{\eta}{\hat{\Psi}}(\eta) \underset{\eta}{\overline{\hat{\Psi}}}\left(\eta^{\prime}\right)\right| \chi_{\lambda}>_{0}=-i\left(\eta \eta^{\prime}\right)_{0} \underset{\eta}{G}\left(\eta-\eta^{\prime}\right), \\
& \sum_{\lambda= \pm}<\chi_{\lambda}\left|\underset{u}{\hat{\Psi}}(u) \underset{u}{\hat{\Psi}}\left(u^{\prime}\right)\right| \chi_{\lambda}>_{0}=i\left(u u^{\prime}\right)_{0} \underset{u}{G}\left(u-u^{\prime}\right) \\
& \sum_{\lambda= \pm}<\chi_{\lambda}\left|\hat{\Phi}(\zeta) \overline{\hat{\Phi}}\left(\zeta^{\prime}\right)\right| \chi_{\lambda}>_{0}=-i\left(\zeta \zeta^{\prime}\right)_{0} \underset{\zeta}{G}\left(\zeta-\zeta^{\prime}\right)= \\
& =-i\left[\left(\eta \eta^{\prime}\right)_{0} \underset{\eta}{G}\left(\eta-\eta^{\prime}\right)-\left(u u^{\prime}\right)_{0} \underset{u}{G}\left(u-u^{\prime}\right)\right],
\end{aligned}
$$

where the subscript $(0)\left(<>_{0},()_{0}\right)$ specifies the cone $(x y)_{0}=x_{(+\alpha)} y^{(+\alpha)}=x_{(-\alpha)} y^{(-\alpha)}$. The Green's functions are used

$$
\underset{\eta}{G}\left(\eta-\eta^{\prime}\right)=-(i \underset{\eta}{\hat{\partial}}+m) \underset{\eta}{\Delta}\left(\eta-\eta^{\prime}\right), \quad \underset{u}{G}\left(u-u^{\prime}\right)=-(i \underset{u}{\hat{\partial}}+m) \underset{u}{\Delta}\left(u-u^{\prime}\right)
$$

where the $\underset{\eta}{\Delta}\left(\eta-\eta^{\prime}\right)$ and $\Delta_{u}\left(u-u^{\prime}\right)$ are invariant singular functions. Thus

$$
\begin{aligned}
& \sum_{\lambda= \pm}<\chi_{\lambda}|\hat{\Phi}(\zeta) \overline{\hat{\Phi}}(\zeta)| \chi_{\lambda}>=\sum_{\lambda= \pm}<\chi_{\lambda}|\overline{\hat{\Phi}}(\zeta) \hat{\Phi}(\zeta)| \chi_{\lambda}>= \\
& -i \lim _{\zeta \rightarrow \zeta^{\prime}}\left(\zeta \zeta^{\prime}\right) \underset{\zeta}{G}\left(\zeta-\zeta^{\prime}\right)=-i\left[\lim _{\eta \rightarrow \eta^{\prime}}\left(\eta \eta^{\prime}\right) \underset{\eta}{G}\left(\eta-\eta^{\prime}\right)-\lim _{u \rightarrow u^{\prime}}\left(u u^{\prime}\right) \underset{u}{G}\left(u-u^{\prime}\right)\right] .
\end{aligned}
$$

We may consider so-called "causal" Green's functions $\underset{\eta_{F}}{G} \underset{u_{F}}{G}$ and $\underset{\zeta_{F}}{G}$ for $\eta-, u-$ and $\zeta$-type goyak fields, respectively. Usually they appeared in quantum field theory as an 
expression of "causality", in place of the retarded functions and characterized by the boundary condition that only positive frequency occur for $\eta_{0}>0 \quad\left(u_{0}>0\right)$, only negative for $\eta_{0}<0 \quad\left(u_{0}<0\right)$ :

$$
\left(\zeta \zeta^{\prime}\right)_{0} G_{\zeta_{F}}\left(\zeta-\zeta^{\prime}\right)=\left(\eta \eta^{\prime}\right)_{0} G_{\eta_{F}}\left(\eta-\eta^{\prime}\right)-\left(u u^{\prime}\right)_{0} G_{u_{F}}\left(u-u^{\prime}\right) .
$$

\subsection{Realization of The Manifold $G(2.2 .3)$}

Realization of the manifold $G(2.2 .3)$ is expressed in the milder restriction imposed on the matrix element $\sum_{\lambda= \pm}<\chi_{\lambda}|\hat{\Phi}(\zeta) \overline{\hat{\Phi}}(\zeta)| \chi_{\lambda}>$, which is as a geometric object required to be finite. The latter reads

$$
\sum_{\lambda= \pm}<\chi_{\lambda}|\hat{\Phi}(\zeta) \overline{\hat{\Phi}}(\zeta)| \chi_{\lambda}>=\sum_{\lambda= \pm}<\chi_{\lambda}|\overline{\hat{\Phi}}(\zeta) \hat{\Phi}(\zeta)| \chi_{\lambda}>=\zeta_{\zeta}^{2}{\underset{\zeta}{F}}_{F}(0)<\infty
$$

This requirement should begin to manifest its virtue in the special case, when due to eq.(9.1) the following relations hold for each ordinary goyak:

$$
\begin{aligned}
& \underset{\zeta_{F}}{G}(0)={\underset{\eta}{\eta_{F}}}_{F}(0)=\int \frac{d^{4} p_{\eta}}{(2 \pi)^{4}} \frac{p_{\eta}+m}{p^{2}+i \varepsilon}={\underset{u}{F}}_{F}(0)=\int \frac{d^{4} p_{u}}{(2 \pi)^{4}} \frac{p_{u}+m}{p^{2}+i \varepsilon}=
\end{aligned}
$$

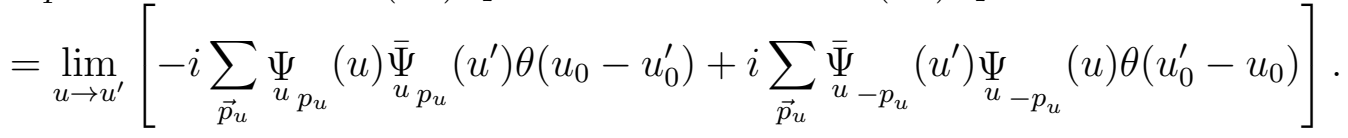

Then satisfying the condition eq.(9.19), the length of each vector $\zeta=e^{(\lambda, \mu, \alpha)} \zeta_{(\lambda, \mu, \alpha)} \in$ $G(2.2 .3)$ (see eq.(9.18)) compulsory should be equaled zero

$$
\zeta^{2}=\eta^{2}-u^{2}=0
$$

Thus, it brings us to the conclusion: the main requirement eq.(9.19) provided by eq.(9.20) yields the realization of the flat manifold $G(2.2 .3)$, which subsequently leads to Minkowski flat space $M^{4}$ (Part I).

$$
G(2.2 .3)=\underset{\eta}{G}(2.3) \oplus \underset{u}{G}(2.3)
$$

The condition eq.(9.21) provides the invariance of line element in $G(2.3)$, as well as in $M^{4}$. In the case of distorted manifold $G(223)$ (sec. 6) [1-5], instead of eq.(9.21) one obviously has

$$
d \zeta^{2}=d \eta^{2}-d u^{2}=0,\left.\quad d \eta^{2}\right|_{6 \rightarrow 4}=d s^{2}=g_{\mu \nu} d x^{\mu} d x^{\nu}=i n v=d u^{2} .
$$

Thus, the principle of Relativity comes into being with the geometry. We have reached this important conclusion, which furnishes the proof of our main idea that the geometry is derivative.

\section{A System of Identical Goyaks}

Next we discuss briefly the quantum theory situation, which corresponds to simultaneously presence of many identical goyak fields. Certainly, if there are $n$ identical goyaks with linkcoordinates $\zeta_{1}, \zeta_{2}, \ldots, \zeta_{n}$ the antisymmetrical state function $\Psi$ will be a function of all of 
them and presents the system of $n$ fermions $\Psi\left(\zeta_{1}, \zeta_{2}, \ldots, \zeta_{n}\right)$, which implies the Fermi-Dirac statistics. To describe the $n$-goyak fermion system by means of quantum field theory, it will be advantageous to make use of convenient method of constructing the state vector of physical system by proceeding from the vacuum state as a very point of origin. One ought to modify the operators eq.(8.7) in order to provide an anticommutation is being valid in both cases acting on the same as well as different states:

$$
\hat{\gamma}_{(\lambda, \mu, \alpha)}^{r} \Rightarrow \hat{\gamma}_{(\lambda, \mu, \alpha)}^{r} \eta_{r}^{\lambda \mu}, \quad \hat{\gamma}_{r}^{(\lambda, \mu, \alpha)} \Rightarrow \eta_{r}^{\lambda \mu} \hat{\gamma}_{r}^{(\lambda, \mu, \alpha)}=\hat{\gamma}_{r}^{(\lambda, \mu, \alpha)} \eta_{r}^{\lambda \mu}, \quad\left(\eta_{r}^{\lambda \mu}\right)^{+}=\eta_{r}^{\lambda \mu},
$$

for fixed $\lambda, \mu, \alpha$, where $\eta_{r}^{\lambda \mu}$ is a diagonal operator in the space of occupation numbers. Therewith, at $r_{i}<r_{j}$ one gets

$$
\hat{\gamma}_{(\lambda, \mu, \alpha)}^{r_{i}} \eta_{r_{j}}^{\lambda \mu}=-\eta_{r_{j}}^{\lambda \mu} \hat{\gamma}_{(\lambda, \mu, \alpha)}^{r_{i}}, \quad \hat{\gamma}_{(\lambda, \mu, \alpha)}^{r_{j}} \eta_{r_{i}}^{\lambda \mu}=\eta_{r_{i}}^{\lambda \mu} \hat{\gamma}_{(\lambda, \mu, \alpha)}^{r_{j}} .
$$

The operators of corresponding occupation numbers, for fixed $\lambda, \mu, \alpha$, are

$$
\hat{N}_{r}^{\lambda \mu}=\hat{\gamma}_{(\lambda, \mu, \alpha)}^{r} \hat{\gamma}_{r}^{(\lambda, \mu, \alpha)}=\hat{N}_{r}^{\lambda} \otimes \hat{N}_{r}^{\mu}
$$

where we make use of $\hat{N}_{r}^{1}=\hat{O}_{1}^{r} \hat{O}_{r}^{1}=\left(\alpha_{1} \alpha_{2}\right)_{r}, \hat{N}_{r}^{2}=\hat{O}_{2}^{r} \hat{O}_{r}^{2}=\left(\alpha_{2} \alpha_{1}\right)_{r}$. As far as diagonal operators $\left(1-2 \hat{N}_{r}^{\lambda \mu}\right)$. anticommute with the $\hat{\gamma}_{(\lambda, \mu, \alpha)}^{r}$, then

$\eta_{r_{i}}^{\lambda \mu}=\prod_{r=1}^{r_{i}-1}\left(1-2 \hat{N}_{r}^{\lambda \mu}\right)$, provided

$$
\eta_{r_{i}}^{11} \Psi\left(n_{1}, \ldots, n_{N} ; 0 ; 0 ; 0\right)=\prod_{r=1}^{r_{i}-1}(-1)^{n_{r}} \Psi\left(n_{1}, \ldots, n_{N} ; 0 ; 0 ; 0\right),
$$

and so on. Here the occupation numbers $n_{r}\left(m_{r}, q_{r}, t_{r}\right)$ are introduced, which refer to the $r$-th states corresponding to operators $\hat{\gamma}_{(1,1, \alpha)}^{r}\left(\hat{\gamma}_{(1,2, \alpha)}^{r}, \hat{\gamma}_{(2,1, \alpha)}^{r}, \hat{\gamma}_{(2,2, \alpha)}^{r}\right)$ either empty $\left(n_{r}, \ldots, t_{r}=0\right)$ or occupied $\left(n_{r}, \ldots, t_{r}=1\right)$. To save writing we abbreviate the modified operators by the same symbols. The creation operator $\hat{\gamma}_{(\lambda, \mu, \alpha)}^{r_{i}}$ by acting on free state $\mid 0>_{r_{i}}$ yields the one-occupied state $\mid 1>_{r_{i}}$ with the phase + or - depending of parity of the number of goyaks in the states $r<r_{i}$. Modified operators satisfy the same anticommutation relations of the operators eq.(8.7). It is convenient to make use of notation $\hat{\gamma}_{r}^{(\lambda, \mu, \alpha)} \equiv e_{r}^{(\lambda, \mu, \alpha)} \hat{b}_{(r \alpha)}^{\lambda \mu}, \quad \hat{\gamma}_{(\lambda, \mu, \alpha)}^{r} \equiv e_{(\lambda, \mu, \alpha)}^{r} \hat{b}_{\lambda \mu}^{(r \alpha)}$, and abbreviate the pair of indices $(r \alpha)$ by the single symbol $r$. Then, non vanishing general anticommutation relations hold

$$
\begin{aligned}
& <1,1\left|\left\{\hat{b}_{r}^{11}, \hat{b}_{11}^{r^{\prime}}\right\}\right| 1,1>=<2,2\left|\left\{\hat{b}_{r}^{22}, \hat{b}_{22}^{r^{\prime}}\right\}\right| 2,2>= \\
& =<1,2\left|\left\{\hat{b}_{r}^{12}, \hat{b}_{12}^{r^{\prime}}\right\}\right| 1,2>=<2,1\left|\left\{\hat{b}_{r}^{21}, \hat{b}_{(21}^{r^{\prime}}\right\}\right| 2,1>=\delta_{r}^{r^{\prime}} .
\end{aligned}
$$

The operator of occupation number in terms of new operators is $\hat{N}_{r}^{\lambda \mu}=\hat{b}_{\lambda \mu}^{r} \hat{b}_{r}^{\lambda \mu}$. The vacuum state reads eq.(8.13), with the normalization requirement

$$
<\chi^{0}\left(\nu_{1}^{\prime}, \nu_{2}^{\prime}, \nu_{3}^{\prime}, \nu_{4}^{\prime}\right) \mid \chi^{0}\left(\nu_{1}, \nu_{2}, \nu_{3}, \nu_{4}\right)>=\prod_{i=1}^{4} \delta_{\nu_{i} \nu_{i}^{\prime}} .
$$

The state vectors

$$
\begin{aligned}
& \chi\left(\left\{n_{r}\right\}_{1}^{N} ;\left\{m_{r}\right\}_{1}^{M} ;\left\{q_{r}\right\}_{1}^{Q} ;\left\{t_{r}\right\}_{1}^{T} ;\left\{\nu_{r}\right\}_{1}^{4}\right)=\left(\hat{b}_{11}^{N}\right)^{n_{N}} \cdots\left(\hat{b}_{11}^{1}\right)^{n_{1}} \cdot \\
& \cdot\left(\hat{b}_{12}^{M}\right)^{m_{M}} \cdots\left(\hat{b}_{12}^{1}\right)^{m_{1}} \cdot\left(\hat{b}_{21}^{Q}\right)^{q_{Q}} \cdots\left(\hat{b}_{21}^{1}\right)^{q_{1}} \cdots\left(\hat{b}_{22}^{T}\right)^{t_{T}} \cdots\left(\hat{b}_{22}^{1}\right)^{t_{1}} \chi^{0}\left(\nu_{1}, \nu_{2}, \nu_{3}, \nu_{4}\right),
\end{aligned}
$$


where $\left\{n_{r}\right\}_{1}^{N}=n_{1}, \ldots, n_{N}$ and so on, are the eigen-functions of modified operators. They form a whole set of orthogonal vectors

$$
\begin{aligned}
& <\chi\left(\left\{n_{r}^{\prime}\right\}_{1}^{N} ;\left\{m_{r}^{\prime}\right\}_{1}^{M} ;\left\{q_{r}^{\prime}\right\}_{1}^{Q} ;\left\{t_{r}^{\prime}\right\}_{1}^{T} ;\left\{\nu_{r}^{\prime}\right\}_{1}^{4}\right) \mid \chi\left(\left\{n_{r}\right\}_{1}^{N} ;\left\{m_{r}\right\}_{1}^{M} ;\left\{q_{r}\right\}_{1}^{Q} ;\left\{t_{r}\right\}_{1}^{T} ;\left\{\nu_{r}\right\}_{1}^{4}\right)>= \\
& =\prod_{r=1}^{N} \delta_{n_{r} n_{r}^{\prime}} \cdot \prod_{r=1}^{M} \delta_{m_{r} m_{r}^{\prime}} \cdot \prod_{r=1}^{Q} \delta_{q_{r} q_{r}^{\prime}} \cdot \prod_{r=1}^{T} \delta_{t_{r} t_{r}^{\prime}} \cdot \prod_{r=1}^{4} \delta_{\nu_{r} \nu_{r}^{\prime}} .
\end{aligned}
$$

Considering an arbitrary superposition

$$
\chi=\sum_{\substack{n_{1}, \ldots, n_{N}=0 \\ m_{1}, \ldots, m_{M}=0 \\ q_{1}, \ldots, q_{Q}=0 \\ t_{1}, \ldots, t_{T}=0}}^{1} c^{\prime}\left(\left\{n_{r}\right\}_{1}^{N} ;\left\{m_{r}\right\}_{1}^{M} ;\left\{q_{r}\right\}_{1}^{Q} ;\left\{t_{r}\right\}_{1}^{T}\right) \chi\left(\left\{n_{r}\right\}_{1}^{N} ;\left\{m_{r}\right\}_{1}^{M} ;\left\{q_{r}\right\}_{1}^{Q} ;\left\{t_{r}\right\}_{1}^{T} ;\left\{\nu_{r}\right\}_{1}^{4}\right),
$$

the coefficients $c^{\prime}$ of expansion are the corresponding amplitudes of probabilities:

$$
<\left.\chi\left|\chi>=\sum_{\substack{n_{1}, \ldots, n_{N}=0 \\ m_{1}, \ldots, m_{M}=0 \\ q_{1}, \ldots, q_{Q}=0 \\ t_{1}, \ldots, t_{T}=0}}^{1}\right| c^{\prime}\left(\left\{n_{r}\right\}_{1}^{N} ;\left\{m_{r}\right\}_{1}^{M} ;\left\{q_{r}\right\}_{1}^{Q} ;\left\{t_{r}\right\}_{1}^{T}\right)\right|^{2}
$$

The non-vanishing matrix elements of operators $\hat{b}_{r_{k}}^{11}$ and $\hat{b}_{11}^{r_{k}}$ read

$$
\begin{aligned}
& <\chi\left(\left\{n_{r}^{\prime}\right\}_{1}^{N} ; 0 ; 0 ; 0 ; 1,0,0,0\right) \mid \hat{b}_{r_{k}}^{11} \chi\left(\left\{n_{r}\right\}_{1}^{N} ; 0 ; 0 ; 0 ; 1,0,0,0\right)>= \\
& =<1,1\left|\hat{b}_{r_{1}^{\prime}}^{11} \cdots \hat{b}_{r_{n}^{\prime}}^{11} \cdot \hat{b}_{r_{k}}^{11} \cdot \hat{b}_{11}^{r_{n}} \cdots \hat{b}_{11}^{r_{1}}\right| 1,1>= \\
& = \begin{cases}(-1)^{n-k} & \text { if } n_{r}=n_{r}^{\prime} \text { for } r \neq r_{k} \text { and } n_{r_{k}}^{\prime}=0 ; n_{r_{k}}=1, \\
0 & \text { otherwise, }\end{cases} \\
& <\chi\left(\left\{n_{r}^{\prime}\right\}_{1}^{N} ; 0 ; 0 ; 0 ; 1,0,0,0\right) \mid \hat{b}_{11}^{r_{k}} \chi\left(\left\{n_{r}\right\}_{1}^{N} ; 0 ; 0 ; 0 ; 1,0,0,0\right)>= \\
& =<1,1\left|\hat{b}_{r_{1}^{\prime}}^{11} \cdots \hat{b}_{r_{n}^{\prime}}^{11} \cdot \hat{b}_{11}^{r_{k}} \cdot \hat{b}_{11}^{r_{n}} \cdots \hat{b}_{11}^{r_{1}}\right| 1,1>= \\
& = \begin{cases}(-1)^{n^{\prime}-k^{\prime}} & \text { if } n_{r}=n_{r}^{\prime} \text { for } r \neq r_{k} \text { and } n_{r_{k}}=0 ; n_{r_{k}}^{\prime}=1, \\
0 & \text { otherwise, }\end{cases}
\end{aligned}
$$

where one denotes $n=\sum_{r=1}^{N} n_{r}, \quad n^{\prime}=\sum_{r=1}^{N} n_{r}^{\prime}$, the $r_{k}$ and $r_{k}^{\prime}$ are $k$-th and $k^{\prime}$-th terms of regulated sets of $\left\{r_{1}, \ldots, r_{n}\right\} \quad\left(r_{1}<r_{2}<\cdots<r_{n}\right)$ and $\left\{r_{1}^{\prime}, \ldots, r_{n}^{\prime}\right\} \quad\left(r_{1}^{\prime}<r_{2}^{\prime}<\cdots<\right.$ $\left.r_{n}^{\prime}\right)$, respectively. Continuing along this line we get a whole set of explicit forms of matrix elements for the rest of operators $\hat{b}_{r_{k}}^{\lambda \mu}$ and $\hat{b}_{\lambda \mu}^{r_{k}}$. There up on

$$
\begin{aligned}
& \sum_{\left\{\nu_{r}\right\}=0}^{1}<\chi^{0}|\hat{\Phi}(\zeta)| \chi>=\sum_{\left\{\nu_{r}\right\}=0}^{1}<\chi^{0}\left|\hat{\gamma}_{r}^{(\lambda, \mu, \alpha)} \Phi_{(\lambda, \mu, \alpha)}^{r}(\zeta)\right| \chi>= \\
& =\sum_{r=1}^{N} c_{n_{r}}^{\prime} e_{n_{r}}^{(1,1, \alpha)} \Phi_{(1,1, \alpha)}^{n_{r}}+\sum_{r=1}^{M} c_{m_{r}}^{\prime} e_{m_{r}}^{(1,2, \alpha)} \Phi_{(1,2, \alpha)}^{m_{r}}+\sum_{r=1}^{Q} c_{q_{r}}^{\prime} e_{q_{r}}^{(2,1, \alpha)} \Phi_{(2,1, \alpha)}^{q_{r}}+\sum_{r=1}^{T} c_{t_{r}}^{\prime} e_{t_{r}}^{(2,2, \alpha)} \Phi_{(2,2, \alpha)}^{t_{r}},
\end{aligned}
$$


provided

$$
\begin{aligned}
& c_{n_{r}}^{\prime} \equiv \delta_{1 n_{r}} c^{\prime}\left(0, \ldots, n_{r}, \ldots, 0 ; 0 ; 0 ; 0\right), \quad c_{m_{r}}^{\prime} \equiv \delta_{1 m_{r}} c^{\prime}\left(0 ; 0, \ldots m_{r}, \ldots, 0 ; 0 ; 0\right) \\
& c_{q_{r}}^{\prime} \equiv \delta_{1 q_{r}} c^{\prime}\left(0 ; 0 ; 0, \ldots q_{r}, \ldots, 0 ; 0\right), \quad c_{t_{r}}^{\prime} \equiv \delta_{1 t_{r}} c^{\prime}\left(0 ; 0 ; 0 ; 0, \ldots t_{r}, \ldots, 0\right)
\end{aligned}
$$

Hereinafter we change the notation to

$$
\begin{aligned}
& \bar{c}\left(r^{11}\right)=c_{n_{r}}^{\prime}, \quad \bar{c}\left(r^{21}\right)=c_{q_{r}}^{\prime}, \quad N_{11}=N, \quad N_{21}=Q, \\
& \bar{c}\left(r^{12}\right)=c_{m_{r}}^{\prime}, \quad \bar{c}\left(r^{22}\right)=c_{t_{r}}^{\prime}, \quad N_{12}=M, \quad N_{22}=T,
\end{aligned}
$$

and make use of

$$
\begin{aligned}
& F_{r^{\lambda \mu}}=\sum_{\alpha} e_{r^{\lambda \mu}}^{(\lambda, \mu, \alpha)} \Phi_{(\lambda, \mu, \alpha)}^{r^{\lambda \mu}}, \quad F^{r^{\lambda \mu}}=\sum_{\alpha} e_{(\lambda, \mu, \alpha)}^{r^{\lambda \mu}} \Phi_{r^{\lambda \mu}}^{(\lambda, \mu, \alpha)}=\bar{F}_{r^{\lambda \mu}}, \\
& \sum_{\left\{\nu_{r}\right\}=0}^{1}<\chi^{0}|\hat{A}| \chi>\equiv<\chi^{0}\|\hat{A}\| \chi>, \quad \sum_{\left\{\nu_{r}\right\}=0}^{1}<\chi|\hat{A}| \chi^{0}>\equiv<\chi\|\hat{A}\| \chi^{0}>.
\end{aligned}
$$

Then, the matrix elements of operator-vector and co-vector fields take the forms

$$
\begin{aligned}
& <\chi^{0}\|\hat{\Phi}(\zeta)\| \chi>=\sum_{\lambda \mu=1}^{2} \sum_{r^{\lambda \mu}=1}^{N_{\lambda \mu}} \bar{c}\left(r^{\lambda \mu}\right) F_{r^{\lambda \mu}}(\zeta), \\
& <\chi\|\bar{\Phi}(\zeta)\| \chi^{0}>=\sum_{\lambda \mu=1}^{2} \sum_{r^{\lambda \mu}=1}^{N_{\lambda \mu}} \bar{c}^{*}\left(r^{\lambda \mu}\right) F^{r^{\lambda \mu}}(\zeta) .
\end{aligned}
$$

Explicitly the matrix element of $n$-operator vector fields, in general, reads

$$
\begin{aligned}
& \frac{1}{\sqrt{n !}}<\chi^{0}\left\|\hat{\Phi}\left(\zeta_{1}\right) \cdots \hat{\Phi}\left(\zeta_{n}\right)\right\| \chi>= \\
& =\frac{1}{\sqrt{n !}}\left\{\sum_{\lambda \mu=1}^{2}\right\}_{1}^{n} \sum_{r_{1}^{\lambda \mu}, \ldots r_{n}^{\lambda \mu}=1}^{N_{\lambda \mu}} \bar{c}\left(r_{1}^{\lambda \mu}, \ldots, r_{n}^{\lambda \mu}\right) \sum_{\sigma \in S_{n}} \operatorname{sgn}(\sigma) F_{r_{1}^{\lambda \mu}}\left(\zeta_{1}\right) \cdots F_{r_{n}^{\lambda \mu}}\left(\zeta_{n}\right)=
\end{aligned}
$$

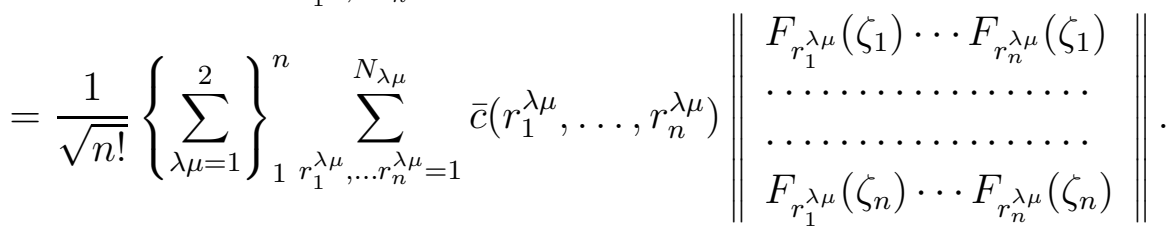

The summation is extended over all permutations of indices $\left(r_{1}^{\lambda \mu}, \ldots, r_{n}^{\lambda \mu}\right)$ of the integers $1,2, \ldots, n$, whereas the antisymmetrical eigen-functions are sums of the same terms with alternating signs in dependence of a parity $\operatorname{sgn}(\sigma)$ of transposition; $\left\{\sum_{\lambda \mu}^{2}\right\}_{1}^{n} \equiv \sum_{\lambda_{1} \mu_{1}}^{2} \ldots \sum_{\lambda_{n} \mu_{n}}^{2}$ and $r_{i}^{\lambda \mu} \equiv r^{\lambda_{i} \mu_{i}}$. Here we make use of

$$
\begin{aligned}
& \bar{c}\left(r_{1}^{11}, \ldots, r_{n}^{11}\right)=c^{\prime}\left(n_{1}, \ldots, n_{N} ; 0 ; 0 ; 0\right), \quad \bar{c}\left(r_{1}^{12}, \ldots, r_{n}^{12}\right)=c^{\prime}\left(0 ; m_{1}, \ldots, m_{M} ; 0 ; 0\right) \\
& \bar{c}\left(r_{1}^{21}, \ldots, r_{n}^{21}\right)=c^{\prime}\left(0 ; 0 ; q_{1}, \ldots, q_{Q} ; 0\right), \quad \bar{c}\left(r_{1}^{22}, \ldots, r_{n}^{22}\right)=c^{\prime}\left(0 ; 0 ; 0 ; t_{1}, \ldots, t_{T}\right)
\end{aligned}
$$


Analogical expression can be obtained for co-vector fields. Following to this procedure one also gets

$$
\begin{aligned}
& \sum_{\lambda=1}^{2}<\chi\left(\left\{n_{r}^{\prime}\right\}_{1}^{N} ; 0 ; 0 ; 0 ; \lambda ; 0\right) \mid \hat{b}_{\eta_{r_{k}}}^{+} \chi\left(\left\{n_{r}\right\}_{1}^{N} ; 0 ; 0 ; 0 ; \lambda ; 0\right)>= \\
& = \begin{cases}(-1)^{n-k} & \text { if } n_{r}=n_{r}^{\prime} \text { for } r \neq r_{k} \text { and } n_{r_{k}}^{\prime}=0 ; n_{r_{k}}=1, \\
0 & \text { otherwise, }\end{cases} \\
& \sum_{\lambda=1}^{2}<\chi\left(\left\{n_{r}^{\prime}\right\}_{1}^{N} ; 0 ; 0 ; 0 ; \lambda ; 0\right) \mid \hat{b}_{\eta_{+}}^{r_{k}} \chi\left(\left\{n_{r}\right\}_{1}^{N} ; 0 ; 0 ; 0 ; \lambda ; 0\right)>= \\
& = \begin{cases}(-1)^{n^{\prime}-k^{\prime}} & \text { if } n_{r}=n_{r}^{\prime} \text { for } r \neq r_{k} \text { and } n_{r_{k}}=0 ; n_{r_{k}}^{\prime}=1, \\
0 & \text { otherwise }\end{cases}
\end{aligned}
$$

and so on. Thus

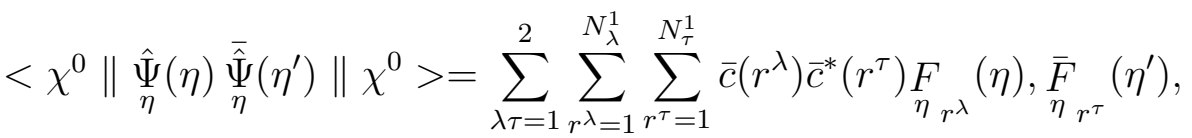

$$
\begin{aligned}
& <\chi^{0}\left\|\underset{u}{\hat{\Psi}}(u) \underset{u}{\overline{\hat{\Psi}}}\left(u^{\prime}\right)\right\| \chi^{0}>=\sum_{\lambda \tau=1}^{2} \sum_{r^{\lambda}=1}^{N_{\lambda}^{2}} \sum_{r^{\tau}=1}^{N_{\tau}^{2}} \bar{c}\left(r^{\lambda}\right) \bar{c}^{*}\left(r^{\tau}\right) \underset{u_{r^{\lambda}}}{F_{i}}(u), \bar{F}_{r^{\tau}}\left(u^{\prime}\right) \text {. }
\end{aligned}
$$

The expression of generalized Green's function $\underset{\eta}{\widetilde{G}}\left(\eta-\eta^{\prime}\right)$ may be written

$$
\widetilde{G}\left(\eta-\eta^{\prime}\right)=\sum_{r^{\lambda}=1}^{N_{\lambda}^{1}}\left|\bar{c}\left(r^{\lambda}\right)\right|^{2} \underset{\eta_{\lambda}}{G^{\lambda}}\left(\eta-\eta^{\prime}\right), \quad \underset{\eta_{\lambda}}{r^{\lambda}}\left(\eta-\eta^{\prime}\right)=-i T\left(\underset{\eta_{\lambda}}{\Psi_{\lambda} r^{\lambda}}(\eta) \underset{\eta_{\lambda}}{\bar{\Psi}^{r^{\lambda}}}\left(\eta^{\prime}\right)\right),
$$

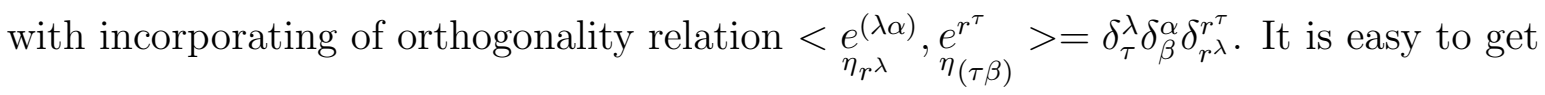

$$
\underset{u}{\widetilde{G}}\left(u-u^{\prime}\right)=\sum_{r^{\lambda}=1}^{N_{\lambda}^{2}}\left|\bar{c}\left(r^{\lambda}\right)\right|^{2} \underset{u_{\lambda}}{r^{\lambda}}\left(u-u^{\prime}\right), \quad \underset{\zeta}{\widetilde{G}}\left(\zeta-\zeta^{\prime}\right)=\sum_{r^{\lambda \mu}=1}^{N_{\lambda \mu}}\left|\bar{c}\left(r^{\lambda \mu}\right)\right|^{2}{\underset{\zeta}{\lambda \mu}}_{r^{\lambda \mu}}\left(\zeta-\zeta^{\prime}\right),
$$

provided

$$
\underset{u_{\lambda}}{G^{r^{\lambda}}}\left(u-u^{\prime}\right)=-i T\left(\underset{u_{\lambda}}{\Psi_{\lambda}^{r^{\lambda}}}(u) \underset{u_{\lambda}}{\bar{\Psi}^{r^{\lambda}}}\left(u^{\prime}\right)\right), \quad \underset{\zeta_{\lambda \mu}}{G^{r^{\lambda \mu}}}\left(\zeta-\zeta^{\prime}\right)=-i T\left(\Phi_{\lambda \mu}^{r^{\lambda \mu}}(\zeta) \bar{\Phi}_{\lambda \mu}^{r^{\lambda \mu}}\left(\zeta^{\prime}\right)\right),
$$

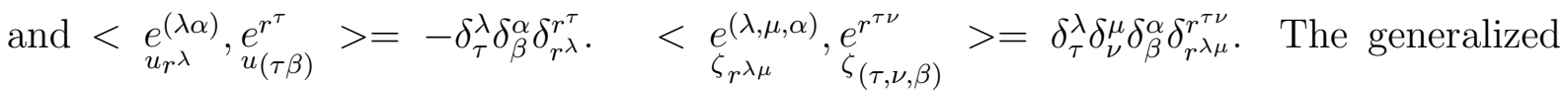
causal Green's functions associate with the fields defined on the wave manifolds $\widetilde{G}(2.2 .3)=$

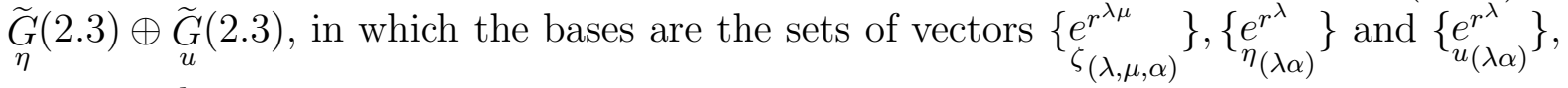
respectively.

\section{Geometric Aspect of Operator Manifold}

The other interesting offshoot of this generalization is a geometric aspect. Here we shall briefly continue the differential geometric treatment of the operator manifold $\hat{G}(2.2 .3)$ and perform a passage from the operator manifold to wave manifold $\widetilde{G}(2.2 .3)$. Let consider, 
for example, the operator-tensor of $(\widehat{n, 0})$-type, which is $\hat{\Phi}\left(\zeta_{1}\right) \otimes \cdots \otimes \hat{\Phi}\left(\zeta_{n}\right)$, where $\otimes$ stands for tensor product. We readily obtain the matrix element of operator-tensor of $(\widehat{n, 0})$-type as follows:

$$
\begin{aligned}
& \frac{1}{\sqrt{n !}}<\chi^{0}\left\|\hat{\Phi}\left(\zeta_{1}\right) \otimes \cdots \otimes \hat{\Phi}\left(\zeta_{n}\right)\right\| \chi>= \\
& =\left\{\sum_{\lambda \mu=1}^{2}\right\}_{1}^{n} \sum_{r_{1}^{\lambda \mu}, \ldots, r_{n}^{\lambda \mu}=1}^{N_{\lambda \mu}} \bar{c}\left(r_{1}^{\lambda \mu}, \ldots, r_{n}^{\lambda \mu}\right) \sum_{\sigma \in S_{n}} \operatorname{sgn}(\sigma) F_{r_{1}^{\lambda \mu}}\left(\zeta_{1}\right) \otimes \cdots \otimes F_{r_{n}^{\lambda \mu}}\left(\zeta_{n}\right)= \\
& =\left\{\sum_{\lambda \mu=1}^{2}\right\}_{1}^{N_{\lambda \mu}} \sum_{r_{1}^{\lambda \mu}, \ldots, r_{n}^{\lambda^{\lambda \mu}}=1}^{n} \bar{c}\left(r_{1}^{\lambda \mu}, \ldots, r_{n}^{\lambda \mu}\right) F_{r_{1}^{\lambda \mu}}\left(\zeta_{1}\right) \wedge \cdots \wedge F_{r_{n}^{\lambda \mu}}\left(\zeta_{n}\right),
\end{aligned}
$$

where $\wedge$ stands for exterior product. It is a straightforward to get also the explicit form of the operator-tensor $(\widehat{0, n})$. The matrix element eq.(11.1) is the geometric objects belonging to manifold $\widetilde{G}(2.2 .3)$, which expose an antisymmetric part of tensor degree. We have reached an important conclusion, that by constructing matrix elements of operatortensors of $\hat{G}(2.2 .3)$ one produces the external products on wave manifold $\widetilde{G}(2.2 .3)$. There up on, the matrix elements of symmetric operator-tensor identically equal zero. To facilitate writing, we abbreviate the set of indices $\left(\lambda_{i}, \mu_{i}, \alpha_{i}\right)$ by the single symbol $i$. The antisymmetric tensor $T_{i_{1} \ldots i_{k}}$ may be defined by single number $T_{1 \ldots k}$. It is convenient to express it in terms of differential operator-forms on $\hat{G}(2.2 .3)$. As the basis in the space of such tensor the operator elements $d \Phi^{i_{1}} \wedge \cdots \wedge d \Phi^{i_{k}}=\sum_{\sigma \in S_{k}} \operatorname{sgn}(\sigma) \hat{\gamma}^{\sigma\left(i_{1}\right.} \otimes \cdots \otimes \hat{\gamma}^{\left.i_{k}\right)}$ are considered, where the summation is extended over all permutations $\left(i_{1}, \ldots, i_{k}\right)$. Corresponding to $T_{i_{1} \ldots i_{k}}$ differential operator-form $\hat{\omega}$ reads $\hat{\omega}=\sum_{i_{1}<\ldots<i_{k}} T_{i_{1} \ldots i_{k}} d \Phi^{i_{1}} \wedge \cdots \wedge d \Phi^{i_{k}}$, the matrix element of which gives rise to

$$
\begin{aligned}
& <\chi^{0}\|\hat{\omega}\| \chi>= \\
& =\sum_{i_{1}<\ldots<i_{k}}\left\{\sum_{\lambda, \mu=1}^{2}\right\}_{1}^{k} \sum_{r_{1}^{\lambda \mu}, \ldots, r_{k}^{\lambda \mu}=1}^{N_{\lambda \mu}} \bar{c}\left(r_{1}^{\lambda \mu}, \ldots, r_{k}^{\lambda \mu}\right) T\left(r_{1}^{\lambda \mu}, \ldots, r_{k}^{\lambda \mu}\right)_{i_{1} \ldots i_{k}} d \Phi_{r_{1}^{\lambda \mu}}^{i_{1}} \wedge \cdots \wedge d \Phi_{r_{k}^{\lambda \mu}}^{i_{k}} .
\end{aligned}
$$

So define the external differential by operator-form $d \hat{\omega}$ of $(k+1)$ degree

$$
d \hat{\omega}=\sum_{\substack{i_{0} \\ i_{1}<\ldots<i_{k}}} \frac{\partial T_{i_{1} \ldots i_{k}}}{\partial \Phi^{i_{0}}} d \Phi^{i_{0}} \wedge d \Phi^{i_{1}} \wedge \cdots \wedge d \Phi^{i_{k}}=\sum_{i_{1}<\ldots<i_{k}}\left(d T_{i_{1} \ldots i_{k}}\right) \wedge d \Phi^{i_{1}} \wedge \cdots \wedge d \Phi^{i_{k}}
$$

then

$$
\begin{aligned}
& <\chi^{0}\|d \hat{\omega}\| \chi>= \\
& =\sum_{i_{1}<\ldots<i_{k}}\left\{\sum_{\lambda, \mu=1}^{2}\right\}_{1}^{k} \sum_{r_{1}^{\lambda \mu}, \ldots, r_{k}^{\lambda \mu}=1}^{N_{\lambda \mu}} \bar{c}\left(r_{1}^{\lambda \mu}, \ldots r_{k}^{\lambda \mu}\right)\left(d T\left(r_{1}^{\lambda \mu}, \ldots, r_{k}^{\lambda \mu}\right)_{i_{1} \ldots i_{k}}\right) \wedge d \Phi_{r_{1}^{\lambda \mu}}^{i_{1}} \wedge \cdots \wedge d \Phi_{r_{k}^{\lambda \mu}}^{i_{k}} .
\end{aligned}
$$

This is the external differential of the corresponding form on wave manifold $\widetilde{G}(2.2 .3)$. Continuing along this line we may employ the analog of integration of the forms. The matrix element of any geometric object of operator manifold $\hat{G}(2.2 .3)$ yields corresponding 
geometric object of wave manifold $\widetilde{G}(2.2 .3)$. Thus, all geometric objects belonging to the latter can be constructed by means of matrix elements of corresponding geometric objects of the operator manifold $\hat{G}(2.2 .3)$.

\section{The Groups $\hat{F}$ and $\widetilde{F}$}

Next we deal with the treatment of principles of the theory of the groups $\hat{F}, \widetilde{F}$ and their representations. They refer to continuous and discrete transformations of symmetries of operator $\hat{G}(2.2 .3)$ and wave $\widetilde{G}(2.2 .3)$ manifolds, respectively. The action of linear operator $\hat{F}$ upon any operator-vector of $\hat{G}(2.2 .3)$ is completely defined by its action upon the operator basis $\{\hat{\gamma}\}$. We consider the homogeneous $\hat{F}$ transformations of basis vectors eq. $(8.7)$

$$
{\hat{\gamma^{\prime}}}^{(\lambda, \mu, \alpha)}=\hat{F}_{(\tau, \nu, \beta)}^{(\lambda, \mu, \alpha)} \hat{\gamma}^{(\tau, \nu, \beta)}, \quad{\hat{\gamma^{\prime}}}_{(\lambda, \mu, \alpha)}=\hat{\gamma}_{(\tau, \nu, \beta)} \hat{F}_{(\lambda, \mu, \alpha)}^{(\tau, \nu, \beta)}, \quad\left(\hat{\gamma}^{\prime}=\hat{F} \hat{\gamma}, \quad \overline{\hat{\gamma}}^{\prime}=\overline{\hat{\gamma}} \overline{\hat{F}}^{T}\right)
$$

provided by $\hat{F}_{(\tau, \nu, \beta)}^{(\lambda, \mu, \alpha)}$, which is a matrix operator with respect to basis $\{\hat{\gamma}\}$, and $\hat{F} \hat{F}^{T}=$ $\hat{F}^{T} \hat{F}=\hat{\delta}$. The $\hat{\delta}$ is called the operator Kronecker symbol. It is being fashioned after the conventional symbol and best visualized as $<\chi^{0}\left\|\hat{\delta}_{(\rho, \omega, \gamma)}^{(\tau, \nu, \beta)}\right\| \chi^{0}>=\delta_{(\rho, \omega, \gamma)}^{(\tau, \nu, \beta)}=\delta_{\rho}^{\tau} \delta_{\omega}^{\nu} \delta_{\gamma}^{\beta}$. The transformations eq.(12.1) leave unchanged the matrix element of the norm of operatorvector. The unification of homogeneous $\hat{F}$-transformations with 12-dimensional operatortransfers has reflected the properties of continuous symmetries of operator manifold $\hat{G}(2.2 .3)$. Meanwhile the matrix elements of the constant operator 12-vectors $\hat{a} \in \hat{G}(2.2 .3)$ yield the constant 12 -vectors $a \in G(2.2 .3)$.

The matrix element of eq.(12.1) yields corresponding transformations of homogeneous wave group $\widetilde{F}$

$$
\begin{aligned}
& <\chi^{0}\left\|\hat{\gamma}^{\prime}\right\| \chi>=<\chi^{0}\|\hat{F}\| \chi^{0}><\chi^{0}\|\hat{\gamma}\| \chi>=\widetilde{F}<\chi^{0}\|\hat{\gamma}\| \chi>, \\
& <\chi\left\|\hat{\bar{\gamma}}^{\prime}\right\| \chi^{0}>=<\chi\|\hat{\hat{\gamma}}\| \chi^{0}><\chi^{0}\left\|\hat{F}^{T}\right\| \chi^{0}>=<\chi\|\hat{\gamma}\| \chi^{0}>\widetilde{F}^{T} .
\end{aligned}
$$

It reads in component form

$$
\begin{aligned}
& \widetilde{F}_{(\tau, \nu, \beta)}^{(\lambda, \mu, \alpha)}=\left(\sum_{r^{\tau \nu}=1}^{N_{\tau \nu}} \bar{c}\left(r^{\tau \nu}\right)\right)^{-1} \sum_{r^{\lambda \mu}=1}^{N_{\lambda \mu}} \bar{c}\left(r^{\lambda \mu}\right) K_{(\tau, \nu, \beta)}^{(\lambda, \mu, \alpha)}\left(r^{\lambda \mu}, r^{\tau \nu}\right), \\
& \widetilde{F}_{(\lambda, \mu, \alpha)}^{(\tau, \nu, \beta)}=\left(\sum_{r^{\tau \nu}=1}^{N_{\tau \nu}} \bar{c}^{*}\left(r^{\tau \nu}\right)\right)^{-1} \sum_{r^{\lambda \mu}=1}^{N_{\lambda \mu}} \bar{c}^{*}\left(r^{\lambda \mu}\right) K_{(\lambda, \mu, \alpha)}^{(\tau, \nu, \beta)}\left(r^{\tau \nu}, r^{\lambda \mu}\right),
\end{aligned}
$$

provided

$$
\begin{aligned}
& K_{(\tau, \mu, \beta)}^{(\lambda, \mu)}\left(r^{\lambda \mu}, r^{\tau \nu}\right)=<e_{r^{\lambda \mu}}^{(\lambda, \mu, \alpha)}, e_{(\tau, \nu, \beta)}^{r^{\tau \nu}}> \\
& K_{(\lambda, \mu, \alpha)}^{(\tau, \nu, \beta)}\left(r^{\tau \nu}, r^{\lambda \mu}\right)=\left(K^{T}\right)_{(\lambda, \mu, \alpha)}^{(\tau, \nu, \beta)}=<e_{r^{\tau \nu}}^{(\tau, \nu, \beta)}, e_{(\lambda, \mu, \alpha)}^{r^{\lambda \mu}}>
\end{aligned}
$$

which are known as the elements of homogeneous $\hat{K}$ group of continuous symmetries of the manifold $G(2.2 .3)$ [1-5]. Any homogeneous $\hat{K}$-transformation is defined by 12 independent parameters, and the $12 \times 12$-dimensional matrix $\hat{K}$ is orthogonal and unimodal. In general the group $\hat{K}$ is decomposed into 8 components of connectedness $K_{+}^{(++)}, K_{-}^{(++)}$, $K_{+}^{(+-)}, K_{-}^{(+-)}, K_{+}^{(-+)}, K_{-}^{(-+)}, K_{+}^{(--)}, K_{-}^{(--)}$. The sings $(+)$and $(-)$in the parentheses refer respectively to orthochronous and non-orthochronous transformations, but the subscripts 
+ and - specify the special $(|K|=1)$ and non-special $(|K|=-1)$ transformations. According to it, the operator group $\hat{F}$ and wave group $\widetilde{F}$ are decomposed into 8 components of connectedness, respectively $\hat{F}_{+}^{(++)}, \hat{F}_{-}^{(++)}, \hat{F}_{+}^{(+-)}, \hat{F}_{-}^{(+-)}, \hat{F}_{+}^{(-+)}, \hat{F}_{-}^{(-+)}, \hat{F}_{+}^{(--)}, \hat{F}_{-}^{(--)}$; $\widetilde{F}_{+}^{(++)}, \widetilde{F}_{-}^{(++)}, \widetilde{F}_{+}^{(+-)}, \widetilde{F}_{-}^{(+-)}, \widetilde{F}_{+}^{(-+)}, \widetilde{F}_{-}^{(-+)}, \widetilde{F}_{+}^{(--)}, \widetilde{F}_{-}^{(--)}$. In particular, when the matrix K runs upon the homogeneous special group $\widehat{S O}(6.6)$, hence corresponding matrices $\hat{F}$ and $\widetilde{F}$ run upon the homogeneous special groups $\widehat{S O}(6.6)_{\hat{F}}$ and $\widehat{S O}(6.6)_{\widetilde{F}}$.

Alongside with continuous transformations some discrete transformations can be distinguished:

1) The positive co-contra transformation $\hat{P}_{+}$is given by

$$
\left(\hat{\gamma}^{(\lambda, \mu, \alpha)}\right)=\hat{P}_{+}\left(\hat{\gamma}_{(\lambda, \mu, \alpha)}\right)=\left(\hat{\gamma}^{(\lambda, \mu, \alpha)}\right), \quad\left(\hat{\gamma}_{(\lambda, \mu, \alpha)}^{\prime}\right)=\left(\hat{\gamma}_{(\lambda, \mu, \alpha)}\right) \hat{P}_{+}^{T}=\left(\hat{\gamma}_{(\lambda, \mu, \alpha)}\right),
$$

provided $\hat{P}_{+} \hat{P}_{+}^{T}=\hat{P}_{+}^{2}=1$.

2) The negative co-contra transformation $\hat{P}_{-}$is defined as $\hat{P}_{-}=-\hat{P}_{+}$.

3) The total co-co or contra-contra transformations $\hat{P}_{+-}$is in the form $\hat{P}_{+-}=\hat{P}_{+} \hat{P}_{-}=-1$. The matrix elements yield corresponding diagonal discrete transformations of wave group $\widetilde{P}$ :

$$
\begin{aligned}
& \left(\widetilde{P}_{ \pm}\right)_{(\lambda, \mu, \alpha)}^{(\lambda, \mu, \alpha)}=<\chi^{0}\left\|\left(\hat{P}_{ \pm}\right)_{(\lambda, \mu, \alpha)}^{(\lambda, \mu, \alpha)}\right\| \chi^{0}>=\left(\sum_{r^{\lambda \mu}=1}^{N_{\lambda \mu}} \bar{c}^{*}\left(r^{\lambda \mu}\right)\right)^{-1} \sum_{r^{\lambda \mu}=1}^{N_{\lambda \mu}} \bar{c}\left(r^{\lambda \mu}\right)\left(I_{ \pm}\right)_{(\lambda, \mu, \alpha)}^{(\lambda, \mu, \alpha)}\left(r^{\lambda \mu}\right), \\
& \left(\widetilde{P}_{ \pm}\right)_{(\lambda, \mu, \alpha)}^{T(\lambda, \mu, \alpha)}=<\chi^{0}\left\|\left(\hat{P}_{ \pm}\right)_{(\lambda, \mu, \alpha)}^{T(\lambda, \mu, \alpha)}\right\| \chi^{0}>=\left(\sum_{r^{\lambda \mu}=1}^{N_{\lambda \mu}} \bar{c}\left(r^{\lambda \mu}\right)\right)^{-1} \sum_{r^{\lambda \mu}=1}^{N_{\lambda \mu}} \bar{c}^{*}\left(r^{\lambda \mu}\right)\left(I_{ \pm}^{*}\right)_{(\lambda, \mu, \alpha)}^{(\lambda, \mu, \alpha)}\left(r^{\lambda \mu}\right),
\end{aligned}
$$

where $\left(I_{ \pm}\right)_{(\lambda, \mu, \alpha)}^{(\lambda, \mu, \alpha)}\left(r^{\lambda \mu}\right)=<e^{(\lambda, \mu, \alpha)}, e_{(\lambda, \mu, \alpha)}>$ are the elements of the group $I$ of discrete transformations in $G(2.2 .3)$. Therewith $I_{+}$is the particular special orthochron-orthochronous $K_{+}^{(++)}$transformation

$$
\begin{aligned}
& I_{+}{ }^{T}=I_{+}{ }^{*}=\left(I_{+}\right)^{+}, \quad\left(I_{+}\right)^{2}=1, \quad\left|I_{+}\right|=1, \\
& \left(I_{+}\right)_{(1,1, \alpha)}^{(2,1, \alpha)}=\left(I_{+}\right)_{(2,2, \alpha)}^{(1,2, \alpha)}=0, \quad\left(I_{+}\right)_{(1,1, \alpha)}^{(1,1, \alpha)}=\left(I_{+}\right)_{(2,2, \alpha)}^{(2,2, \alpha)}=1 .
\end{aligned}
$$

Accordingly $I_{-} \in K_{-}^{(--)}, \quad I_{+-} \in K_{-}^{(--)}$. It follows that $\hat{P}_{+} \in \hat{F}_{+}^{(++)}, \quad \hat{P}_{-} \in \hat{F}_{-}^{(--)}, \quad \hat{P}_{+-} \in$ $\hat{F}_{-}^{(--)}, \widetilde{P}_{+} \in \widetilde{F}_{+}^{(++)}, \quad \widetilde{P}_{-} \in \widetilde{F}_{-}^{(--)}, \quad \widetilde{P}_{+-} \in \widetilde{F}_{-}^{(--)}$. Thus the group of discrete transformations $\hat{P}$ and $\widetilde{P}$ are consisted respectively of identical $\hat{E} ; \widetilde{E}$ and $\hat{P}_{+}, \hat{P}_{-}, \hat{P}_{+-} ; \widetilde{P}_{+}, \widetilde{P}_{-}, \widetilde{P}_{+-}$ transformations. Each of the groups $\hat{P}$ and $\widetilde{P}$ have only four single-valued irreducible representations, which are all one-dimensional. The important property of Lie algebra of the groups $\widehat{S O}(6.6)_{\hat{F}}$ and $\widehat{S O}(6.6)_{\widetilde{F}}$ can be revealed. The infinitesimal generators of $\widehat{S O}(6.6)_{\widetilde{F}}$, which are the vacuum expectation of corresponding generators of $\widehat{S O}(6.6)_{\hat{F}}$, subsequently may be reduced to independent vectors satisfying the commutation relations of ordinary three-dimensional rotation group $S O(3)_{ \pm}$. Actually, in accordance with eq.(12.3), the infinitesimal generators $\left(\underset{i_{\alpha}}{\hat{h}_{i}}, \hat{H}_{\alpha}\right) \quad(i=\eta, u ; \alpha=1,2,3)$ of the group $\widehat{S O}(6.6)_{\hat{F}}$ and $\left(\underset{i_{\alpha}}{\widetilde{h}}, \widetilde{i_{\alpha}}\right)$ of the group $\widehat{S O}(6.6)_{\widetilde{F}}$ take the form

$$
\begin{aligned}
& \widetilde{h}_{i \alpha}=\underset{i}{e} \otimes \widetilde{h}_{\alpha}=<\chi^{0}\left\|\hat{h}_{i \alpha}\right\| \chi^{0}>=\underset{i}{e} \otimes<\chi^{0}\left\|\hat{h}_{\alpha}\right\| \chi^{0}>\text {, } \\
& \underset{i_{\alpha}}{\vec{H}}=\underset{i}{e} \otimes \widetilde{H}_{\alpha}=<\chi^{0}\left\|\stackrel{\hat{H}}{\alpha}_{\alpha}\right\| \chi^{0}>={\underset{i}{i}}_{e} \otimes<\chi^{0}\left\|\hat{H}_{\alpha}\right\| \chi^{0}>\text {, }
\end{aligned}
$$


at $r^{-}=r^{+} \equiv r$ provided

$$
\begin{aligned}
& \widetilde{h}_{\alpha}=<\chi^{0}\left\|\hat{h}_{\alpha}\right\| \chi^{0}>=<\chi^{0}\left\|i \hat{H}^{[(-\alpha)(+\alpha)]}\right\| \chi^{0}>= \\
& =i\left(\sum_{r=1}^{N} \bar{c}(r)\right)^{-1}\left(\sum_{r=1}^{N} \bar{c}(r)\right) H^{[(-\alpha)(+\alpha)]}=i H^{[(-\alpha)(+\alpha)]}=h_{\alpha}, \\
& \widetilde{H}_{\alpha}=<\chi^{0}\left\|\hat{H}_{\alpha}\right\| \chi^{0}>=H_{\alpha}, \quad \underset{\eta}{e}=\frac{1}{2}\left(\begin{array}{cc}
1 & 1 \\
1 & 1
\end{array}\right), \quad \underset{u}{e}=\frac{1}{2}\left(\begin{array}{cc}
1 & -1 \\
1 & -1
\end{array}\right) .
\end{aligned}
$$

Here the $h_{\alpha}$ and $H_{\alpha} \quad(\alpha=1,2,3)$ are the generators of the group $\widehat{S O}(3.3)$. As far as the generators of the group $\widehat{S O}(6.6)$ obey the transpositional relations of the angular momenta, then the generators of the groups $\widehat{S O}(6.6)_{\hat{F}}$ and $\widehat{S O}(6.6)_{\widetilde{F}}$ have undergone the same reduction. Due to it, the irreducible representation $\hat{D}\left(p_{1}, q_{1} ; p_{2}, q_{2}\right)$ of the group $\widehat{S O}(6.6)_{\hat{F}}$, which can be treated as the reducible representation $\hat{D}\left(p_{1}\right) \otimes \hat{D}\left(q_{1}\right) \otimes \hat{D}\left(p_{2}\right) \otimes$ $\hat{D}\left(q_{2}\right)$ of ordinary three-dimensional rotation group $S O(3)$, is decomposed into irreducible representation of the group $S O(3)$.

\section{Part III. Distorted Goyaks}

\section{The Principle of Identity of Regular Goyaks}

Next we extend the scopes of treatment by considering the processes involving also distorted goyaks. We start with formulation of the principle of identity of regular goyaks, the main idea of which comes to the following: since a generation of each regular goyak in the lowest state $\left(s_{0}\right)$ is the result of its transition into that state from the arbitrary states $\left(s, s^{\prime}, \ldots\right)$, where the goyaks are assumed to be distorted, then all ordinary regular goyaks of u-type are identical structures. Realizing a distortion of the basis $\left\{e_{(\lambda \alpha)}=O_{\lambda} \otimes \sigma_{\alpha}\right\}$, the set of matrices $\{D(\theta)=C(b) \otimes R(\theta)\}$ are the elements of local distortion group (sec. $5,6)$. This principle regarding the system of regular goyaks, which is formed in the lowest state $\left(s_{0}\right)$, holds only for the ordinary goyaks of $u$-type. It is due to the fact that in this system there is only one goyak of $\eta$-type (fundamental) and infinite number of $u$-type goyaks (ordinary). There are a number of advantages to the suggestion of the principle of identity of regular goyaks. It is impressive to see later on that this principle, underlies the most important Gauge principle and the concepts of unitary groups associating with the internal symmetries of elementary particles.

\section{The Distorted Ordinary Goyak's Function}

Distorted ordinary goyak is described by means of link-function

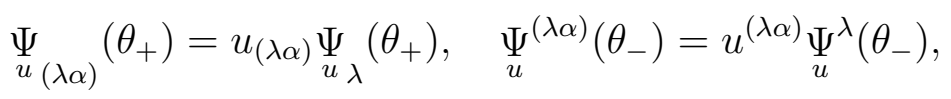

where $u_{(\lambda \alpha)}$ are distorted link-coordinates. We take for granted that the distortion transformations right through the angles $\theta_{+}\left(\theta_{(+k)}, \quad k=1,2,3\right)$ and $\theta_{-}\left(\theta_{(-k)}\right)$ hold for goyaks $\left(\underset{u_{\lambda}}{\Psi}\left(\theta_{+}\right)\right)$and anti-goyaks $\left(\underset{u}{\Psi}\left(\theta_{-}\right)\right)$, respectively. The function $\underset{u}{\Psi}(\theta)=\left(\underset{u_{\lambda}}{\Psi}\left(\theta_{+}\right),{\underset{u}{\Psi}}^{\lambda}\left(\theta_{-}\right)\right)$ 
as the bi-spinor Fermi field, can be derived from Dirac's wave equation of distorted goyak (anti-goyak)(see appendix)

$$
\begin{aligned}
& \left.\left[\underset{u}{i g^{(\lambda \alpha)}}(\theta) \underset{u(\lambda \alpha)}{\partial_{u}}-\Gamma_{u(\lambda \alpha)}(\theta)\right)-m\right] \underset{u}{\Psi}(\theta)=0 \\
& \underset{u}{\Psi}(\theta)\left[i\left(\overleftarrow{\partial}_{(\lambda \alpha)}^{\overleftarrow{\partial_{(\lambda)}}}-\bar{\Gamma}_{u(\lambda \alpha)}(\theta)\right) \underset{u}{g^{(\lambda \alpha)}}(\theta)-m\right]=0
\end{aligned}
$$

where $g_{u}^{(\lambda \alpha)}(\theta)=\underset{u}{V_{(\tau \beta)}^{(\lambda \alpha)}}(\theta) \gamma_{0}^{(\tau \beta)}, \quad \gamma_{0}^{( \pm \alpha)}=\frac{1}{\sqrt{2}}\left(\gamma^{0} \sigma^{\alpha} \pm \gamma^{\alpha}\right), \gamma^{0}, \gamma^{\alpha}$, as usual, are Dirac's matrices. The quantities $\Gamma_{u(\lambda \alpha)}(\theta),\left(\bar{\Gamma}_{u(\lambda \alpha)}(\theta)\right)$ are expressed by Ricci rotation coefficients. To save writing, henceforth within this section we abbreviate the indices $(\lambda \alpha)$ by the single symbol $\mu$, and Latin indices $(i m) \quad(i= \pm, m=1,2,3)$ by $i$. Also we denote $\underset{u_{\mu}}{p} \equiv p_{\mu}, \quad \Gamma_{u_{\mu}} \equiv \Gamma_{\mu}, \quad \underset{u}{\Psi} \equiv \Psi, \quad \underset{u}{g} \equiv \gamma$. Then the equations (14.2) give rise to

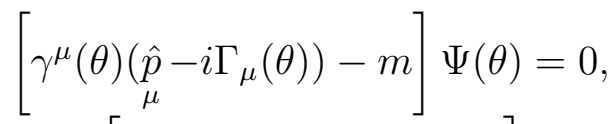

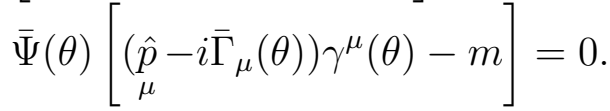

In order to solve eq.(14.3), it is advantageous to transform it into the following one

$$
\left\{-\partial^{2}-m^{2}-(\gamma \Gamma)^{2}+2(\Gamma \gamma)+(\gamma \partial)(\gamma \Gamma)\right\} \Psi=0
$$

provided

$$
\begin{aligned}
& g^{\mu \nu}\left(\hat{p}_{\mu}-i \Gamma_{\mu}\right)\left(\hat{p}_{\nu}-i \Gamma_{\nu}\right)=-\partial^{2}-\Gamma^{2}+2(\Gamma \partial)+(\partial \Gamma), \\
& \frac{1}{2} \sigma^{\mu \nu} F_{\mu \nu}=(\gamma \partial)(\gamma \Gamma)-(\partial \Gamma), \quad(\gamma \partial)(\gamma \Gamma)=\gamma^{\mu} \gamma^{\nu} \partial_{\mu} \Gamma_{\nu}, \\
& \frac{1}{2} \sigma^{\mu \nu}\left[\Gamma_{\mu}, \Gamma_{\nu}\right]=(\gamma \Gamma)^{2}-\Gamma^{2}, \quad \partial^{2}=\partial^{\mu} \partial_{\mu}, \quad \Gamma^{2}=\Gamma^{\mu} \Gamma_{\mu}, \\
& \gamma^{\mu} \gamma^{\nu}=g^{\mu \nu}+\sigma^{\mu \nu}, \quad 2 g^{\mu \nu}=\left\{\gamma^{\mu}, \gamma^{\nu}\right\}, \quad 2 \sigma^{\mu \nu}=\left[\gamma^{\mu}, \gamma^{\nu}\right], \quad \underset{\mu \nu}{F}=\partial_{\mu} \Gamma_{\nu}-\partial_{\nu} \Gamma_{\mu} .
\end{aligned}
$$

We are looking for the solution given in the form $\Psi=e^{-i p u} F(\varphi)$, where $p_{\mu}$ is a constant six-vector $p u=p_{\mu} u_{\mu}$. Next we take for granted that the field of distortion is switched on at $u_{0}=-\infty$ infinitely slowly. Then the function $\Psi$ must match onto the wave function of ordinary regular goyak. Smoothness of the match requires that the numbers $p_{\mu}$ become the components of link-momentum of regular goyak and satisfy the boundary condition $p_{\mu} p_{\mu}=m^{2}=p_{\eta}^{2}$. Due to this requirement, we cancel unwanted solutions, and also clear up the normalization of wave functions

$$
\int \Psi_{p^{\prime}}^{*} \Psi_{p} d^{3} u=\int \bar{\Psi}_{p^{\prime}} \gamma^{0} \Psi_{p} d^{3} u=(2 \pi)^{3} \delta\left(\overrightarrow{p^{\prime}}-\vec{p}\right) .
$$

At $\sqrt{-g} \neq 1$ the gradient of the function $\varphi$ takes the form $\partial_{\mu} \varphi=V_{\mu}^{i} k_{i}, \quad \partial^{\mu} \varphi=V_{i}^{\mu} k^{i}$, where $k_{i}$ are arbitrary constant numbers satisfying the condition $k_{i} k_{i}=0$. The $V_{\mu}^{i}\left(V_{i}^{\mu}\right)$ are congruence parameters of curves (Latin indices refer to tetrad components). Thus $V_{i}^{\mu} V_{\mu}^{j}=g^{\mu \nu} V_{\nu i} V_{\mu}^{j}=\left(g_{0}\right)_{i}^{j}=\delta_{i}^{j}$ and $\partial^{\mu} \varphi \partial_{\mu} \varphi=\left(V_{i}^{\mu} V_{\mu}^{j}\right) k^{i} k_{j}=0$. A substitution in 
eq.(14.4) gives rise to $F^{\prime}=A(\theta) F$, where $(\cdots)^{\prime}$ stands for the derivative with respect to $\varphi$, and

$$
\begin{gathered}
A(\theta)=\frac{2 i(p \Gamma)+m^{2}-p^{2}+(\gamma \Gamma)^{2}-(\gamma \partial)(\gamma \Gamma)}{2 i(k V p)-(k D V)} ; \\
(k V p)=k^{i} V_{i}^{\mu} p_{\mu}, \quad(k D V)=k^{i} D_{\mu} V_{i}^{\mu}, \quad D_{\mu}=\partial_{\mu}-2 \Gamma_{\mu}, \\
p^{2}=p^{\mu} p_{\mu}=g^{\mu \nu}(\theta) p_{\mu} p_{\nu} \quad(k V d u)=k_{i} V_{\mu}^{i} d u^{\mu} .
\end{gathered}
$$

Here we are interested in the right-handed eigen-vectors $F_{r} \quad(r=1,2,3,4)$ corresponding to eigen-values $\mu_{r}$ of matrix $A: A F_{r}=\mu_{r} F_{r}$. They are the roots of polynomial characteristic equation $c(\mu)=\|(\mu I-A)\|=0$. Thus, one gets $F_{r}^{\prime}=\mu_{r} F_{r}$. We may think of the function $F$ as being the product $F=\prod_{r=1}^{4} F_{r}$, hence $(\ln F)^{\prime}=\sum_{r=1}^{4} \mu_{r}=\operatorname{tr} A$, where the trace of matrix $A$ is denoted by $\operatorname{tr} A$. Then $(\ln F)^{\prime}=X_{R}(\theta)-i X_{J}(\theta)$, provided

$$
\begin{aligned}
& X_{R}(\theta)=\operatorname{tr} A_{R}(\theta)=\operatorname{tr}\left\{\frac{-(k D V)\left[m^{2}-p^{2}+(\gamma \Gamma)^{2}-(\gamma \partial)(\gamma \Gamma)\right]+4(k V p)(p \Gamma)}{(k D V)^{2}+4(k V p)^{2}}\right\}, \\
& X_{J}(\theta)=\operatorname{tr} A_{J}(\theta)=2 \operatorname{tr}\left\{\frac{(k V p)\left[m^{2}-p^{2}+(\gamma \Gamma)^{2}-(\gamma \partial)(\gamma \Gamma)\right]+(k D V)(p \Gamma)}{(k D V)^{2}+4(k V p)^{2}}\right\} .
\end{aligned}
$$

One infers at once that the solution clearly is

$$
F(\theta)=C\left(\frac{m}{E_{u}}\right)^{1 / 2} u \exp \left\{\chi_{R}(\theta)-i \chi_{J}(\theta)\right\},
$$

where $C$ is a normalization constant, $u$ is a constant bi-spinor, and

$$
\chi_{R}(\theta)=\int_{0}^{u^{\mu}}(k V d u) X_{R}(\theta), \quad \chi_{J}(\theta)=\int_{0}^{u^{\mu}}(k V d u) X_{J}(\theta) .
$$

So define

$$
\Psi(\theta) \Rightarrow \underset{u_{\lambda}}{\Psi}\left(\theta_{+k}\right)=\left.\bar{u}^{\lambda}\left(\theta_{-k}\right)\right|_{\theta_{-k}=\theta_{+k}}, \quad \bar{\Psi}(\theta) \Rightarrow \underset{u_{\lambda}}{\bar{\Psi}}\left(\theta_{+k}\right)=\left.\underset{u}{\Psi}\left(\theta_{-k}\right)\right|_{\theta_{-k}=\theta_{+k}},
$$

then

$$
\underset{u_{\lambda}}{\Psi}\left(\theta_{+k}\right)=F\left(\theta_{+k}\right) e^{-i p u}=f_{(+)}\left(\theta_{+k}\right) \underset{u \lambda}{\Psi}, \quad \underset{u}{\Psi} \Psi^{\lambda}\left(\theta_{-k}\right)=\underset{u}{\Psi} f_{(-)}\left(\theta_{-k}\right),
$$

provided by the transformation functions

$$
\begin{aligned}
& f_{(+)}\left(\theta_{+k}\right)=C e^{\chi_{R}\left(\theta_{+k}\right)-i \chi_{J}\left(\theta_{+k}\right)}, \\
& f_{(-)}\left(\theta_{-k}\right)=\left.f_{(+)}^{*}\left(\theta_{+k}\right)\right|_{\theta_{+k}=\theta_{-k}}=C e^{\chi_{R}\left(\theta_{-k}\right)+i \chi_{J}\left(\theta_{-k}\right)} .
\end{aligned}
$$

The $\underset{u_{\lambda}}{\Psi}\left(\underset{u}{\Psi^{\lambda}}\right)$ is the plane wave function of regular ordinary goyak. It was assumed that constant bi-spinor $u$ coincides with the amplitude of plane wave, just because of requirement of boundary smoothness, that $\underset{u}{\Psi} \Psi^{J}(\theta)$ must match onto the wave function of ordinary regular goyak at $u_{0}=-\infty$. The inverse transformations at fixed $(k)$ are given by

$$
\underset{u_{\lambda}}{\Psi}=\underset{(+)}{f_{(+)}^{-1}}\left(\theta_{+k}\right) \underset{u_{\lambda}}{\Psi}\left(\theta_{+k}\right), \quad \underset{u}{\Psi} \Psi_{u}^{\lambda}=\underset{\Psi^{\lambda}}{\Psi}\left(\theta_{-k}\right) f_{(-)}^{-1}\left(\theta_{-k}\right) .
$$




\section{Quarks and Color Confinement Principle}

Until now we agreed to consider only a special stable system of regular goyaks, which comes into being in the lowest state $\left(s_{0}\right)$. As it was seen, it consists of one $\eta$-type goyak and infinite number of $u$-type goyaks. They realize the geometry $G(2.2 .3)$ by satisfying the necessary requirements. But such kind of realization is a trivial one, which gives rise to geometry without particles and interactions. However, there is still an other a quite different choice of realization of geometry, which subsequently leads to geometry $G(2.2 .3)$, with the particles and interactions, by the following recipe: Everything said in section 7 will then remain valid provided we make the simple changes. We admit, that the distorted ordinary goyaks have took participation in the realization of geometry instead of regular ordinary goyaks. The ordinary laws regarding these changes apply that we must make use of localized wave packets of distorted ordinary goyaks

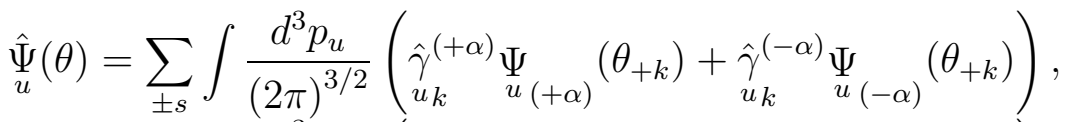

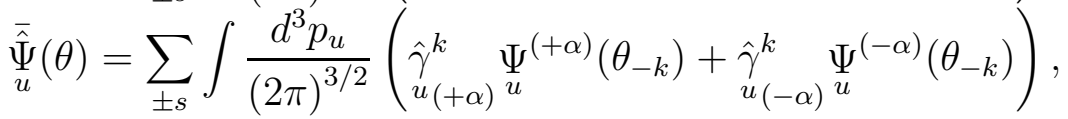

where as usual the summation is extended over all dummy indices, and

$$
\begin{array}{ll}
\underset{u}{\Psi}\left(\theta_{+k}\right)=u_{(+\alpha)} \underset{u+}{\Psi}\left(\theta_{+k}\right), & \underset{u}{\Psi}\left(\theta_{+k}\right)=u_{(-\alpha)} \underset{u}{\Psi}\left(\theta_{+k}\right) \\
{\underset{u}{\Psi}}^{(+\alpha)}\left(\theta_{-k}\right)=u^{(+\alpha)} \underset{u}{\Psi^{+}}\left(\theta_{-k}\right), & {\underset{u}{\Psi}}^{(-\alpha)}\left(\theta_{-k}\right)=u^{(-\alpha)} \underset{u^{-}}{\Psi}\left(\theta_{-k}\right) .
\end{array}
$$

The matrix elements of anti-commutators of generalized expansion coefficients take the form

$$
\begin{aligned}
& <\chi_{-}\left|\left\{{\underset{u}{u_{k}}}_{\hat{\gamma}^{(+\alpha)}}(p, s), \hat{\gamma}_{u_{(+\beta)}}^{k^{\prime}}\left(p^{\prime}, s^{\prime}\right)\right\}\right| \chi_{-}>=
\end{aligned}
$$

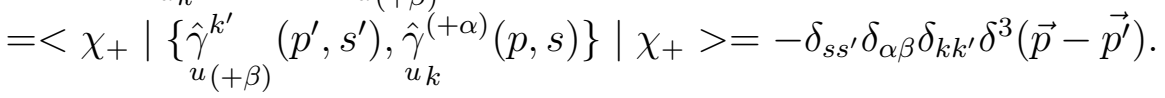

The expressions of causal Green's functions of distorted ordinary goyaks and their generalization can be readily written

$$
\begin{aligned}
& {\underset{u}{F}}_{F}^{\theta}\left(\theta_{+}-\theta_{-}\right)=-i<\chi_{-}\left|T \underset{u}{\hat{\Psi}}\left(\theta_{+}\right) \underset{u}{\overline{\hat{\Psi}}}\left(\theta_{-}\right)\right| \chi_{-}>_{0} /\left(u_{+} u_{-}\right)_{0}= \\
& =-i \int \frac{d^{3} p_{u}}{(2 \pi)^{3 / 2}}{\underset{u}{+p}}_{\left(\theta_{+k}\right)}^{\Psi_{u+p}}\left(\theta_{-k}\right) \theta\left(u_{+}^{0}-u_{-}^{0}\right)+i \int \frac{d^{3} p_{u}}{(2 \pi)^{3 / 2}} \overline{\bar{u}}_{-p}\left(\theta_{-k}\right) \underset{u_{-p}}{\Psi}\left(\theta_{+k}\right) \theta\left(u_{-}^{0}-u_{+}^{0}\right) ;
\end{aligned}
$$

and $\underset{u_{F}}{\widetilde{G}_{F}^{\theta}}\left(\theta_{+}-\theta_{-}\right)=\sum_{r^{\lambda}=1}^{N_{\lambda}}\left|\bar{c}\left(r^{\lambda}\right)\right|^{2}{\underset{u}{\lambda}}_{r^{\lambda}}^{r^{\lambda}}\left(\theta_{+}-\theta_{-}\right)$, provided $\underset{u_{\lambda}}{r^{r^{\lambda}}}\left(\theta_{+}-\theta_{-}\right)=-i T\left(\underset{u_{\lambda}}{r_{r^{\lambda}}}\left(\theta_{+}\right) \bar{\Psi}_{u_{\lambda}}^{r^{\lambda}}\left(\theta_{-}\right)\right)$,

where $\theta_{ \pm}\left(\eta_{ \pm}, u_{ \pm}\right)$. Geometry realization requirement now must be satisfied for each ordinary goyak in terms of

$$
G_{u_{F}}^{\theta}(0)={\underset{\eta}{F}}_{F}(0) \quad \text { or } \quad \widetilde{G}_{F}^{\theta}(0)=\widetilde{G}_{F}(0)
$$

They are valid in the case if following relations hold for each distorted ordinary goyak:

$$
\begin{aligned}
& \sum_{k} \underset{u+p}{\Psi}\left(\theta_{+k}(\eta, u)\right) \underset{u+p}{\Psi_{+p}}\left(\theta_{-k}(\eta, u)\right)= \\
& =\sum_{k} \underset{u+p}{\Psi^{\prime}}\left(\theta_{+k}^{\prime}(\eta, u)\right) \underset{\bar{\Psi}_{+p}^{\prime}}{\Psi_{+k}}\left(\theta_{-k}^{\prime}(\eta, u)\right)=\cdots=\underset{\eta_{+p}}{\Psi}(\eta) \underset{\eta_{+p}}{\bar{\Psi}}(\eta),
\end{aligned}
$$


and

$$
\begin{aligned}
& \sum_{k}{\underset{u}{\Psi}}_{-p}\left(\theta_{-k}(\eta, u)\right) \underset{u}{\Psi} \underset{-p}{\Psi}\left(\theta_{+k}(\eta, u)\right)= \\
& =\sum_{k} \bar{\Psi}_{u_{-p}^{\prime}}^{\bar{\Psi}_{-k}^{\prime}}\left(\theta^{\prime}(\eta, u)\right) \underset{u}{\Psi_{-p}^{\prime}}\left(\theta_{+k}^{\prime}(\eta, u)\right)=\cdots=\bar{\eta}_{-p}(\eta) \underset{\eta_{-p}}{\Psi}(\eta) .
\end{aligned}
$$

That is, distorted ordinary goyaks are being met in the special permissible combinations to realize the geometry. One final observation is worth recording. The following relations hold for the distortion transformation functions $f_{( \pm)}\left(\theta_{ \pm}\right)$:

$$
\begin{aligned}
& \sum_{k} f_{(+)}\left(\theta_{+k}\right) f_{(-)}\left(\theta_{-k}\right)=\sum_{k} f_{(+)}^{\prime}\left(\theta_{+k}^{\prime}\right) f_{(-)}^{\prime}\left(\theta_{-k}^{\prime}\right)=\cdots=i n v, \\
& \sum_{k} f_{(-)}\left(\theta_{-k}\right) f_{(+)}\left(\theta_{+k}\right)=\sum_{k} f_{(-)}^{\prime}\left(\theta_{-k}^{\prime}\right) f_{(+)}^{\prime}\left(\theta_{+k}^{\prime}\right)=\cdots=i n v .
\end{aligned}
$$

We have arrived at a quite promising and entirely satisfactory proposal to answer some of the original questions, namely those: what is the essential content of our notion of quarks and quantum number of color? What is the physical origin of them and especially the Color Confinement principle? To answer these queries below, in special case of local angles $\theta_{ \pm k}(\eta, u)$ with $(k)$ running from 1 to 3, we may think of the function $\underset{u}{\Psi}\left(\theta_{+k}(\eta, u)\right)$ at fixed $(k)$ as being $u$-component of bi-spinor field of "quark" $\hat{q}_{k}$, and of $\Psi_{u}^{\lambda}\left(\theta_{-k}(\eta, u)\right)$ an $u$-component of conjugate bi-spinor field of "anti-quark" $\overline{\hat{q}}_{k}$. Thus, the local rotations through the angles $\theta_{+k}(\eta, u)$ and $\theta_{-k}(\eta, u)$ yield the quarks and anti-quarks, respectively. The index $(k)$ refers to so-called "color" degrees of freedom in the case of rotations through the angles $\theta_{+k}(\eta, u)$ and "anti-color" degrees of freedom in the case of $\theta_{-k}(\eta, u)$. Here we leave the $\eta$-components of quark bi-spinor fields implicit, which are plane waves. Surely, one may readily perform a transition to conventional quark fields $\Psi_{f}\left(x_{f}\right)$ on $M^{4}$ (sec. 5.6). Thus, a quark is a fermion with the half-integral spin and certain color degree of freedom. As it was seen there are exactly three colors and the usual nomenclature is: $\hat{q}_{1}=$ red quark, $\hat{q}_{2}=$ green quark, $\hat{q}_{3}=$ blue quark. Due to eq.(15.8), one gets

$$
\begin{aligned}
& \sum_{k} \hat{q}_{k p} \overline{\hat{q}}_{k p}=\sum_{k}{\hat{q^{\prime}}}_{k p}{\overline{\hat{q^{\prime}}}}_{k p}=\cdots=i n v=\underset{\eta+p}{\Psi}(\eta) \bar{\eta}_{+p}(\eta), \\
& \sum_{k} \overline{\hat{q}}_{-k p} \hat{q}_{-k p}=\sum_{k}{\overline{\hat{q^{\prime}}}}_{-k p}{\hat{q^{\prime}}}_{-k p}=\cdots=i n v=\bar{\eta}_{-p}(\eta) \underset{\eta_{-p}}{\Psi}(\eta),
\end{aligned}
$$

which utilize the whole idea of Color(Quark) Confinement principle. According to it the quarks act in the special combinations of "color singlets" to realize the geometry.

Only two color singlets are available (see section 16.3)

$$
(q \bar{q})=\frac{1}{\sqrt{3}} \delta_{k k^{\prime}} \hat{q}_{k} \overline{\hat{q}}_{k^{\prime}}=i n v, \quad(q q q)=\frac{1}{\sqrt{6}} \varepsilon_{k l m} \hat{q}_{k} \hat{q}_{l} \hat{q}_{m}=i n v .
$$

If this picture of the color confinement turns out to reflect the actual situation in nature, quarks would be fields without free particles associated to them. Color would be confined, and the spectrum of hadrons (permissible combinations of quarks) would emerge as the spectrum of the color singlet states. Unwanted states (since not seen) like quarks $(q)$, (color triplets) or diquarks $(q q)$ (color sextets) are eliminated by construction at the very beginning. 


\section{Gauge Principle; Internal Symmetries}

As it was seen, the principle of identity holds for ordinary regular goyaks, according to it each goyak in the lowest state can be regarded as a result of transition into the state $\left(s_{0}\right)$ from an arbitrary state, in which the goyaks assumed to be distorted. This is succinctly stated below

$$
\begin{aligned}
& \underset{u_{\lambda}}{\Psi}=f_{(+)}^{-1}\left(\theta_{+k}\right) \underset{u_{\lambda}}{\Psi}\left(\theta_{+k}\right)=f_{(+)}^{-1}\left(\theta_{+l}^{\prime}\right) \underset{u_{\lambda}}{\Psi}\left(\theta_{+l}^{\prime}\right)=\cdots, \\
& \Psi_{u}^{\lambda}=\Psi_{u}^{\lambda}\left(\theta_{-k}\right) f_{(-)}^{-1}\left(\theta_{-k}\right)=\Psi_{u}^{\prime \lambda}\left(\theta_{-l}^{\prime}\right) f_{(-)}^{-1}\left(\theta_{-l}^{\prime}\right)=\cdots
\end{aligned}
$$

Hence the following transformations may be implemented on the distorted ordinary goyaks:

$$
\begin{aligned}
& \Psi_{u}^{\Psi^{\prime}}\left(\theta_{+l}^{\prime}\right)=f_{(+)}\left(\theta_{+l}^{\prime}\right) \underset{u}{\Psi}=f_{(+)}\left(\theta_{+l}^{\prime}\right) f_{(+)}^{-1}\left(\theta_{+k}\right) \underset{u_{\lambda}}{\Psi}\left(\theta_{+k}\right), \\
& \underset{u}{\Psi^{\prime \lambda}}\left(\theta_{-l}^{\prime}\right)=\underset{u}{\Psi^{\lambda}} f_{(-)}\left(\theta_{-l}^{\prime}\right)=\underset{u}{\Psi}\left(\theta_{-k}\right) f_{(-)}^{-1}\left(\theta_{-k}\right) f_{(-)}\left(\theta_{-l}^{\prime}\right) .
\end{aligned}
$$

The transformations take the standard form

$$
\begin{aligned}
& \underset{u_{\lambda}}{\Psi^{\prime}}\left(\theta_{+l}^{\prime}\right)=f_{l k}^{(+)} \underset{u_{\lambda}}{\Psi}\left(\theta_{+k}\right)=f\left(\theta_{+l}^{\prime}, \theta_{+k}\right) \underset{u_{\lambda}}{\Psi}\left(\theta_{+k}\right), \\
& \underset{u}{\Psi^{\prime \lambda}}\left(\theta_{-l}^{\prime}\right)=\underset{u}{\Psi^{\lambda}}\left(\theta_{-k}\right) f_{k l}^{(-)}=\underset{u}{\Psi^{\lambda}}\left(\theta_{-k}\right) f^{*}\left(\theta_{-l}^{\prime}, \theta_{-k}\right) \mid{ }_{\theta_{-l}^{\prime}}=\theta_{+l}^{\prime} \\
& \theta_{-k}=\theta_{+k}
\end{aligned}
$$

provided

$$
\begin{aligned}
& f_{l k}^{(+)}=\exp \left\{\chi_{l k}^{R}-i \chi_{l k}^{J}\right\}, \quad f_{k l}^{(-)}=\left(f_{l k}^{(+)}\right)^{*}, \\
& \chi_{l k}^{R}=\chi_{R}\left(\theta_{+l}^{\prime}\right)-\chi_{R}\left(\theta_{+k}\right), \quad \chi_{l k}^{J}=\chi_{J}\left(\theta_{+l}^{\prime}\right)-\chi_{J}\left(\theta_{+k}\right) .
\end{aligned}
$$

The transformation functions are regarded as the operators in the space of internal degrees of freedom labeled by $( \pm k)$, corresponding to distortion rotations around the axes $( \pm k)$ by the angles $\theta_{ \pm k}(\eta, u)$. We make proposition that local distortion rotations through the angles $\theta_{ \pm k}(\eta, u)$ with different $(k)$ are incompatible.

It is clear that because of incompatibility they can not be realized simultaneously. Hence

the operators $f_{l k}^{( \pm)}$with different $(l k)$ cannot have simultaneous eigen-states, i.e. measurements on their observables do not yield definite values (they "spread"). Transformation operators $f_{l k}^{( \pm)}$obey the commutation relations of incompatibility of distortion rotations

$$
\begin{aligned}
& f_{l k}^{(+)} f_{c d}^{(+)}-f_{l d}^{(+)} f_{c k}^{(+)}=\left\|f^{(+)}\right\| \varepsilon_{l c m} \varepsilon_{k d n} f_{n m}^{(-)}, \\
& f_{k l}^{(-)} f_{d c}^{(-)}-f_{d l}^{(-)} f_{k c}^{(-)}=\left\|f^{(-)}\right\| \varepsilon_{l c m} \varepsilon_{k d n} f_{m n}^{(+)},
\end{aligned}
$$

where $l, k, c, d, m, n=1,2,3$. As far as distorted ordinary goyaks have took participation in the realization of geometry $G(2.2 .3)$ instead of regular ones, the principle of identity of regular goyaks directly leads to the equivalent statement, which is known in physics under the name of Gauge Principle: an action integral of any physical system, defined in flat manifold $G(2.2 .3)$, must be invariant under arbitrary transformations eq.(16.3). This principle is valid for any physical system, which can be treated as a definite system of distorted ordinary goyaks with arbitrary internal degrees of freedom. In accordance with eq.(9.2) the total bi-spinor field of distorted goyak, which realizes the representation $D\left(\frac{1}{2}, 0\right) \otimes D\left(0, \frac{1}{2}\right) \otimes D\left(\frac{1}{2}, 0\right) \otimes D\left(0, \frac{1}{2}\right)$ of the group $\widehat{S O}(6.6)$, reads in component form $\Psi_{k}(\zeta)=\underset{\eta}{\Psi}(\eta) \underset{u_{\lambda}}{\underset{\Psi}{(}}\left(\theta_{+k}\right), \quad \bar{\Psi}_{k}(\zeta)=\underset{u_{\lambda}}{\Psi}\left(\theta_{+k}\right) \underset{\eta}{\Psi}(\eta)$, where $\underset{\eta}{\Psi}(\eta)$ is simply plane wave bispinor. Taking into account eq.(16.3), we get the equivalent transformations implemented 
on the total fields

$$
\Psi_{l}^{\prime}(\zeta)=f\left(\theta_{+l}^{\prime}(\zeta), \theta_{+k}(\zeta)\right) \Psi_{k}(\zeta), \quad \bar{\Psi}_{l}^{\prime}(\zeta)=\bar{\Psi}_{k}(\zeta) f^{*}\left(\theta_{-l}^{\prime}(\zeta), \theta_{-k}(\zeta)\right)
$$

They can be written succinctly

$$
\Psi^{\prime}(\zeta)=U(\theta(\zeta)) \Psi(\zeta), \quad \bar{\Psi}^{\prime}(\zeta)=\bar{\Psi}(\zeta) U^{+}(\theta(\zeta))
$$

Matrix notation is employed in eq.(16.7): $\Psi=\left\{\Psi_{k}\right\}, \quad U(\theta)=\left\{f\left(\theta_{+l}^{\prime}, \theta_{+k}\right)\right\}$. Particularly, in the case of local transformations through the angles $\theta_{ \pm k}(\zeta)$ with the color index $(k)$ running from 1 to 3 , the transformations eq.(16.7) yield the following ones implemented on the quark fields defined on manifold $G(2.2 .3)$ :

$$
\hat{q}_{l}^{\prime}(\zeta)=f\left(\theta_{+l}^{\prime}(\zeta), \theta_{+k}(\zeta)\right) \hat{q}_{k}(\zeta), \quad \overline{\hat{q}}_{l}^{\prime}(\zeta)=\overline{\hat{q}}_{k}(\zeta) f^{*}\left(\theta_{-l}^{\prime}(\zeta), \theta_{-k}(\zeta)\right)
$$

or in matrix notation

$$
\hat{q}^{\prime}(\zeta)=U(\theta(\zeta)) \hat{q}(\zeta), \quad \overline{q^{\prime}}(\zeta)=\overline{\hat{q}}(\zeta) U^{+}(\theta(\zeta)),
$$

where $\hat{q}(\zeta)=\left\{\hat{q}_{k}(\zeta)\right\}=\left\{\underset{\eta}{\Psi}(\eta) \hat{q}_{k}\right\}$. As we will see below, due to the incompatibility commutation relations (16.5), the transformation matrices $\{U\}$ generate the unitary group of internal symmetries $U(1), S U(2), S U(3)$. In order to utilize a gauge principle in its concrete expression, below we discuss different possible models.

\subsection{The Local Group $U^{l o c}(1)$ of Electromagnetic Interactions}

At the beginning we discuss the most simple case of one-dimensional local transformations, through the local angles $\theta_{+1}(\zeta)$ and $\theta_{-1}(\zeta)$

$$
f^{(+)}=\left(\begin{array}{lll}
f_{11}^{(+)} & 0 & 0 \\
0 & 1 & 0 \\
0 & 0 & 1
\end{array}\right), \quad f^{(-)}=\left(f^{(+)}\right)^{+} .
$$

The commutation relations (16.5) of incompatibility of distortion rotations reduced to identity $f_{11}^{(+)}=\left\|f^{(+)}\right\|$. In considered case $\chi_{R}\left(\theta_{+1}\right)=\chi_{R}\left(\theta_{-1}\right)$, and $f_{11}^{(+)}=f_{(+)}\left(\theta_{+1}\right) f_{(-)}\left(\theta_{-1}\right)=$ $f\left(\theta_{+1}, \theta_{-1}\right)$. Combining eq.(16.4) and eq.(16.9), it holds

$$
\Psi^{\prime}(\zeta)=U(\theta) \Psi(\zeta), \quad \bar{\Psi}^{\prime}(\zeta)=\bar{\Psi}(\zeta) U^{*}(\theta)
$$

provided by the transformation function

$$
f_{11}^{(+)}=U(\theta)=f\left(\theta_{+1}(\zeta), \theta_{-1}(\zeta)\right)=\exp \left\{-i \chi_{J}^{(+)}\left(\theta_{+1}\right)+i \chi_{J}^{(-)}\left(\theta_{-1}\right)\right\} .
$$

It may give rise to $U(\theta)=e^{-i \theta}$, where $\theta \equiv \chi_{J}^{(+)}\left(\theta_{+1}\right)-\chi_{J}^{(-)}\left(\theta_{-1}\right)$. The strength of interaction is specified by a single coupling $Q$, which is called electrical charge. A set of transformations generates a commutative Abelian unitary local group of electromagnetic interactions $U^{l o c}(1)$. That is, Lie group $G$ is realized as a $G=U^{l o c}(1)=S O^{l o c}(2)$, with one-dimensional trivial algebra $\hat{g}_{1}=R^{1}$. The invariance under the local group $U^{\text {loc }}(1)$ leads to electromagnetic field, the massless quanta of which - photons are electrically neutral, just because of the conditions (15.8) and eq.(16.12):

$$
f\left(\theta_{+1}, \theta_{-1}\right)=f\left(\theta_{+1}^{\prime}, \theta_{-1}^{\prime}\right)=\cdots \text { inv } .
$$




\subsection{Unitary Local Group $S U^{l o c}(2)$ of Weak Interactions}

Next we consider a particular case of two-dimensional local transformations through the angles $\theta_{ \pm m}(\zeta)$ around two axes $(m=1,2)$. The matrix function of transformation is written down

$$
f^{(+)}=\left(\begin{array}{lll}
f_{11}^{(+)} & f_{12}^{(+)} & 0 \\
f_{21}^{(+)} & f_{22}^{(+)} & 0 \\
0 & 0 & 1
\end{array}\right), \quad f^{(-)}=\left(f^{(+)}\right)^{+} .
$$

So, the commutation relations (16.5) of incompatibility of distortion rotations give rise to non-trivial conditions

$$
\begin{array}{ll}
f_{11}^{(+)}=\left\|f^{(+)}\right\|\left(f_{22}^{(+)}\right)^{*}, & f_{21}^{(+)}=-\left\|f^{(+)}\right\|\left(f_{12}^{(+)}\right)^{*}, \\
f_{12}^{(+)}=-\left\|f^{(+)}\right\|\left(f_{21}^{(+)}\right)^{*}, & f_{22}^{(+)}=\left\|f^{(+)}\right\|\left(f_{11}^{(+)}\right)^{*},
\end{array}
$$

Hence $\left\|f^{(+)}\right\|=1$. One can readily infer the matrix $U(\theta)$ of gauge transformations of collection of fundamental fields

$$
U=e^{-i \vec{T} \vec{\theta}}=\left(\begin{array}{ll}
f_{11}^{(+)} & f_{12}^{(+)} \\
f_{21}^{(+)} & f_{22}^{(+)}
\end{array}\right),
$$

where $T_{i} \quad(i=1,2,3)$ are the matrix representation of generators of the group $S U(2)$ : $T_{i}=\frac{1}{2} \sigma_{i}, \quad\left[T_{i}, T_{j}\right]=i \varepsilon_{i j k} T_{k}, \sigma_{i}$ are Pauli's matrices, $\vec{\theta}\left(\theta_{1}, \theta_{2}, \theta_{2}\right)$ are the transformation parameters of $S U(2)$, and due to eq.(16.15) $U^{+} U=I$. The fundamental fields will come in multiplets, which form a basis for representations of the isospin group $S U(2)$

$$
U(\theta)=\left[\cos \frac{\theta}{2}-\frac{i}{\theta} \sin \frac{\theta}{2}\left(\begin{array}{cc}
\theta_{3} & \theta_{1}-i \theta_{2} \\
\theta_{1}+i \theta_{2} & -\theta_{3}
\end{array}\right)\right],
$$

$(\theta=|\vec{\theta}|)$. We get easily

$$
\begin{aligned}
& \frac{\theta_{1}}{\theta}=\frac{e^{\chi_{12}^{R}} \sin \chi_{12}^{J}}{\sqrt{1-e^{2 \chi_{11}^{R}} \cos ^{2} \chi_{11}^{J}}}, \quad \frac{\theta_{2}}{\theta}=-\frac{e^{\chi_{12}^{R}} \cos \chi_{12}^{J}}{\sqrt{1-e^{2 \chi_{11}^{R}} \cos ^{2} \chi_{11}^{J}}}, \\
& \frac{\theta_{3}}{\theta}=\frac{e^{\chi_{11}^{R} \sin \chi_{11}^{J}}}{\sqrt{1-e^{2 \chi_{11}^{R}} \cos ^{2} \chi_{11}^{J}}}, \quad \theta=2 \arccos \left(e^{\chi_{11}^{R}} \cos \chi_{11}^{J}\right), \quad e^{\chi_{11}^{R}} \leq 1 .
\end{aligned}
$$

The following relations hold:

$$
\chi_{11}^{R}=\chi_{22}^{R}, \quad \chi_{12}^{R}=\chi_{21}^{R} \quad \chi_{11}^{J}+\chi_{22}^{J}=0, \quad \chi_{21}^{J}+\chi_{12}^{J}=\pi, \quad \chi_{12}^{R}=\frac{1}{2} \ln \left(1-e^{2 \chi_{11}^{R}}\right) .
$$

Hence, three functions $\chi_{11}^{R}, \chi_{11}^{J}$ and $\chi_{12}^{J}$ or the angles $\theta_{+1}^{\prime}, \theta_{+1}$ and $\theta_{+2}$ are parameters of the group $S U^{l o c}(2)$

$$
\chi_{11}^{R}=\chi_{R}\left(\theta_{+1}^{\prime}\right)-\chi_{R}\left(\theta_{+1}\right), \quad \chi_{11}^{J}=\chi_{J}\left(\theta_{+1}^{\prime}\right)-\chi_{J}\left(\theta_{+1}\right), \quad \chi_{12}^{J}=\chi_{J}\left(\theta_{+1}^{\prime}\right)-\chi_{J}\left(\theta_{+2}\right) .
$$

Therefore, the local gauge transformations of collection of fundamental fields read $\Psi^{\prime}(\zeta)=$ $U(\theta) \Psi(\zeta)$, where physical field $\Psi(\zeta)$ is a column vector. Continuing along this line, the Lagrangian must be invariant under local gauge transformations, as well by introducing non-Abelian vector gauge fields of weak interactions. 


\subsection{Unitary Local Group $S U^{l o c}(3)$ of Strong Interactions}

Finally we turn to the case, when gauge transformations around all three axes are local. Then, there are nine possible transformations with the functions $f_{l k}^{(+)}, \quad(l, k=1,2,3)$ :

$$
f^{(+)}=\left(\begin{array}{ccc}
f_{11}^{(+)} & f_{12}^{(+)} & f_{13}^{(+)} \\
f_{21}^{(+)} & f_{22}^{(+)} & f_{23}^{(+)} \\
f_{31}^{(+)} & f_{32}^{(+)} & f_{33}^{(+)}
\end{array}\right), \quad f^{(-)}=\left(f^{(+)}\right)^{+}
$$

Incompatibility commutation relations (16.5) yield the unitary condition $U^{-1}=U^{+}$, $f^{(+)} \equiv U$, and also $\|U\|=1$. Then $U(\theta)=e^{-\frac{i}{2} \vec{\lambda} \vec{\theta}}$, where $\frac{\lambda_{i}}{2} \quad(i=1, \ldots, 8)$ are the matrix representation of generators of the group $S U(3):\left[\frac{\lambda_{i}}{2}, \frac{\lambda_{k}}{2}\right]=i f_{i k l} \frac{\lambda_{l}}{2}$, the antisymmetric structure constants are denoted by $f_{i k l}$. The transformations are implemented on the column vector fundamental quark fields $\hat{q}^{\prime}=U(\theta) \hat{q}$. Right through differentiation one infers at once $\vec{\lambda} \overrightarrow{d \theta}=2 i U^{+} d U$, or

$$
\vec{\theta}=-\int \operatorname{Im}\left(\operatorname{tr}\left(\vec{\lambda}\left(f^{(-)} d f^{(+)}\right)\right)\right)
$$

provided $\operatorname{Re}\left(\operatorname{tr}\left(\vec{\lambda}\left(f^{(-)} d f^{(+)}\right)\right)\right) \equiv 0$. At the infinitesimal transformations $\theta_{i} \ll 1$

$$
\left(\begin{array}{l}
q_{1}^{\prime} \\
q_{2}^{\prime} \\
q_{3}^{\prime}
\end{array}\right)=\left\{1-\frac{i}{2}\left(\begin{array}{ccc}
\theta_{3}+\frac{1}{\sqrt{3}} \theta_{8} & \theta_{1}-i \theta_{2} & \theta_{4}-i \theta_{5} \\
\theta_{1}+i \theta_{2} & -\theta_{3}+\frac{1}{\sqrt{3}} \theta_{8} & \theta_{6}-i \theta_{7} \\
\theta_{4}+i \theta_{5} & \theta_{6}+i \theta_{7} & -\frac{2}{\sqrt{3}} \theta_{8}
\end{array}\right)\right\} \cdot\left(\begin{array}{l}
q_{1} \\
q_{2} \\
q_{3}
\end{array}\right)
$$

we get

$$
\begin{aligned}
& \theta_{1} \approx 2 e^{\chi_{12}^{R}} \sin \chi_{12}^{J}, \quad \theta_{3} \approx \sin \chi_{33}^{J}+2 \sin \chi_{11}^{J}, \quad \theta_{5} \approx 2\left(1-e^{\chi_{13}^{R}} \cos \chi_{13}^{J}\right), \\
& \theta_{2} \approx 2\left(1-e^{\chi_{12}^{R}} \cos \chi_{12}^{J}\right), \quad \theta_{4} \approx 2 e^{\chi_{13}^{R}} \sin \chi_{13}^{J}, \quad \theta_{6} \approx 2 e^{\chi_{23}^{R}} \sin \chi_{23}^{J} \text {, } \\
& \theta_{7} \approx 2\left(1-e^{\chi_{23}^{R}} \cos \chi_{23}^{J}\right), \quad \theta_{8} \approx-\sqrt{3} \sin \chi_{33}^{J},
\end{aligned}
$$

provided

$$
\chi_{l l}^{R} \approx 0, \quad \chi_{l k}^{R} \approx \chi_{k l}^{R}, \quad \chi_{l k}^{J} \approx \chi_{k l}^{J}, \quad(l \neq k) \quad \sin \chi_{11}^{J}+\sin \chi_{22}^{J}+\sin \chi_{33}^{J} \approx 0 .
$$

As it was seen in section 15 , the existence of the internal symmetry group $S U_{C}^{\text {loc }}(3)$ allows oneself to introduce a gauge theory in color space, with the color charges as exactly conserved quantities. The local color transformations are implemented on the colored quarks right through a $S U_{C}^{\text {loc }}(3)$ rotation matrix $U$ eq.(16.21) in the fundamental representation.

\section{Discussion and Conclusions}

At this point we cut short our exposition of the theory and reflect upon the results far obtained. A number of conclusions may be drawn and the main features of suggested theory are outlined below. We have finally arrived at an entirely satisfactory proposal to 
answer all original questions posed in introduction. The following scenario is cleared up, the whole idea of which comes to this: the basic concepts of geometry, fundamental fields with various quantum numbers, internal symmetries and so forth; also the basic principles of Relativity, Quantum, Gauge and Color Confinement, are all derivative. They come into being simultaneously.

We have mainly developed the mathematical framework for our viewpoint. We recognize that this kind of mathematical treatment has been necessarily introductory by nature, hence our discussion has been rather general and abstract. The numerous issues in suggested theory still remain to be resolved. Surely the more realistic complete theory is a subject for further research.

We start with a quite promising proposal of generating the group of gravitation by hidden local internal symmetries. Meanwhile, under the reflection of shadow fields from Minkowski flat space to Riemannian, one framed the idea of general gauge principle of local internal symmetries $\mathrm{G}$ into requirement of invariance of physical system of reflected fields on $R^{4}$ under the Lie group of gravitation $G_{R}$ of local gauge transformations generated by $\mathrm{G}$. This yields the invariance under wider group of arbitrary curvilinear coordinate transformations in $R^{4}$. While the energy -momentum conservation laws are well -defined. The fascinating prospect emerged for resolving or mitigating a shortage of controversial problems of gravitation including its quantization by exploiting whole advantages of field theory in terms of flat space. In the aftermath, one may carry out an inverse reflection into $R^{4}$ whenever it will be needed. It was proved that only gravitational attraction exists. One may easily infer Einstein's equation of gravitation, but with strong difference at the vital point of well -defined energy -momentum tensor of gravitational field and conservation laws. Nevertheless, for our part we prefer standard gauge invariant Lagrangian eq.(4.11), while the functions eq.(2.3) ought to be defined. We considered the gravitational interaction with hidden Abelian group $G=U^{l o c}(1)$ with the base $G(2.3)$ of principle bundle as well as the general distortion of manifold $G(2.2 .3)$, which yields both the curvature and inner-distortion of space and time. Regarding the persistent processes of creation and annihilation of regular goyaks in the lowest state, we develop the formalism of operator manifold $\hat{G}(2.2 .3)$, which is the mathematical foundation for our viewpoint in the second and third parts. This is a guiding formalism of our approach and a still wider generalization of familiar methods of secondary quantization with appropriate expansion over the processes involving geometric objects. This generalization yields the quantization of geometry, which differs in principle from all earlier suggested schemes. One readily found out a contingency arisen at the very beginning that all states of goyak are degenerate with a degree of degeneracy equal 2. That is, along many quantum numbers of definite state of goyak there is half-integral spin number. Thus, the goyaks are turned out to be fermions with the half-integral spins. As it was seen in later sections, this gives rise to the spins of particles. The nature of $\hat{G}(2.2 .3)$ provides its elements with both the field and geometric aspects. In this context it comes out that the geometry is derivative by nature. It may be realized in the special system of regular goyaks if only some subsidiary condition holds for each participating ordinary goyak. In the sequel, the manifold $G(2.2 .3)$ gives rise to the Minkowski flat space $M^{4}$. By this we have arrived at an answer to the question of the physical origin of the space-time and the Principle of Relativity. We have briefly treated the quantum theory situation corresponding to simultaneous presence of many identical goyak fields. In last part we extend the scopes of treatment by considering the processes involving distorted goyaks. For the second choice 
of realization of geometry we have following recipe: it is admitted that distorted ordinary goyaks have took participation in the realization of geometry instead of regular ordinary goyaks. It comes out that distorted goyaks are acting in special permissible combinations to realize the geometry. The wave functions and conjugate functions of distorted goyaks enable one to introduce the bi-spinor (conjugate) field of quarks and anti-quarks as the operator fields in the color space of internal degrees of freedom, corresponding to local distortion transformations around axes $k=1,2,3$. We have assumed that local distortion rotations around the different axes are incompatible, i.e. they cannot occur simultaneously. It has been shown that quarks are being met in some special combinations to emerge in geometry only in color singlets. That is, they obey exact Color Confinement principle and the spectrum of hadrons would emerge as the spectrum of the color singlet states. As it was seen, the identity principle holds for ordinary regular goyaks, according to it each goyak in the lowest state may be regarded as the result of transformation from arbitrary higher state, in which the goyaks are assumed to be distorted. It is impressive to learn that this principle, underlies the most important gauge principle. A global charge conservation corresponds to global gauge invariance. But a requirement of expansion of Lie group from global to local symmetry can be satisfied by introducing the gauge fields of interactions. This invariance in its concrete expressions yields the different models of possible interactions: one-dimensional transformations provide a local internal symmetry of electromagnetic interactions, with an Abelian group $U^{l o c}(1)$ : two-dimensional transformations lead to local internal symmetry of weak interactions, with the local non-Abelian unitary group $S U^{l o c}(2)$; and finally, three-dimensional transformations yield the strong interactions, with the local group $S U_{C}^{\text {loc }}(3)$.

All these results assure us that the more realistic final theory of particles and interactions can be found within the context of the theory of goyaks. We believe we have made good headway by presenting a reasonable framework whereby one will be able to verify the basic ideas of suggested theory.

\section{Acknowledgements}

This work was supported in part by the International Atomic Energy Agency and the United Nations Educational, Scientific and Cultural Organization. It is a pleasure to thank for their hospitality the International Centre for Theoretical Physics, Trieste, where part of this work was done, and its director Professor Abdus Salam. I express my gratitude to colleagues for fruitful discussions and useful comments on the various issues treated in this paper, among them Professor V.A.Ambartsumian, Professor J.Strathdee and also all participants of the seminars. I am greatly indebted to A.M.Vardanian and K.L.Yerknapetian for their steady support.

\section{Appendix}

\section{The Wave Equation of Distorted Goyak}

As a Fermi field defined in distorted manifold $\underset{u}{G}(23)$, the bi-spinor field of distorted ordinary goyak is described by invariant Lagrangian, which, according to tetrad formalism 
[13] can be written [1-5]:

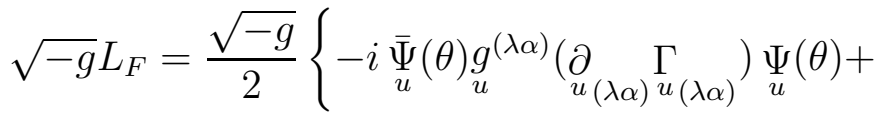

$$
\begin{aligned}
& \left.+i \underset{u}{\bar{\Psi}}(\theta)\left(\overleftarrow{u}_{u(\lambda \alpha)}^{\overleftarrow{\partial}}-\bar{\Gamma}_{u(\lambda \alpha)}\right) \underset{u}{g(\lambda \alpha)} \underset{u}{\Psi}(\theta)+2 m \underset{u}{\bar{\Psi}}(\theta) \underset{u}{\Psi}(\theta)\right\}
\end{aligned}
$$

where $\underset{u}{g^{(\lambda \alpha)}}(\theta)$ are matrix realization of basis $\underset{u}{e^{(\lambda \alpha)}}(\theta)$ :

$$
\underset{u}{g^{(\lambda \alpha)}}(\theta)=\underset{u}{V_{(\tau \beta)}^{(\lambda \alpha)}}(\theta) \gamma_{0}^{(\tau \beta)}
$$

The matrix functions $\Gamma_{u_{(\lambda \alpha)}}(\theta)$ and $\bar{\Gamma}_{u_{(\lambda \alpha)}}(\theta)$ in terms of Ricci rotation coefficients read

$$
\Gamma_{u(\lambda \alpha)}(\theta)=\frac{1}{4} \Delta_{(\lambda \alpha)(i, l)(m, p)} \gamma_{0}^{(i, l)} \gamma_{0}^{(m, p)}, \quad \bar{\Gamma}_{u(\lambda \alpha)}(\theta)=\frac{1}{4} \Delta_{(\lambda \alpha)(i, l)(m, p)} \gamma_{0}^{(m, p)} \gamma_{0}^{(i, l)}
$$

provided $\Delta_{(\lambda \alpha)(i, l)(m, p)}=\Delta_{(\lambda \alpha)(\tau \beta)(\rho \gamma)} V_{(i, l)}^{(\tau \beta)} V_{(m, p)}^{(\rho \gamma)}$, and

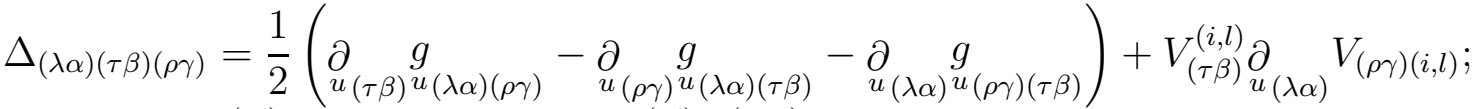

$$
\begin{aligned}
& g_{u_{(\lambda \alpha)(\tau \beta)}}=V_{(\lambda \alpha)}^{(i, l)} V_{(i, l)(\tau \beta)}=g_{(i, l)(m, p)} V_{(\lambda \alpha)}^{(i, l)} V_{(\tau \beta)}^{(m, p)},
\end{aligned}
$$

$V_{(i, l)}^{(\lambda \alpha)}(\theta)$ are congruence parameters of curves (Latin indices refer to tetrad components). Here, as usual, we let first subscript in the parentheses labels the pseudo-vector components, when second refers to ordinary-vector components. At fixed metric the current ${\underset{u}{J}}^{(\lambda \alpha)}=\bar{u} \underset{u}{\Psi}(\theta) \underset{u}{g^{(\lambda \alpha)}}(\theta) \underset{u}{\Psi}(\theta)$ is conserved $\frac{1}{\sqrt{-g}}{ }_{u(\lambda \alpha)}\left(\sqrt{-g} \underset{u}{J^{(\lambda \alpha)}}\right)=0$. Field equations can be derived at once right through eq.(A.1) and variational principle of least action

$$
\begin{aligned}
& \left.\left[\underset{u}{i g^{(\lambda \alpha)}}(\theta) \underset{u_{(\lambda \alpha)}}{\partial_{u}}-\Gamma_{u(\lambda \alpha)}(\theta)\right)-m\right] \underset{u}{\Psi}(\theta)=0, \\
& \bar{\Psi}(\theta)\left[i\left(\overleftarrow{\partial}_{u(\lambda \alpha)}-\bar{\Gamma}_{u(\lambda \alpha)}(\theta)\right) g_{u}{ }^{(\lambda \alpha)}(\theta)-m\right]=0
\end{aligned}
$$

\section{References}

[1] Ter-Kazarian G.T., Selected Questions of Theoretical and Mathematical Physics, 1986, VINITI, N5322-B86, Moscow.

[2] Ter-Kazarian G.T.,Comm. Byurakan Obs., 1989,v.62, 1.

[3] Ter-Kazarian G.T., Astrophys. and Space Sci., 1992, v.194, 1.

[4] Ter-Kazarian G.T.,IC/94/290, ICTP Preprint, Trieste, Italy, 1, 1994.

[5] Ter-Kazarian G.T., hep-th/9510110, 1995.

[6] Weil H., Sitzungsber. d. Berl. Akad., 1918, 465. 
[7] Yang C.N., Mills R.L., Phys. Rev., 1954, v. 96, 191.

[8] Utiyama R., Phys. Rev., 1956, v.101, 1597.

[9] Dubrovin B.A., Novikov S.P., Fomenko A.T., Contemporary Geometry, 1986, Nauka, Moscow.

[10] Pontryagin L.S., Continous Groups, 1984, Nauka, Moscow.

[11] Weinberg S., 1972, Gravitation and Cosmology, J.W. and Sons, New York.

[12] Dirac P.A.M., 1975, General Theory of Relativity, A Wiley-Interscience Publ., New York.

[13] Fok V.A., Zeitsch. fur Phys., 1929, v.57, 261.

[14] Weinberg S., 1964, Phys. Rev. b133, 1318.

[15] Di Bartini, Dokl. Akad. Nauk, SSSR, v.163,4, 1965.

[16] Bjorken J.D., Drell S.D., Relativistic Quantum Fields, Mc Graw-Hill, New York, 1964. 